\title{
Three Essays on Corporate Governance in China
}

by

Zonghao Chen (Jeffrey)

A thesis submitted to the Victoria University of Wellington in fulfillment of the requirements for the degree of Doctor of Philosophy in Finance.

Victoria University of Wellington

2019 


\section{Abstract}

This thesis consists of three empirical papers on corporate governance in Chinese listed firms. The first essay examines the influence of director characteristics and ownership structure on director compensation. Over the period 2005 through 2015, we find that director compensation in Chinese listed firms is influenced by both director characteristics and ownership structure. We measure director compensation by both the propensity to be paid and the level of compensation. For independent directors, we find that director busyness, tenure, and ownership concentration positively influence and state-ownership negatively influences director compensation. For non-independent directors, we find that tenure positively influences and that both state-ownership and related directors negatively influence director compensation. Lastly, our evidence suggests that women directors in China are not underpaid.

The second essay examines the influence of rookie independent directors on board functions and firm performance in Chinese public companies from 2008 to 2014. We find that rookie independent directors attend more board meetings than seasoned independent directors. Independent directors with higher board meeting attendance are more likely to remain in the firm in the following year (lower turnover rate). This influence of board attendance on re-appointment is stronger for rookie independent directors. Further, we find that boards with more rookie independent directors tunnel less to controlling shareholders, suggesting that rookie independent directors are efficient monitors. Lastly, we find that firms with more rookie independent directors are associated with higher accounting returns. 
In the third essay, we investigate the influence of board networks on directors' career outcomes in Chinese public firms from 2005 to 2014. We find that board connections increase compensation for independent directors. We find that board connections are positively associated with director turnover for non-related directors, but negatively associated with director turnover for related directors. Further, we find that board connections lead to additional future directorships. Overall, we find that board connections both directly lead to higher compensation and indirectly through labor mobility and additional board seats. 


\section{Contents}

1 Board of director compensation in China: To pay or not to pay? How $\begin{array}{ll}\text { much to pay? } & 8\end{array}$

1.1 Introduction . . . . . . . . . . . . . . . . . . . . 9

1.2 Institutional background . . . . . . . . . . . . . . . 13

1.2.1 Ownership structure in China . . . . . . . . . . . . . . 13

1.2.2 Board structure in China . . . . . . . . . . . . . . . . . . 14

1.2.3 The independent director in China . . . . . . . . . . . . 15

1.3 Literature and hypothesis development . . . . . . . . . . . . . 16

1.3.1 Director compensation and director characteristics . . . . . . . . 16

1.3.2 Compensation and ownership structure . . . . . . . . . 18

1.4 Data and variable construction . . . . . . . . . . . . . . . . 19

1.4.1 Sample construction . . . . . . . . . . . . . . . 19

1.4.2 Dependent variables . . . . . . . . . . . . . . 20

1.4.3 Variables of interest . . . . . . . . . . . . . 20

1.4.4 Control variables . . . . . . . . . . . . . . . . . 21

1.5 Testing approach and results . . . . . . . . . . . . . 22

1.5.1 Testing approach . . . . . . . . . . . . . . . 22

1.5.2 Director compensation and characteristics . . . . . . . . 23

1.5.3 Director compensation and ownership structure . . . . . . . . 25

1.6 Robustness tests f . . . . . . . . . . . . . . . . 27

1.6.1 Zero pay and low pay . . . . . . . . . . . . . 27 
1.6.2 Tenure and new director . . . . . . . . . . . . . . . . . 28

1.6.3 Political connections and compensation . . . . . . . . . . . . 29

1.6.4 Time invariant director heterogeneity . . . . . . . . . . . . . 30

1.6.5 Ownership concentration and compensation (state-owned versus non-state-owned $\ldots \ldots \ldots 30$

1.7 Conclusion . . . . . . . . . . . . . . . . . . . . . . . 31

1.8 Tables . . . . . . . . . . . . . . . . . . . . . . . . . . 37

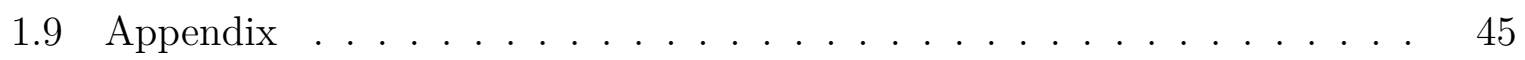

2 Rookie directors and firm performance: Evidence from China 48

2.1 Introduction . . . . . . . . . . . . . . . . . 49

2.2 Literature and hypothesis development . . . . . . . . . . . . . 52

2.3 Sample and variable construction . . . . . . . . . . . . . . 54

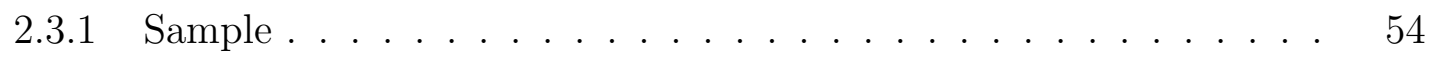

2.3.2 Dependent variables . . . . . . . . . . . . . . . . 55

2.3.3 Variables of interest . . . . . . . . . . . . . . 57

2.3.4 Control variables . . . . . . . . . . . . . . 57

2.4 Test approach and results . . . . . . . . . . . . . . . 58

2.4.1 Are rookie independent directors more diligent directors? . . . . . 58

2.4.2 Rookie directors and firm performance . . . . . . . . . . 60

2.4.3 What are the potential channels through which rookie independent directors affect firm performance? . . . . . . . . . . 61

2.4.4 Are rookie independent directors rewarded more for their efforts? 62

2.4.5 What kind of firms benefit more from rookie independent directors? 64

2.5 Discussion and Robustness Tests . . . . . . . . . . . . 65

2.5.1 Rookie independent directors and director busyness . . . . . . . 65

2.5.2 Endogeneity tests on firm performance . . . . . . . . . . . . 66

2.5.3 Alternative measure of ROS and ROA . . . . . . . . . . . 68 
2.5.4 Last-year director . . . . . . . . . . . . . . . . . 68

2.6 Conclusion . . . . . . . . . . . . . . . . . . . . . . . 68

2.7 Tables . . . . . . . . . . . . . . . . . . . . . . . 72

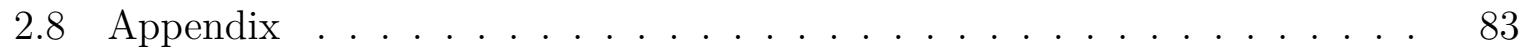

3 Board of director compensation in China: It pays to be connected 86

3.1 Introduction . . . . . . . . . . . . . . . . . . . 87

3.2 Literature and hypothesis development . . . . . . . . . . . . . . . 89

3.3 Sample and variable construction . . . . . . . . . . . . . . . . . . 91

3.3 .1 Sample . . . . . . . . . . . . . . . . . . . 91

3.3.2 Dependent variables . . . . . . . . . . . . . . . . . . . . . . . 92

3.3.3 Network prominence measure . . . . . . . . . . . . . . . . . . . . 93

3.3.4 Control variables . . . . . . . . . . . . . . . . . . 94

3.4 Testing approach and results . . . . . . . . . . . . . 95

3.4.1 Director network and directorship level compensation . . . . . . . 95

3.4 .2 Director network and director turnover . . . . . . . . . . . . . 97

3.4.3 Director network and further directorship . . . . . . . . . . . . . 98

3.4.4 Director network on total director compensation through labor mobility . . . . . . . . . . . . . . . . . . . . 98

3.5 Robustness . . . . . . . . . . . . . . . . . . . . 100

3.5.1 Related director . . . . . . . . . . . . . . . . . . 100

3.6 Conclusion . . . . . . . . . . . . . . . . . . . . . . . 101

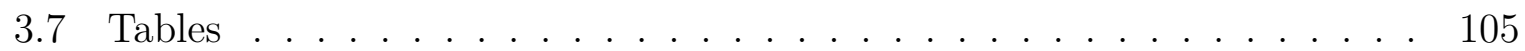

3.8 Appendix . . . . . . . . . . . . . . . . . . . . . . . . . . . . . . . . 121 


\section{List of Tables}

1.1 Summary statistics . . . . . . . . . . . . . . . . . . . 37

1.2 Cross correlations . . . . . . . . . . . . . . . . . . . . . . 38

1.3 Testing hypothesis 1 and $2 \ldots \ldots \ldots$. . . . . . . . . . . . . . . . . .

1.4 Estimated economic impact . . . . . . . . . . . . . . . . . 40

1.5 Robustness: Tenure and new director . . . . . . . . . . . . . . . . . 41

1.6 Robustness: Political connections . . . . . . . . . . . . . . . . . . . . 42

1.7 Robustness: Director effects . . . . . . . . . . . . . . . . . 43

1.8 Robustness: Ownership concentration (state-owned versus non-state-owned) 44

1.9 Variable definitions . . . . . . . . . . . . . . . . . 45

1.9 Variable definitions . . . . . . . . . . . . . . 46

1.9 Variable definitions . . . . . . . . . . . . . . . . . . 47

2.1 Summary statistics . . . . . . . . . . . . . . . . . . . 72

2.2 Cross correlations . . . . . . . . . . . . . . . . . 73

2.3 Rookie directors and board meeting attendance . . . . . . . . . . . . . 74

2.4 Rookie directors and firm performance . . . . . . . . . . . . . 75

2.5 Rookie directors and tunneling . . . . . . . . . . . . . 76

2.6 Rookie directors and director turnover f . . . . . . . . . . . . . . . . . 77

2.7 Rookie directors and firm performance (state-owned vs non-state-owned) 78

2.8 Robustness check: Rookie director appointments . . . . . . . . . . . . 79

2.9 Robustness check: The instrument variables 2SLS regressions . . . . . . . 80

2.10 Robustness check: Alternative measure of ROS and ROA . . . . . . . . . 81 
2.11 Robustness check: Last-year director and board meeting attendance . . . 82

2.12 Variable definitions . . . . . . . . . . . . . . . . . . 83

2.12 Variable definitions . . . . . . . . . . . . . . . . . 84

2.12 Variable definitions . . . . . . . . . . . . . . . . . 85

3.1 Board of directors with most network prominence each year . . . . . . 108

3.2 Summary statistics . . . . . . . . . . . . . . . . . . . . . 109

3.3 Cross correlations of network prominence and board of director compensation110

3.4 Network prominence and director compensation . . . . . . . . . . . . 111

3.5 Network prominence and director compensation (firm and director fixed effects) . . . . . . . . . . . . . . . . . . 112

3.6 Network prominence and director turnover . . . . . . . . . . . . . . . 113

3.7 Network prominence and director turnover (firm and director fixed effects) 114

3.8 Network prominence and directors' future directorship . . . . . . . . . . 115

3.9 Network prominence and directors' future directorship (director fixed effects)116

3.10 Indirect effect of network prominence on total compensation through turnover 117

3.11 Indirect effect of network prominence through related director on nonexecutive director compensation . . . . . . . . . . . . . . . . . . 118

3.12 Indirect effect of network prominence through related director on nonindependent director turnover . . . . . . . . . . . . . . . . . . . . 119

3.13 Indirect effect of network prominence through related director on nonindependent directors' future directorships . . . . . . . . . . . . . . . 120

3.14 Variable definitions . . . . . . . . . . . . . . . . . . . . 121

3.14 Variable definitions . . . . . . . . . . . . . . . . . . 122

3.14 Variable definitions . . . . . . . . . . . . . . . . . . . 123 
Chapter 1

Board of director compensation in

China: To pay or not to pay? How much to pay? 


\subsection{Introduction}

Ideally, a board of directors lessens agency problems between managers and shareholders. An important function of the board of directors is to monitor management (Jensen, 1986, Shleifer and Vishny, 1997, Hermalin and Weisbach, 2003). In addition, directors provide management guidance on the operation of the firm (Jensen, 1993, Adams and Ferreira, 2007, Adams, Hermalin, and Weisbach, 2010). Director compensation should motivate directors to perform these functions effectively (Adams, Hermalin, and Weisbach, 2010). Although director compensation is widely studied in developed countries, there is limited research on director compensation in China, where the ownership structure and governance issues differ from those in US and UK (Jiang and Kim, 2015). This study examines director compensation in China.

In the US and UK, ownership of listed firms is dispersed and the main conflict is between the managers and shareholders. Ideally, a director compensation scheme eases the agency problem between managers and shareholders. In contrast, in China, the ownership of listed firms is highly concentrated and the main agency conflict is between large and minority shareholders. Director compensation in China should be motivated to mitigate the conflict between large shareholders and minority shareholders. In practice, the compensation committee proposes the compensation scheme which is voted by the board of directors. With the concentrated ownership, the large shareholders in China have substantial influence over director compensation. Therefore, director compensation in China may not resolve conflicts between large and minority shareholders but simply reflect the influence of large shareholders.

Unlike previous literature focusing only on directors who are paid, our study includes directors who are ostensibly unpaid by the listed firms. The practice of ostensibly paying zero compensation is common in China, where $36 \%$ of non-independent directors and $6 \%$ of independent directors receive zero compensation. The scope of our study examines both the propensity of a director to be unpaid and the level of director compensation provides a complete picture of director compensation in China.

We investigate the influence of director characteristics and ownership structure on director compensation. Relative to the relationship between director characteristics and compensation, we explore the influences of gender, director busyness and director tenure on director compensation. Relative to the relationship between ownership structure and compensation, we explore the influence of state ownership, ownership concentration, excess control rights and connections to controlling shareholders on director compensation.

Female directors comprise $15 \%$ of the independent directors and $11 \%$ of non-independent directors in our sample. We find no evidence that in China female directors are under- 
paid. Our results are inconsistent with the literature that suggests women executives are underpaid (Fagenson and Jackson, 1993, Zelechowski and Bilimoria, 2004, Chen, Ezzamel, and Cai, 2011). However, our results support the literature that the underpayment of female executives declines in a multivariable setting with controls (Bertrand and Hallock, 2001). We find some evidence that non-independent women directors are less likely to be unpaid. To the best of our knowledge, this is the first work that investigates the influence of gender in Chinese boardrooms on both the propensity to be unpaid and the level of compensation.

In our sample, $31 \%$ of independent directors are busy directors (hold more than two directorships at the same time) and hold on average 2.14 directorships. We find that in China busy independent directors are less likely to be unpaid and receive a higher level of compensation. Specifically, for an independent director that holds more than two directorships, the probability of being unpaid decreases by $11.18 \%$ and the level of compensation increases by $2.78 \%{ }^{1}$ A strand of the literature advances that busy directors are of high quality (Fama and Jensen, 1983, Gilson, 1990, Kaplan and Reishus, 1990, Brickley, Linck, and Coles, 1999, Coles and Hoi, 2003, Brown and Maloney, 1999, Fich and Shivdasani, 2007, Field, Lowry, and Mkrtchyan, 2013). However, another strand of the empirical literature finds that the relationship between busy directors and compensation is mixed (Andreas, Rapp, and Wolff, 2012, Ertugrul and Hegde, 2008). Therefore, our study provides further evidence that director compensation increases with busyness. To our knowledge, this is the first work that examines the influence of busyness on director compensation in China.

The average tenure in our sample equals to 6.11 years for independent directors and 5.9 years for non-independent directors. We find that in China both independent directors and non-independent directors with longer tenure are less likely to be unpaid and receive a higher level of compensation. Specifically, a one standard deviation increase in tenure for an independent director implies that probability of being unpaid decreases by $13.53 \%$ and the level of compensation increases by $1.62 \% .^{2}$ Dou, Sahgal, and Zhang (2015) suggest that the directors with more experiences (measured by tenure) are better monitors and advisors. If the tenure of director serves as a good proxy for the quality of director, then the director with longer tenure should be less likely to be unpaid and receive a

\footnotetext{
${ }^{1}$ Assume $\operatorname{Prob}\left(\operatorname{Unpaid}\left(\right.\right.$ Busy director $\left.\left.(0 / 1)_{t}=0\right)\right)=\mathrm{A}$ and $\operatorname{Prob}\left(\operatorname{Unpaid}\left(\right.\right.$ Busy director $\left.\left.(0 / 1)_{t}=1\right)\right)=\mathrm{B}$, we calculate the percentage change in the probability being unpaid as

$$
\% \Delta\left(\operatorname{Unpaid}(0 / 1)_{t}\right)=\frac{B-A}{A}
$$

${ }^{2}$ Similarly, a one standard deviation increase in tenure for a non-independent director implies that probability of being unpaid decreases by $11.68 \%$ and the level of compensation increases by $13.94 \%$.
} 
higher level of compensation. In contrast, Chen, Ezzamel, and Cai (2011) find that in China the compensation of top three executives is negatively related to their tenure. Our study provides further evidence that director compensation increases with tenure and thus supports the idea that director tenure is an indicator of director quality.

The literature on director compensation and ownership structure is limited. ${ }^{3}$ We investigate how ownership structure affects the director compensation in China. China is an excellent laboratory to study this question alone to different ownership structures including state ownership, ownership concentration, excess control rights and connections to controlling shareholders.

State ownership is very common in China. In our sample, $48 \%$ of the listed firms are state-owned. We find that in China both independent and non-independent directors working in a state-owned company are more likely to be unpaid and receive a lower level of compensation. Specifically, for an independent director working in a state-owned company, the level of compensation decreases by $7.92 \%$. For a non-independent director working in a state-owned company, the probability of being unpaid increases by $31.20 \%$ and the level of compensation decreases by $11.57 \%$. Prior research finds that in China the state ownership reduces the level of CEO compensation (Firth, Fung, and Rui, 2007, Liang, Renneboog, and Sun, 2015). Moreover, Barontini and Bozzi (2011) find that in Italy the state ownership reduces the level of director compensation. Our findings provide further evidence that state ownership reduces director compensation.

The ownership of listed firms in China is concentrated. The ultimate controlling shareholders on average own $33 \%$ of the share of the listed firms. ${ }^{45}$ We find that in China independent directors are less likely and non-independent directors are more likely to be unpaid when the ultimate controlling shareholders have more ownership in the listed firms. The level of compensation for both independent directors and non-independent directors increases with the ownership of the ultimate controlling shareholders. Specifically,

\footnotetext{
${ }^{3}$ There are a few of studies concerning the relationship between ownership structure and director compensation in other countries. For example, Barontini and Bozzi (2011) study how ownership structure affects the director compensation in Italian listed firms. Pinto and Leal (2013) study how ownership structure influences the board compensation in Brazilian listed companies. Munisi and Mersland (2016) investigate how ownership composition affects the board compensation in listed Sub-Saharan African companies.

${ }^{4}$ In China, the proportion of ownership measures the proportion of cash-flow rights since dual class shares structure is not allowed.

${ }^{5}$ We use the ownership of ultimate controlling shareholder rather than the ownership of the largest shareholder to measure the ownership concentration. In China, pyramid ownership structure is very common. The ultimate controlling shareholders may control the listed firms through one of their subsidiaries. In this way, the ownership of the largest shareholders in the listed firms may exaggerate the economic stake of the actual controllers in the listed firms. For example, company A owns $51 \%$ of company B and company B owns $51 \%$ of company $\mathrm{C}$ (the listed firm). The largest shareholder of company $\mathrm{C}$ (the listed firm) is firm B (the subsidiary of firm A), which owns $51 \%$ share of company C. However, company $\mathrm{C}$ is actually controlled by company A, which only owns $26.01 \%$ share of company $\mathrm{C}$.
} 
a one standard deviation increase in ownership implies that for an independent director, the probability of being unpaid decreases by $6.46 \%$ but the level of compensation increases by $0.59 \%$; for a non-independent director, the probability of being unpaid increases by $4.36 \%$ and the level of compensation increases by $6.78 \%$. Prior literature suggests that higher ownership of the controlling shareholders leads to greater incentive to monitor the CEO, thereby reducing the rent-extraction and compensation of the CEO (Dyl, 1988, Core, Holthausen, and Larcker, 1999, Cyert, Kang, and Kumar, 2002, Conyon and He, 2011). From this perspective, monitoring due to concentrated ownership may substitute for monitoring from the board of directors. Consistent with this theory, Barontini and Bozzi (2011) find that, in Italy, directors receive less compensation when the ownership is more concentrated. However, our findings that the level of director compensation increases with the ultimate controlling shareholder's ownership suggests that the ultimate controlling shareholders may attract more experts and high-rank bureaucrats to the board with higher compensation.

In Chinese listed firms, the ultimate controlling shareholders often have control rights that exceed their cash-flow rights (measured by ownership). The average divergence between control rights and cash-flow rights is $5.64 \%$. We find no effect of excess control rights on the compensation of independent directors. ${ }^{6}$ However, we find a statistically significant and economically important influence of excess control rights on the compensation of non-independent directors. Specifically, a one standard deviation increase in the excess control rights of the largest shareholders increases the probability of being unpaid by $3.29 \%$ and the level of non-independent director compensation by $7.72 \%$. Our findings are opposite to the literature that suggests that director compensation decreases with the divergence between control rights and ownership (Yeh and Woidtke, 2005, Barontini and Bozzi, 2011).

Related directors are non-independent directors holding positions in both the listed firms and controlling firms. ${ }^{7}$ Because related directors are uncommon in western countries, the literature on the relationship between related directors and compensation is scant. However, related directors are very common in China, where $41 \%$ of nonindependent directors in our sample are related directors. We find that related directors are more likely to be unpaid and receive lower compensation. Specifically, for a nonindependent director that holds a position in a controlling firm, the probability of being unpaid increases by $89.95 \%$ and the level of compensation decreases by $30.65 \%$. Our

\footnotetext{
${ }^{6}$ We measure the excess control rights by the percentage difference between control rights (measured by voting rights) and cash-flow rights (measured by ownership) of the ultimate controlling shareholder.

${ }^{7}$ According to the regulation from China Securities Regulatory Commission (CSRC), an individual holds a position in a controlling firm can not serve as an independent director in the listed firm. Therefore, the related directors in our sample are all non-independent directors.
} 
results are consistent with Lo, Wong, and Firth (2010) who suspect but do not test that a related director is more likely to be unpaid as controlling shareholders may pay part or all of director compensation. To our knowledge, our study is the first work that empirically examines both the level of compensation and the propensity being unpaid for related directors.

The remainder of the paper is organized as follows. Section 2 provides the relevant institutional background in China. Section 3 discusses the related literature and develops the hypotheses. Section 4 describes our sample selection and variable construction. Section 5 presents the empirical method for testing and reports the main empirical results. Section 6 presents the robustness tests. The final section concludes the paper.

\subsection{Institutional background}

\subsubsection{Ownership structure in China}

Ownership structure in China is different from that in the US in several ways. First, state ownership is very common in China. For example, during 2005-2015, almost half (49\%) of the Chinese listed firms are controlled by the government or quasi-state institutions (such as other state-owned companies). Second, ownership is highly concentrated in China. For example, during 2005-2015, the ultimate controlling shareholder owns, on average, over one-third of listed firms, while the five largest shareholders own over half of the firm. Third, institutional ownership is less common in China than in the US. For example, in 2010, institutional investors in China own $16.6 \%$ of tradable shares, while the domestic financial institutions in the US own almost $50 \%$ of US stocks. ${ }^{8}$

State ownership affects firm objectives. First, state-owned companies likely have political objectives in addition to financial objectives. These political objectives include, but are not limited to, maintenance of employment, direct control of important industries such as banking, energy, and telecommunication, and politically motivated job placement (Liu and Lu, 2007). Second, state ownership may lead to soft budget constraints, which arises when the government supports a firm in financial difficulty. Kornai (1980) suggests that the soft budget constraint undermines a firm's incentive to perform productively and efficiently. Taking the state-controlled banking system as the given institutional environment, Che and Qian (1998) develop a model that explains how state-owned companies suffer from the soft budget constraint problem. Because the government controls both

\footnotetext{
${ }^{8}$ Jiang and Kim (2015) believe that the institutional ownership in China is overestimated since nontradable shares are not included in the calculation. In contrast, Edelen, Ince, and Kadlec (2016) believe that the institutional ownership in the US is underestimated since foreign institutional ownership is excluded in the calculation.
} 
the state-owned companies and banks, it can require a bank to refinance the companies for political reasons.

In addition to high levels of state ownership, China has high levels of concentrated ownership. The influence of concentrated ownership (shareholders that hold a high percentage of the firm's stock) on corporate governance is mixed. On one hand, concentrated ownership may improve governance by intensifying monitoring of management. This monitoring story is primarily based on the developed countries literature. In the developed countries such as the US and UK, ownership is normally very dispersed, therefore, the shareholders have limited incentives and powers to monitor the management. ${ }^{9}$ However, this agency problem can be moderated by a large shareholder who has both financial incentives and means to monitor the management (Shleifer and Vishny, 1986, 1997). On the other hand, concentrated ownership may worsen corporate governance. Porta, Lopez-de Silanes, and Shleifer (1999) suggest that in many countries controlling shareholders may expropriate wealth from minority shareholders. ${ }^{10}$ Expropriations include activities ranging from outright theft and fraud to intercorporate loans, loan guarantees for majority shareholders, and selling assets or products below market prices to majority shareholders. In China, controlling shareholders expropriate wealth from minority shareholders mainly through the granting loans and related-party transactions (Liu and Lu, 2007, Jiang, Lee, and Yue, 2010). Some of these practices may add value in ways that counteract the corresponding market frictions. For example, an intercorporate loan may help reduce external financing constraints and transaction costs of the borrowing firms. However, minority investors almost always lose when the controlling shareholder expropriates.

\subsubsection{Board structure in China}

Similar to Germany, China operates under two-tier board system, which includes a director board and a supervisory board. The 1993 Chinese Company Law requires all listed companies to adopt a two-tier board structure, which consists of a director board and a supervisory board. Under the 1993 Chinese Company Law, the director board is a decision-making unit, while the supervisory board is an agency that monitors directors and executives of the company. The supervisory board has the same rank but far less authority than the director board. Unlike the German Supervisory Board, the Chinese Supervisory Board does not appoint or dismiss directors and executives. Rather, the

\footnotetext{
${ }^{9}$ Limited incentives may be due to free rider problems or to relatively high monitoring costs relative to the limited monitoring benefits.

${ }^{10}$ According to Porta, Lopez-de Silanes, and Shleifer (1999), a controlling shareholder does not need to be a majority owner. Actually, they use a $20 \%$ share ownership cutoff to identify the existence of controlling shareholders. Therefore, based on the $20 \%$ threshold, most listed firms in China are likely to have controlling shareholders.
} 
supervisory board monitors the director board and, if anything goes wrong, requires directors and executives to correct their misbehavior. If the misbehavior is not been fixed, the supervisory board may report the misbehavior directly to the regulatory authorities. At the same time, the supervisory board in China bears no legal consequences when the firm goes wrong, limiting its incentives to monitor the directors and executives. ${ }^{11}$ Clarke (2006) suggests that the supervisory board plays no real role in corporate governance. Because of its limited capabilities and incentives, the supervisory board in China is considered ineffective in monitoring, and therefore, Tian (2001) and Tam (1999) suggest that the structure of corporate governance in China is similar to the Anglo-Saxon unitary board rather than the two-tier board.

\subsubsection{The independent director in China}

Ownership structure influences independent director responsibilities. For example, when ownership is dispersed, an important agency problem is the conflict between inside managers and outside shareholders. Correspondingly, an important objective of independent directors is to hold managers accountable for performance. However, when ownership is highly concentrated, an important governance issue is the minimization of wealth expropriation of controlling shareholders from the firm's minority shareholders. Therefore, an important responsibility of independent directors in China is to monitor large controlling shareholders on behalf of minority shareholders. ${ }^{12}$ The China Securities Regulatory Commission (CSRC) provides a legal definition for independent directors. According to the regulation of CSRC, an independent director must not be: (1) an individual who holds a position in the listed company or its subordinate affiliates as well as the direct relatives of, and those with important social connections to, the former; (2) an individual, or the direct relative of an individual, who directly or indirectly holds at least $1 \%$ of the company's share or is among the top ten shareholders of the company; (3) an individual, or the direct relative of an individual, who is employed by an entity that directly or indirectly holds at least $5 \%$ of the company's share or is among the top five non-natural person shareholders of the company; (4) an individual about whom any of the above conditions have been met within the last year; (5) an individual who supplies accounting, legal, consulting, or other similar services to the company or its subordinate affiliates. ${ }^{13}$

\footnotetext{
${ }^{11}$ Through the case study, Dahya, Karbhari, and Xiao (2002) find that supervisors escaped any legal prosecution or penalty in financial scandals.

${ }^{12}$ In 2001, when the independent director system was introduced to Chinese listed firms by CSRC, they explicitly state that the primary and legally explicit responsibility of independent directors is to monitor large controlling shareholders on behalf of minority shareholders. See Guidelines for Introducing Independent Directors to the Board of Directors of Listed Companies 2001.

${ }^{13}$ See Clarke (2006) for the detail discussion on the legal definition of independent directors in China.
} 
To promote the influence of independent directors, the China Securities Regulatory Commission (CSRC) requires that, in listed firms, at least one-third of their board members are independent directors. Also, the CSRC discourages the independent directors from holding the listed firm's shares to protect the independence of the independent directors from controlling shareholders. ${ }^{14}$ Not surprisingly, in China, the equity incentives contracts for independent directors are not allowed and the shareholding of independent directors are extremely low. ${ }^{15}$

However, this regulation may not be fully effective since the controlling shareholders can minimize monitoring by keeping the proportion of independent directors to the minimum one-third required. In our sample, the median proportion of independent directors is one-third, which is exactly the minimum required ratio. ${ }^{16}$ More importantly, controlling shareholders may nominate independent directors and influence director election. According to 2001 guidance opinion, the whole appointment process of independent directors could be divided into three stages: 1) the nomination stage 2) the CSRC check stage 3 ) the selection stage. ${ }^{17}$ At the first stage, director board, supervisory board or the shareholder who holds not less than $1 \%$ of the shares in the listed company could nominate the candidate for independent director. After the nomination, the China Securities Regulatory Commission (CSRC) checks the qualifications and backgrounds of nominees to decide whether the nominees are eligible to serve as the independent directors. ${ }^{18}$ In the end, the shareholder's general meeting elects the independent directors from the eligible candidates for independent director.

\subsection{Literature and hypothesis development}

\subsubsection{Director compensation and director characteristics}

A portion of the literature advances that women executives are underpaid. For example, Fagenson and Jackson (1993) find that in 1992 women executives on average earned 66.2 percent of male executives' compensation in the United States. Likewise, Zelechowski and Bilimoria (2004) find that women non-CEO inside directors earn considerably less than men inside directors. Moreover, Chen, Ezzamel, and Cai (2011) find that in China female

\footnotetext{
${ }^{14}$ For example, the independent directors are not allowed to directly or indirectly hold more than $1 \%$ of the listed firm's shares, nor are they allowed to be one of the top 10 shareholders of the listed firm.

${ }^{15}$ The average shareholding of independent directors in our sample period is lower than $0.01 \%$.

${ }^{16}$ The proportion at the 75 th percentile is only 0.4 , which is barely above the minimum ratio.

${ }^{17}$ See Guidelines for Introducing Independent Directors to the Board of Directors of Listed Companies 2001.

${ }^{18}$ The nominees who fail to meet the CSRC requirement may serve as candidates for company director but not as candidates for independent director.
} 
executives receive approximately $6.7 \%$ less pay compared to male executives. In contrast, Bertrand and Hallock (2001) find that this gap narrows to less than 5\% after controlling for firm size, occupation and job experience. Although there is no literature that explores the propensity for women to be unpaid while sitting on boards, the literature on payment level suggests that the propensity of women to be unpaid should be higher than for men. Thus, our hypothesis is:

H1a: For woman directors, the propensity to be unpaid is higher and the level of compensation is lower, ceteris paribus.

Fama and Jensen (1983) contend that the busyness of an outside director signals the quality of outside director. That is, higher quality outside directors are more frequently offered additional outside directorships. Recent literature provides further empirical evidence that higher quality directors are more sought after and that the quality of director is positively related to busyness (Gilson, 1990, Kaplan and Reishus, 1990, Brickley, Linck, and Coles, 1999, Coles and Hoi, 2003, Brown and Maloney, 1999, Fich and Shivdasani, 2007). Moreover, Field, Lowry, and Mkrtchyan (2013) suggest that the connections and experience of busy directors make them better advisors. If the busyness of director serves a good proxy for the quality of director, then the busy director demands and receives higher compensation. Therefore, our hypothesis is:

H1b: For busy directors, the propensity to be unpaid is lower and the level of compensation is higher, ceteris paribus.

Dou, Sahgal, and Zhang (2015) suggest that the tenure of directors may serve as a proxy for their ability. They provide three reasons. First, more experienced directors work with multiple CEOs, which aids them in assessing the ability of the current CEO. Second, directors with long tenure have a larger financial stake in the company than their short tenure counterparts, aligning their interests with that of the shareholders. Third, longer tenure periods certify the position of the director, helping her balance the CEO's influence in the boardroom. Consistent with the positive effect of tenure, Dou, Sahgal, and Zhang (2015) find that the directors with longer tenure attend more meetings and serve on more committees. Moreover, CEOs in firms with a larger number of experienced directors tend to have lower compensation and are more likely to leave when the firm performs poorly. These firms are also less likely to restate earnings and make acquisitions (and those that are made are more likely to be profitable). Overall, Dou, Sahgal, and Zhang (2015) evidence that the boards with a higher proportion of experienced directors are better at both monitoring and advising. If the tenure of director serves a good proxy for the quality of director, then the director with longer tenure should receive higher compensation. However, Chen, Ezzamel, and Cai (2011) find that in China the 
compensation of top 3 executives is negatively related to their tenure. Our hypothesis is therefore:

H1c: For directors with long tenure, the propensity to be unpaid is lower and the level of compensation is higher, ceteris paribus.

\subsubsection{Compensation and ownership structure}

The literature finds that ownership structure influences CEO compensation. Firth, Fung, and Rui (2007) find that the CEOs in Chinese listed firms receive less compensation in state-owned firms than their counterparts in non-state-owned firms. This difference in compensation arises because the CEOs of state-owned firms are often state bureaucrats and their compensation aligns with the senior officer salary levels. Liang, Renneboog, and Sun (2015) find that state ownership not only reduces the level of executive compensation but also increases pay-for-performance sensitivity in China. Likewise, Barontini and Bozzi (2011) find that director compensation is lower when the firms are state-owned in Italy. Chen, Luo, and Soderstrom (2016) document that the Chinese government imposes a cap on executive compensation in state-owned firms companies due to social concerns. The evidence that salary levels of CEOs are lower in state-owned firms suggests that same relationship holds for members of the board of directors. This evidence leads us to conjecture that:

H2a: For directors who serve in the state-owned companies, the propensity to be unpaid is higher and the level of compensation is lower, ceteris paribus.

When the controlling shareholders have more ownership, they are motivated to monitor the CEOs and top management (Dyl, 1988, Core, Holthausen, and Larcker, 1999, Cyert, Kang, and Kumar, 2002, Claessens, Djankov, Fan, and Lang, 2002). Monitoring due to concentrated ownership may substitute for monitoring from the board of directors. In the substitution case, director compensation decreases. Consistent with the substitution case, directors in European countries receive less compensation when the ownership is more concentrated (Barontini and Bozzi, 2011, Andreas, Rapp, and Wolff, 2012). In addition to monitoring, the board of directors may provide advisory or political connections. Experts and influential politicians add value to corporations. If controlling shareholders are motivated to increase value, they may appoint more experts and highrank bureaucrats to the board, increasing the director compensation. Therefore, the relationship between the ownership of ultimate controlling shareholders and compensation is an empirical question. Basing on the idea that ownership concentration and board monitoring are substitutions, we posit the following hypothesis:

H2b: For directors who serve in firms with highly concentrated owners, the propensity to be unpaid is higher and the level of compensation is lower, ceteris paribus. 
Claessens, Djankov, Fan, and Lang (2002) find that concentrated owners East Asian countries may have control rights that exceed their cash-flow rights in the firms. Firm value falls when the control rights of the ultimate controlling shareholder exceed their cash-flow rights. Claessens, Djankov, Fan, and Lang (2002) suggest this decrease of firm value is due to the risk of expropriation of minority shareholders by controlling shareholders. Yeh and Woidtke (2005) suggest that controlling shareholders influence the board selection process and, when the divergence between control rights and cash flow rights is higher, controlling shareholders may select both board members that are more likely to make decisions favoring controlling shareholders and those that are less likely to monitor. Moreover, Barontini and Bozzi (2011) find that, in Italy, the directors receive less compensation when the divergence between control rights and cash flow rights is higher. This evidence leads us to conjecture that:

H2c: For directors in firms where the divergence between control and cash flow rights is large, the propensity to be unpaid is higher and the level of compensation is lower, ceteris paribus.

Chen, Luo, and Soderstrom (2016) find that a large number of CEOs in state-owned firms are paid directly by the Chinese government (or parent firms controlled by the Chinese government) rather than by companies for which they work. Likewise, Lo, Wong, and Firth (2010) suspect that directors who hold positions in both the listed firm and the parent firm are paid part or all by the controlling shareholders. Our hypothesis is therefore:

H2d: For directors who hold positions in a related firm, the propensity to be unpaid is higher and the level of compensation is lower, ceteris paribus.

\subsection{Data and variable construction}

\subsubsection{Sample construction}

The sample for this study consists of all firms listed on the Shanghai Stock Exchange (SSE) and Shenzhen Stock Exchange (SZSE) for the period 2005-2015. We choose the sample period from 2005 to 2015 since the director' compensation information is not reported at the individual level until 2005. The improved reporting is a result of a regulation by the China Securities Regulatory Commission (see CSRC (2005)), which requires listed firms to report compensation information for each individual executive beginning in 2005. The board composition, director profile, equity ownership, director meeting attendance and accounting data are collected from the Chinese Listed Firms Research Series database and the stock price data are collected from the China Stock Market Series 
database, which both are the subsets of China Stock Market and Accounting Research (CSMAR) database. ${ }^{19}$ The director profile data contains information on director compensation and director characteristics, such as the director's tenure, gender, age and director shareholding.

To construct a director-level dataset, we merge director profile data with director meeting attendance data by year, stock code and director name. We then merge this director-level dataset with equity ownership, board composition, and accounting data by year and stock code. We drop observations where values of total compensation are missing. Our final sample consists of 226,322 director-firm years, and 2,893 firms, whose number varies from 1,375 in 2005 to 2,843 in 2015.

\subsubsection{Dependent variables}

To test what explains the propensity to be unpaid, we use the variable $\operatorname{Unpaid}(0 / 1)_{t}$ as the dependent variable. A value of 1 is assigned if a director does not receive compensation in a given period and 0 otherwise. Table 1.1 shows that $6 \%$ independent directors and $36 \%$ non-independent directors are unpaid. We categorize our sample into independent directors and non-independent directors using the classification from the CSMAR database, which is a legal definition of director independence. ${ }^{20}$ To test what determines the compensation level, we use the variable $\operatorname{Ln}(\text { Compensation })_{t}$ as the dependent variable. Ln(Compensation) $)_{t}$ is the natural logarithm of compensation of a director in a given period. Table 1.1 shows that the average annual compensation is about 57,654 CNY (equivalent to 8,478 USD with the exchange rate of $6.8 \mathrm{CNY} / \mathrm{USD}$ ) for an independent director and 288,193 CNY (equivalent to 42,381 USD with the exchange rate of 6.8 CNY/USD) for a non-independent director in China, which are far less than that of their counterparts at developed countries.

\subsubsection{Variables of interest}

To test how director characteristics affect compensation, we construct several director characteristics variables. Woman $(0 / 1)$ is a dummy variable that equals to 1 if the director is female and 0 otherwise. Busy director $(0 / 1)_{t}$ is a dummy variable that equals to 1 if the independent director holds more than two directorships and 0 otherwise. The

\footnotetext{
${ }^{19}$ The CSMAR database is widely regarded as the most comprehensive and authoritative database to study corporate finance and corporate governance in Chinese listed firms. According to a report issued by Shenzhen GTA, the CSMAR database has been used in papers published in a dozen leading international journals including Journal of Finance, Journal of Financial Economics, Journal of Financial and Quantitative Analysis and Review of Financial Studies.

${ }^{20}$ See Section 1.2.3 for the legal definition of director independence.
} 
multiple directorships of independent directors could serve as a proxy for director capacity. $^{21}$ Tenure $_{t}$ measures the number of years a director has served on the board. Table 1.1 shows that the number of directorships and the proportion of female directors in the independent director sample are similar to those in the Adams and Ferreira (2008). The non-independent director sample, however, includes fewer female and additional directorships. On average, in our sample, the independent director is 53 years old and has 6.11 years working experience as a director and the non-independent director is 49 years old and has 5.9 years working experience as a director, which are both younger and less experienced than those in the existing literature. ${ }^{22}$

To test how ownership structure affects the director compensation, we construct several measures of ownership structure. State-owned $(0 / 1)_{t}$ is a dummy variable equals to 1 if the firm is state-owned and 0 otherwise. Table 1.1 shows that $48 \%$ of the firms in our

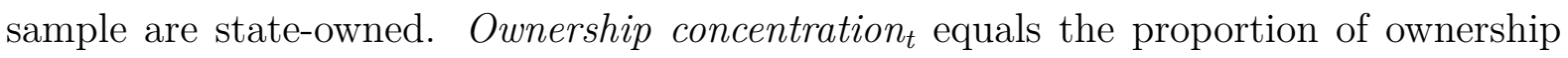
(cash-flow rights) held by the ultimate controlling shareholders. Excess control right measures the difference between control rights and ownership of the ultimate controlling shareholders. Table 1.1 shows that the ultimate controlling shareholders of listed firms in China have high ownership. Specifically, the ultimate controlling shareholders on average own $33 \%$ share from the listed firms. Such concentrated ownership may emphasize that the main conflicts in Chinese listed firms are not between shareholders and the manager but between the ultimate controlling shareholders and the minority shareholders. For the ultimate controlling shareholders, the divergence between control rights and cash flow rights is about $6 \%$ on average, which is similar to that in Chou, Chung, and Yin (2013). We use related director to measure the connection to large shareholders, where Related director $(0 / 1)_{t}$ is a dummy variable that equals to 1 if the non-independent director holds a position in the controlling firm and 0 otherwise. Related directors are very common in China, where $41 \%$ of non-independent directors in our sample are related directors.

\subsubsection{Control variables}

For all regressions, we control for director, board and firm features. The director level control variables include $C E O / C O B(0 / 1)_{t}, A g e_{t}, A g e_{t}^{2}$, Meeting frequency $(I d)_{t}$, Meeting frequency(Firm) $t_{t}$, and Ln(Share ownership +1$)_{t}$, where $C E O / C O B(0 / 1)_{t}$, Meeting frequency $(\text { Firm })_{t}$, and $\operatorname{Ln}(\text { Share ownership }+1)_{t}$ are applied only to non-independent di-

\footnotetext{
${ }^{21}$ In China, non-independent directors may simply hold multiple directorships since they serve as the proxies for controlling shareholders in several firms. Thus, the multiple directorships of non-independent directors may not signal director quality.

${ }^{22}$ For example, the age, and tenure of outside directors in Adams and Ferreira (2008) is 60 and 9.63.
} 
rectors and Meeting frequency $(I d)_{t}$ is applied only to independent directors. The board level control variables include $L n(C E O \text { compensation }+1)_{t}$, Dualityt, Board size $_{t}$ and Board composition(Ind\%)t. The firm-level control variables include Cash holdings ${ }_{t}$, Ln(Total Assets $)_{t}$, Book leverage,$R_{t} O A_{t-1}$ and Stock volatility $t-1 .{ }^{23}$ We also control for industry and year in all regressions. Table 1.9 provides a summary of all variable definitions.

Table 1.1 shows that the average board in China has about nine members. However, only $37 \%$ of them are independent directors. This ratio is far below than that observed in developed countries literature and only slightly above the required ratio from the China Securities Regulatory Commission (CSRC). ${ }^{24}$ On average, the board has 8.38 meetings a year for independent directors and 9.49 meetings for directors. The duality is about 0.21. For the firm characteristics, the average size of firm is about 42.09 billion CNY (6.19 billion USD), however, this figure is unrepresentative for Chinese listed firms since the median and upper 25th percent sample are far smaller than the mean, where these two figures equals to 2.74 billion CNY (0.4 billion USD) and 6.7 billion CNY (0.99 billion USD) respectively. The average book leverage for Chinese listed firms is $53 \%$. The average ROA of Chinese listed firms is $3 \%$. The average rate of cash holding for Chinese listed firms is $16 \%$ and the annual volatility of stock share is 0.15 . Table 1.2 provides the correlation matrix for the key variables in the whole sample. Panel A provides pairwise correlation coefficients for the non-independent directors and Panel B provides pairwise correlation coefficients for independent directors. As expected, there are negative correlation coefficients between being unpaid and compensation level.

\subsection{Testing approach and results}

\subsubsection{Testing approach}

The general structure of our testing model is:

$$
Y_{i j t}=f\left(\alpha+\mathbf{C H A R} \delta+\mathbf{O W N} \lambda+\mathbf{X} \beta+\epsilon_{i j t}\right),
$$

where $i$ represents the director, $j$ the firm, and $t$ the year. The dependent variable $Y_{i j t}$ is either Unpaid(0/1) $t$ or $\operatorname{Ln}(\text { Compensation })_{t}$. The functional form $f($.$) is logistic$ when the dependent variable is $\operatorname{Unpaid}(0 / 1)_{t}$ and linear when the dependent variable is $L n$ (Compensation) $t$. CHAR represents a matrix of director characteristic variables

\footnotetext{
${ }^{23}$ Similar to Chou, Chung, and Yin (2013) the ROA is the annual return on book assets and the volatility is the variance of monthly stock returns. For both ROA and volatility, we lagged them for one year.

${ }^{24}$ The China Securities Regulatory Commission (CSRC) required the listed firms in China have at least $1 / 3$ of their board members to be independent directors.
} 
including $\operatorname{Woman}(0 / 1)$, Busy director $(0 / 1)_{t}$ and Tenure $_{t}$. OWN represents a matrix of ownership structure variables including State-owned(0/1) , Ownership concentration $_{t}$, Excess control right $t_{t}$ and Related director $(0 / 1)_{t}$. $\mathbf{X}$ represents a matrix controls variables including director age, director share ownership, board characteristics variables, firm characteristics variables, year and industry. Lastly, $\alpha$ represents the constant term in the regression and $\epsilon_{i j t}$ is the error term.

\subsubsection{Director compensation and characteristics}

Table 1.3 tests the propensity to receive zero compensation and the level of compensation for both independent and non-independent directors. Columns (1) and (2) test using the sample of independent directors. Columns (3) and (4) test using the sample of nonindependent directors. Columns (1) and (3) test using a logit regression model where the dependent variable is the propensity to received zero compensation. ${ }^{25}$ Columns (2) and (4) test using an OLS regression model where the dependent variable is the natural logarithm of compensation.

Table 1.4 reports the economic importance of the variables of interest on both the propensity to receive zero compensation and the level of compensation. Columns (1) and (2) report the predicted sign of regression coefficients. Columns (3) and (4) report the percentage change of both Unpaid(0/1) $t$ and the level of compensation from their mean values for independent directors when a one standard deviation increase (from the mean value) in continuous variables and one unit increase (from 0 to 1 ) in dummy variables. Columns (5) and (6) report the percentage change of both Unpaid(0/1) $t$ and the level of compensation from their mean values for non-independent directors when a one standard deviation increase (from the mean value) in continuous variables and one unit increase (from 0 to 1 ) in dummy variables.

\section{Compensation and gender}

Table 1.3 reports that the coefficient associated with $\operatorname{Woman}(0 / 1)$ is statistically no different than zero in explaining both the propensity to receive zero compensation and the level of compensation for an independent director. In contrast, Column (3) reports that the coefficient associated with $\operatorname{Woman}(0 / 1)$ is negative and statistically significant at the $10 \%$ level in explaining the propensity to receive zero compensation for a non-independent director. Column (4) reports that the coefficient associated with Woman(0/1) is statisti-

\footnotetext{
${ }^{25}$ We do not present the results of probit regression model since they are similar to those of logit regression model.
} 
cally no different than zero in explaining the level of compensation of a non-independent director.

Table 1.4 shows the economic importance of $\operatorname{Woman}(0 / 1)$ on director compensation. ${ }^{26}$ For non-independent directors, the propensity to be unpaid decreases $4.39 \%$ from its mean $(35.57 \%)$ for a female director relative to a male director. Overall, we find no evidence that women directors are underpaid relative to their male colleagues in China. Therefore, our results reject Hypothesis H1a that female directors have both a higher propensity to receive zero compensation and receive a lower level of compensation.

\section{Compensation and busy independent director}

Column (1) in Table 1.3 reports that the coefficient associated with Busy director $(0 / 1)_{t}$ is negative and statistically significant at less than the $1 \%$ level in explaining the propensity to receive zero compensation. Column (2) reports that the coefficient associated with Busy director $(0 / 1)_{t}$ is positive and statistically significant at less than the $1 \%$ level in explaining the level of compensation. Our evidence suggests that the busy directors are less likely to be unpaid and receive a higher level of compensation, and thereby supports Hypothesis H1b. Table 1.4 reports the estimated economic impact of Busy director $(0 / 1)_{t}$ on director compensation. For busy directors, the propensity to receive zero compensation decreases $11.18 \%$ from its mean $(6.11 \%)$ and the level of compensation increases $2.78 \%$ from its mean (57.65 Thousand $C N Y$ ). We do not apply the busy director concept to non-independent directors with multiple directorships since, in China, the multiple directorships of non-independent directors could be mechanical. A non-independent director may simply hold multiple directorships in several firms since he or she is the proxy for the controlling shareholder. Thus, in China, the multiple directorships of non-independent directors are not a good proxy for director quality and do not fit the busy director literature.

\section{Compensation and director tenure}

Columns (1) and (3) in Table 1.3 report that the coefficients associated with Tenure $_{t}$ are negative and statistically significant at less than the $1 \%$ level in explaining the propensity to receive zero compensation for both independent directors and non-independent directors. In contrast, Columns (2) and (4) in Table 1.3 report that the coefficients associated with Tenure $_{t}$ are positive and statistically significant at less than the $1 \%$ level in explaining the level of compensation for both independent directors and non-independent

\footnotetext{
${ }^{26}$ We only report here the marginal effect of $\operatorname{Woman}(0 / 1)$ on the propensity to be unpaid for nonindependent directors since other coefficients associated with $\operatorname{Woman}(0 / 1)$ are statistically no different than zero.
} 
directors. Therefore, our results support Hypothesis H1d that directors with longer tenure have both a lower propensity to receive zero compensation and receive a higher level of compensation.

Table 1.4 reports the estimated economic impact of Tenure $_{t}$ on director compensation. For independent directors, a one standard deviation increase in Tenure $_{t}$ decreases Unpaid(0/1) t by $13.53 \%$ from its mean $(6.11 \%)$ and increases the level of compensation by $1.62 \%$ from its mean (57.65 Thousand $C N Y$ ). For non-independent directors, a one standard deviation increase in Tenure $_{t}$ decreases Unpaid $(0 / 1)_{t}$ by $11.68 \%$ from its mean (35.57\%) and increases the level of compensation by $13.94 \%$ from its mean (288.19 Thousands $C N Y$ ).

\subsubsection{Director compensation and ownership structure}

\section{Compensation and state ownership}

Column (1) in Table 1.3 reports that the coefficient associated with State-owned $(0 / 1)_{t}$ is positive and statistically significant at less than the $10 \%$ level in explaining the propensity to receive zero compensation for independent directors. ${ }^{27}$ Column (3) reports that the coefficient associated with State-owned $(0 / 1)_{t}$ is positive and statistically significant at less than the $1 \%$ level in explaining the propensity to receive zero compensation for nonindependent directors. Columns (2) and (4) in Table 1.3 report that the coefficients associated with State-owned $(0 / 1)_{t}$ are negative and statistically significant at less than the $1 \%$ level in explaining the level of compensation for both independent directors and non-independent directors. Overall, our evidence suggests that the directors working in a state-owned firm are more likely to be unpaid and receive lower level of compensation. Therefore, our results support our Hypothesis H2a that directors working in the stateowned companies have both a higher propensity to receive zero compensation and receive a lower level of compensation.

Table 1.4 reports the estimated economic impact of State-owned $(0 / 1)_{t}$ on director compensation. For independent directors, the level of compensation decreases $7.92 \%$ from its mean (57.65 Thousand CNY) if a firm shifts from non-state owned to state-owned. For non-independent directors, the propensity to receive zero compensation increases $31.20 \%$ from its mean $(35.57 \%$ ) and the level of compensation decreases $11.57 \%$ from its mean (288.19 Thousands CNY) if a firm shifts from non-state owned to state owned.

\footnotetext{
${ }^{27}$ The analysis from mixed effect regressions suggest that the positive relation between state-ownership and propensity of independent directors to be unpaid is likely driven by omitted variables in director level.
} 


\section{Compensation and ownership concentration}

Ownership concentration affects director compensation differently over the types of directors. Columns (1) and (3) in Table 1.3 report that, in explaining the propensity to receive zero compensation, the coefficient associated with Ownership concentration t $_{\text {is negative }}$ and statistically significant at less than the $1 \%$ level for independent directors but positive and statistically significant at less than the $1 \%$ level for non-independent directors. Columns (2) and (4) in Table 1.3 report that in explaining the level of compensation, the

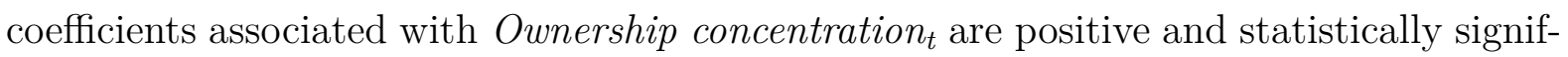
icant at less than the $5 \%$ level for independent directors and non-independent directors. Our results suggest that, when the ultimate controlling shareholders have more cash-flow rights, the independent directors are less likely to be unpaid while the non-independent directors are more likely to be unpaid. However, the level of director compensation is increasing with the cash-flow rights of ultimate controlling shareholders. Our results reject the Hypothesis $\mathrm{H} 2 \mathrm{~b}$ that directors are more likely to be unpaid and receive a lower level of compensation when the ownership is more concentrated in the case of independent director. In addition, our results partially support Hypothesis $\mathrm{H} 2 \mathrm{~b}$ in the case of the non-independent director. Overall, our evidence does not support the previous studies finding that the directors receive less compensation when the ownership is more concentrated (Barontini and Bozzi, 2011).

Table 1.4 reports the estimated economic impact of Ownership concentration $n_{t}$ on director compensation. For independent directors, the propensity to receive zero compensation decreases $6.46 \%$ from its mean $(6.11 \%)$ and the level of compensation increase $0.59 \%$ from its mean ( 57.65 Thousand $C N Y$ ) if the ownership of the ultimate controlling shareholder's increases by one standard deviation. For non-independent directors, the propensity to be unpaid increases $4.36 \%$ from its mean $(35.57 \%$ ) and the level of compensation increases $6.78 \%$ from its mean (288.19 Thousands CNY) if the ownership of the ultimate controlling shareholder's increases by one standard deviation. Additionally, our evidence suggests board function and ownership concentration are compliments and not substitutions.

\section{Compensation and excess control rights}

Columns (1) and (2) in Table 1.3 report that the coefficients associated with Excess

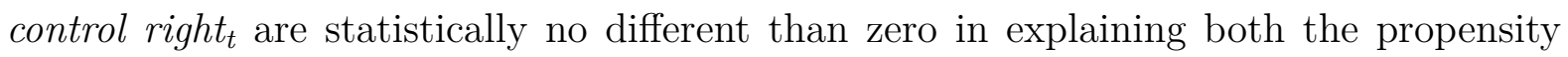
to receive zero compensation and the level of compensation for an independent director. Also, Columns (3) and (4) in Table 1.3 report that, for a non-independent director, the coefficients associated with Excess control right $t_{t}$ are positive and statistically significant 
at less than the $1 \%$ in explaining both the propensity to receive zero compensation and the level of compensation. Our results reject our Hypothesis H2c that director is more likely to be unpaid and receives a lower level of compensation when the excess right is bigger in the case of independent directors and partially support our Hypothesis H2c in the case of non-independent directors.

Table 1.4 reports the estimated economic impact of Excess control right $t_{t}$ on director compensation. For a non-independent director, a one standard deviation increase in

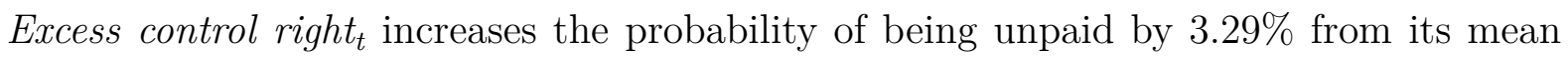
$(35.57 \%)$ and the level of compensation by $7.72 \%$ from its mean (288.19 Thousands $C N Y)$.

\section{Compensation and related non-independent director}

Column (3) in Table 1.3 reports that the coefficient associated with Related director $(0 / 1)_{t}$ is positive and statistically significant at the $1 \%$ level in explaining the propensity for a non-independent director to receive zero compensation. Besides, Column (4) in Table 1.3 reports that the coefficient associated with Related director $(0 / 1)_{t}$ is negative and statistically significant at the $1 \%$ level in explaining the level of compensation. The results indicate that the directors related to the controlling shareholders are more likely unpaid and receives a lower level of compensation. Therefore, our results support Hypothesis H1c that related directors are more likely to be unpaid and receive a lower level of compensation.

Table 1.4 reports the estimated economic impact of Related director $(0 / 1)_{t}$ on director compensation. For related directors, the propensity to receive zero compensation increases $89.95 \%$ from its mean (35.57\%) and the level of compensation decreases $30.65 \%$ from its mean (288.19 Thousand CNY).

\subsection{Robustness tests}

\subsubsection{Zero pay and low pay}

To verify whether the zero compensation is a data issue, we randomly choose 30 firms containing unpaid directors and compare the compensation data from CSMAR with those from annual reports. We find that the compensation data from CSMAR is matched with those from annual reports.

In Table 1.3, Columns (1) and (2) report that the coefficients associated with Ln(Total Assets) ${ }_{t}$ are positive and statistically significant at the $1 \%$ level in explaining both the propensity to receive zero compensation and the level of compensation for an independent 
director. The reputation story may explain the above result. Compared with a small firm, a large firm has more visibility on media. Therefore, for those directors focusing on reputation, they are more willing to take an unpaid directorship in large firms than small firms since the directorship from large firms provides higher reputation values than that from small firms. On the other hand, for those directors concerned more about monetary rewards, large firms are more capable of paying them a higher level of compensation. Our findings suggest that being unpaid could be fundamentally different from receiving a low pay.

\subsubsection{Tenure and new director}

Our results between compensation and tenure suggest a linear relationship. It is possible that our results regarding tenure and compensation could be driven by new directors. Similar to interns that accept an unpaid or underpaid position, a new director may accept an unpaid or underpaid position to gain board experience. To test this assumption, we include New director $(0 / 1)_{t}$ as the alternative measure of director tenure. New director $(0 / 1)_{t}$ is a dummy variable equals to 1 if an individual serves as a board of director for the first time and 0 otherwise. Besides, we include Tenure $e_{t}^{2}$ to test whether the relationship between director compensation and tenure is linear.

Table 1.5 provides the regression results of Tenure Tenure $_{t}^{2}$ and New director $(0 / 1)_{t}$. Columns (1) and (5) report that, for both independent directors and non-independent directors, the coefficients associated with Tenure $_{t}$ are negative and statistically significant at less than the $1 \%$ level in explaining the propensity to be unpaid but positive and statistically significant at less than the $1 \%$ level in explaining the level of compensation. Columns (2) and (6) report that the coefficients associated with Tenure $_{t}^{2}$ are opposite to the coefficients associated with Tenure $_{t}$, suggesting a nonlinear relationship between director compensation and director tenure. Columns (3) and (7) report the regression results of New director $(0 / 1)_{t}$. The coefficients associated with New director $(0 / 1)_{t}$ are positive and statistically significant at less than the $1 \%$ level in explaining the propensity to be unpaid but negative and statistically significant at less than the $1 \%$ level in explaining the level of compensation. The above results indicate that directors who have no previous board experience are more likely to be unpaid and receive less compensation. Columns (4) and (8) report the regression results of Tenure $t_{t}$ and Tenure $_{t}^{2}$ when New director $(0 / 1)_{t}$ is included. For independent directors, when New director $(0 / 1)_{t}$ is included, the coefficients associated with Tenure $_{t}$ and Tenure $_{t}^{2}$ in explaining the propensity to receive zero compensation become statistically no different than zero, while the coefficients associated with Tenure $_{t}$ and Tenure $_{t}^{2}$ in explaining the level of compensation 
change to the opposite signs. However, there is no change in the coefficients of Tenure $_{t}$ and Tenure $_{t}^{2}$ for non-independent directors when New director $(0 / 1)_{t}$ is included.

\subsubsection{Political connections and compensation}

If political connections benefit the firm, a board of member with a political background may receive higher compensation. To test, we include Political background $(0 / 1)_{t}$ as the measure of director's political connections. Political background $(0 / 1)_{t}$ is a dummy variable that equals to 1 if a board of director had or has an administrative ranking in Chinese political system and 0 otherwise. ${ }^{28}$ We source data on director's political background from the China Stock Market and Accounting Research (CSMAR) database. Political connection data in CSMAR is only available after 2008. Therefore, our sample size drops from 60,231 to 47,214 for independent directors and 95,160 to 74,440 for non-independent directors. Table 1.6 provides the regression results of the coefficients associated with $\mathrm{Po-}$ litical background(0/1) . Columns (1) and (2) report results for independent directors. Columns (3) to (4) report results for non-independent directors. Panel A reports results for directors working in state-owned firms. Panel B reports results for directors working in non-state-owned firms. Because the other coefficients associated with the other variables of interest remain qualitatively unchanged, we only report the coefficient associated with Political background $(0 / 1)_{t}$.

Panel A reports that, in state-owned firms, the coefficients associated with Political background $(0 / 1)_{t}$ for independent directors are positive and statistically significant at less than the $10 \%$ level in explaining the propensity to be unpaid and level of compensation. The above results indicate that, in state-owned firms, independent directors with political background are more likely to be unpaid but receive more compensation when paid. The result of unpaid may be driven by the anti-corruption regulation from the Central Commission for Discipline Inspection (CCDI) at 2008. ${ }^{29}$ Panel B reports that in nonstate-owned firms the coefficients associated with Political background(0/1)t for both independent and non-independent directors are positive and statistically significant at less than the $10 \%$ level in explaining the level of compensation. The above results indicate that in non-state-owned firms directors with political background receive higher compensation.

\footnotetext{
${ }^{28}$ Our political measure is broader than that of Liang, Renneboog, and Sun (2015) since it includes not only the officials from government or military but also individuals from other political institutions such as the National People's Congress (NPC), National Committee of the Chinese People's Political Consultative Conference (CPPCC) and eight democratic parties.

${ }^{29}$ In 2008, the Central Commission for Discipline Inspection (CCDI) issues Regulation on Resigned or Retired Senior Cadres Working as Independent Directors or Independent Supervisor in Public Listed Firm or Fund Management Company. Under this regulation, resigned senior officers are allowed to receive compensation from the listed firms, while retired senior officers are not allowed to receive compensation from the listed firms.
} 
This finding is consistent with directors with political backgrounds increasing firm value through their political connections. For example, Correia (2014) find that in the US firms with political connections are less likely to be involved in SEC enforcement and face lower penalties if they are prosecuted by the SEC. Likewise, Houston, Liangliang, Chen, and Ma (2014) find that in the US the political connections of listed firms reduce their cost of debt, and therefore, increase the value of firms.

\subsubsection{Time invariant director heterogeneity}

If there exist unobservable time-invariant director characteristic that is correlated with our variables of interest, then our estimation results may be biased. Therefore, we include director effects to control for director heterogeneity. Since gender and other time-invariant director characteristics are perfectly correlated, we can not use director fixed effects. Rather, we estimate a hierarchical model using Equation (1.2):

$$
Y_{i j t}=f\left(\alpha+\mathbf{C H A R} \delta+\mathbf{O W N} \lambda+\mathbf{X} \beta+\mu_{i}+\epsilon_{i j t}\right),
$$

where $\mu_{i}$ is a director effect. Because $\mu_{i}$ is perfectly correlated with gender, we can not use gender and director fixed effects. Rather, we estimate a hierarchical model where $\mu_{i}$ is a random intercept, which controls for time invariant director heterogeneity.

Table 1.7 provides the regression results. Most of the results from the hierarchical model are similar to the results from baseline model, suggesting that the results from baseline model are robust to the director effect. However, a few of the coefficients change the signs or level of significance. Specifically, when director effect is included, the coefficient associated with State-owned $(0 / 1)_{t}$ in explaining the propensity to be unpaid of an independent director changes from positive and statistically significant at less than the $10 \%$ level to statistically no different than zero.

\subsubsection{Ownership concentration and compensation (state-owned versus non-state-owned)}

The state-owned and non-state-owned firms may have different objectives. Therefore, the influence of Ownership concentration ${ }_{t}$ on director compensation may vary between state-owned and non-state-owned firms. To test, we separate the whole sample into stateowned and non-state-owned sub-samples. Table 1.8 provides the regression results by sub-samples. Columns (1) and (2) report results for independent directors. Columns (3) to (8) report results for non-independent directors. Panel A reports results for directors 
working in state-owned firms. Panel B reports results for directors working in non-stateowned firms.

Our baseline results suggest that, when the controlling shareholders have more cashflow rights, independent directors are less likely to be unpaid and receive a higher level of compensation. However, the regression results by state-owned and non-state-owned sub-samples suggest that this positive relationship between independent director compensation and ownership concentration only holds for non-state-owned firms. In addition, our baseline results show that when the ultimate controlling shareholder has higher cashflow rights, non-independent directors are more likely to be unpaid but receive a higher level of compensation. The sub-sample regressions show that the positive relationship between ownership concentration and the propensity of non-independent directors to be unpaid only holds for state-owned firms. Further, the positive relationship between director compensation and ownership concentration only holds for a non-independent director without either CEO or COB title.

\subsection{Conclusion}

Although director compensation has been researched in the US, the topic is underresearched in general and in particular in China, where the ownership structure and governance issues differ from those in US. ${ }^{30}$ Therefore, our study of director compensation in China fills this gap. Importantly, our study finds both similarities and differences between US and Chinese board of director compensation.

Our study suggests that the relationship between personal characteristics and board compensation are similar in both Chinese and western boards. Both ability (measured by director busyness) and experience (measured by board tenure) positively explain compensation. However, our study helps explain why a substantial percentage of the board members in China are ostensibly unpaid. Although we can't rule out that compensation is received indirectly, we show that the propensity for a director to be unpaid is related to tenure and in particular the unpaid effect is driven by new directors. This is consistent with an inexperienced director accepting a position with the expectation of future pay, which is similar to the idea of an internship. Lastly, we find that, in China, female independent directors are not underpaid, while female non-independent directors are less likely to be unpaid. Although the US evidence suggests woman tend to be underpaid, the economic importance drops with additional controls. Lastly, in robustness testing we show that directors in non-state owned firms with political connections have a lower

\footnotetext{
${ }^{30}$ Brick, Palmon, and Wald (2006) suggest that director compensation only recently received attention in the US.
} 
propensity to be unpaid and a higher level of compensation, a result which is consistent with studies using US data. Overall, the relationship between compensation and ability (busyness), tenure (experience), political connections, and gender and in Chinese boards are similar to western boards. However, the board intern like practice appears unique to the Chinese system.

Our study suggests that director compensation practices that differ from western practices are shaped by different ownership structures. For example, the board of directors serving in the state-owned firms are more likely to be unpaid and receive less compensation. This finding is consistent with SOEs objectives (such as employment) rather than exclusively wealth maximization. In contrast, board members receive higher compensation when controlling shareholders have a higher economic stake (measured by cash-flow rights) on the listed firms. As discussed above, another unique feature in Chinese boards is the practice of ostensibly paying zero compensation to the board members. This empirical regularity is consistent with large shareholders influencing board appointments. In particular, the large shareholders of the listed firms appoint a significant proportion of non-independent directors from the institutions or firms controlled by the large shareholders. These related directors are far more likely to be unpaid from the listed firms for their board service. In our sample, $59.4 \%$ of related directors receive zero compensation from the listed firm for their board service, whereas only $20.4 \%$ of unrelated directors receive zero compensation. Due to data limitations, we can't rule out that related directors are paid by their controlling institution and the zero pay is due to measurement error; however, our finding is also consistent with controlling shareholders not funding good governance through the board. A natural extension of the paper is to test the relationship between ownership, unpaid directors, and tunneling. Overall, the differences in director compensation between Chinese and western boards reflect a mixture of diverse and concentrated ownership structure. 


\section{Bibliography}

Adams, Renée B., and Daniel Ferreira, 2007, A theory of friendly boards, The Journal of Finance 62, 217-250.

, 2008, Do directors perform for pay?, Journal of Accounting and Economics 46, $154-171$.

Adams, Renée B., Benjamin E. Hermalin, and Michael S. Weisbach, 2010, The role of boards of directors in corporate governance: A conceptual framework and survey, Journal of Economic Literature 48, 58-107.

Andreas, Jörn M., Marc Steffen Rapp, and Michael Wolff, 2012, Determinants of director compensation in two-tier systems: evidence from German panel data, Review of managerial science $6,33-79$.

Barontini, Roberto, and Stefano Bozzi, 2011, Board compensation and ownership structure: empirical evidence for Italian listed companies, Journal of Management \& Governance 15, 59-89.

Bertrand, Marianne, and Kevin F. Hallock, 2001, The gender gap in top corporate jobs, Industrial and Labor Relations Review 55, 3-21.

Brick, Ivan E., Oded Palmon, and John K. Wald, 2006, Ceo compensation, director compensation, and firm performance: Evidence of cronyism?, Journal of Corporate Finance 12, 403-423.

Brickley, James A., James S. Linck, and Jeffrey L. Coles, 1999, What happens to CEOs after they retire? New evidence on career concerns, horizon problems, and CEO incentives, Journal of Financial Economics 52, 341-377.

Brown, William O., and Michael T. Maloney, 1999, Exit, voice, and the role of corporate directors: Evidence from acquisition performance, Available at SSRN.

Che, Jiahua, and Yingyi Qian, 1998, Institutional environment, community government, and corporate governance: Understanding China's township-village enterprises, Journal of Law, Economics and Organization pp. 1-23.

Chen, Hui, Wei Luo, and Naomi S. Soderstrom, 2016, Career concerns and "unpaid" executives, Available at SSRN.

Chen, Jing, Mahmoud Ezzamel, and Ziming Cai, 2011, Managerial power theory, tournament theory, and executive pay in China, Journal of Corporate Finance 17, 1176-1199. 
Chou, Hsin-I, Huimin Chung, and Xiangkang Yin, 2013, Attendance of board meetings and company performance: Evidence from Taiwan, Journal of Banking 6 Finance 37, 4157-4171.

Claessens, Stijn, Simeon Djankov, Joseph PH Fan, and Larry HP Lang, 2002, Disentangling the incentive and entrenchment effects of large shareholdings, The Journal of Finance 57, 2741-2771.

Clarke, Donald C., 2006, Independent director in Chinese corporate governance, Delaware Journal of Corporate Law 31, 125-228.

Coles, Jeffrey L, and Chun-Keung Hoi, 2003, New evidence on the market for directors: Board membership and Pennsylvania senate bill 1310, The Journal of Finance 58, $197-230$.

Conyon, Martin J., and Lerong He, 2011, Executive compensation and corporate governance in China, Journal of Corporate Finance 17, 1158-1175.

Core, John E., Robert W. Holthausen, and David F. Larcker, 1999, Corporate governance, chief executive officer compensation, and firm performance, Journal of Financial Economics 51, 371-406.

Correia, Maria M., 2014, Political connections and sec enforcement, Journal of Accounting and Economics 57, 241-262.

CSRC, 2005, Notice of the china securities regulatory commission on promulgating the standards concerning the contents and formats of information disclosure by companies offering securities to the public no.2 - contents and formats of annual reports (2005 revision), issued by China Securities Regulatory Commission.

Cyert, Richard M., Sok-Hyon Kang, and Praveen Kumar, 2002, Corporate governance, takeovers, and top-management compensation: Theory and evidence, Management Science 48, 453-469.

Dahya, Jay, Yusuf Karbhari, and Jayson Zezong Xiao, 2002, The supervisory board in Chinese listed companies: Problems, causes, consequences and remedies, Asia Pacific Business Review 9, 118-137.

Dou, Ying, Sidharth Sahgal, and Emma Jincheng Zhang, 2015, Should independent directors have term limits? The role of experience in corporate governance, Financial Management 44, 583-621.

Dyl, Edward A., 1988, Corporate control and management compensation: Evidence on the agency problem, Managerial and Decision Economics 9, 21-25.

Edelen, Roger M., Ozgur S. Ince, and Gregory B. Kadlec, 2016, Institutional investors and stock return anomalies, Journal of Financial Economics 119, 472-488.

Ertugrul, Mine, and Shantaram Hegde, 2008, Board compensation practices and agency costs of debt, Journal of Corporate Finance 14, 512-531. 
Fagenson, Ellen A., and Janice J. Jackson, 1993, The status of women managers in the United States, International Studies of Management and Organization 23, 93-112.

Fama, Eugene F., and Michael C. Jensen, 1983, Separation of ownership and control, The Journal of Law and Economics 26, 301-325.

Fich, Eliezer M., and Anil Shivdasani, 2007, Financial fraud, director reputation, and shareholder wealth, Journal of Financial Economics 86, 306-336.

Field, Laura, Michelle Lowry, and Anahit Mkrtchyan, 2013, Are busy boards detrimental?, Journal of Financial Economics 109, 63-82.

Firth, Michael, Peter MY Fung, and Oliver M. Rui, 2007, How ownership and corporate governance influence chief executive pay in China's listed firms, Journal of Business Research 60, 776-785.

Gilson, Stuart C., 1990, Bankruptcy, boards, banks, and blockholders: Evidence on changes in corporate ownership and control when firms default, Journal of Financial Economics 27, 355-387.

Hermalin, Benjamin E., and Michael S. Weisbach, 2003, Boards of directors as an endogenously determined institution: A survey of the economic literature, Economic Policy Review pp. 7-26.

Houston, Joel F., Jiang Liangliang, Lin Chen, and Yue Ma, 2014, Political connections and the cost of bank loans, Journal of Accounting Research 52, 193-243.

Jensen, Michael C., 1986, Agency costs of free cash flow, corporate finance, and takeovers, The American Economic Review 76, 323-329.

, 1993, The modern industrial revolution, exit, and the failure of internal control systems, The Journal of Finance 48, 831-880.

Jiang, Fuxiu, and Kenneth A. Kim, 2015, Corporate governance in China: A modern perspective, Journal of Corporate Finance 32, 190-216.

Jiang, Guohua, Charles MC Lee, and Heng Yue, 2010, Tunneling through intercorporate loans: The China experience, Journal of Financial Economics 98, 1-20.

Kaplan, Steven N., and David Reishus, 1990, Outside directorships and corporate performance, Journal of Financial Economics 27, 389-410.

Kornai, Janos, 1980, Economics of shortage (Amsterdam (Netherlands) North-Holland).

Liang, Hao, Luc Renneboog, and Sunny Li Sun, 2015, The political determinants of executive compensation: Evidence from an emerging economy, Emerging Markets Review $25,69-91$.

Liu, Qiao, and Zhou Joe Lu, 2007, Corporate governance and earnings management in the Chinese listed companies: A tunneling perspective, Journal of Corporate Finance $13,881-906$. 
Lo, Agnes WY, Raymond MK Wong, and Michael Firth, 2010, Can corporate governance deter management from manipulating earnings? evidence from related-party sales transactions in China, Journal of Corporate Finance 16, 225-235.

Munisi, Gibson Hosea, and Roy Mersland, 2016, Ownership, board compensation and company performance in sub-Saharan African countries, Journal of Emerging Market Finance 15, 191-224.

Pinto, Marcos Barbosa, and Ricardo Pereira Câmara Leal, 2013, Ownership concentration, top management and board compensation, Revista de Administração Contemporânea 17, 304-324.

Porta, Rafael, Florencio Lopez-de Silanes, and Andrei Shleifer, 1999, Corporate ownership around the world, The Journal of Finance 54, 471-517.

Shleifer, Andrei, and Robert W. Vishny, 1986, Large shareholders and corporate control, Journal of Political Economy 94, 461-488.

— , 1997, A survey of corporate governance, The Journal of Finance 52, 737-783.

Tam, O. K., 1999, The development of corporate governance in China (Edward Elgar Publishers: Chaltenhamn, UK).

Tian, Lihui, 2001, State shareholding and the value of China's firms, in EFA 2001 Barcelona Meetings.

Yeh, Yin-Hua, and Tracie Woidtke, 2005, Commitment or entrenchment?: Controlling shareholders and board composition, Journal of Banking \& Finance 29, 1857-1885.

Zelechowski, Deborah Dahlen, and Diana Bilimoria, 2004, Characteristics of women and men corporate inside directors in the US, Corporate Governance: An International Review 12, 337-342. 


\subsection{Tables}

Table 1.1: Summary statistics

This table provides the summary statistics for all variables. Table 1.9 provides all variable definitions. Panel A provides the summary statistics for independent director. Panel B provides the summary statistics for non-independent director. Panel C provides the summary statistics for board characteristics in firm-year. Panel D provides the summary statistics for firm characteristics in year. All monetary terms are denominated in Chinese Yuan (CNY).

\begin{tabular}{|c|c|c|c|c|c|c|}
\hline & Obs & Mean & SD & 25 th & Median & 75 th \\
\hline \multicolumn{7}{|l|}{ Panel A. Independent director characteristics } \\
\hline $\operatorname{Unpaid}(0 / 1)_{t}$ & 60,318 & 0.06 & 0.24 & 0 & 0 & 0 \\
\hline Director compensation (Thousands $C N Y)_{t}$ & 60,318 & 57.65 & 53.48 & 34.08 & 50 & 70 \\
\hline $\operatorname{Woman}(0 / 1)$ & 60,318 & 0.15 & 0.35 & 0 & 0 & 0 \\
\hline Busy director $(0 / 1)_{t}$ & 60,318 & 0.31 & 0.46 & 0 & 0 & 1 \\
\hline Number of directorships ${ }_{t}$ & 60,318 & 2.21 & 1.64 & 1 & 2 & 3 \\
\hline Tenure $_{t}$ & 60,318 & 6.11 & 3.67 & 3 & 6 & 8 \\
\hline $\mathrm{Age}_{t}$ & 60,318 & 53.15 & 9.61 & 46 & 51 & 60 \\
\hline New director $(0 / 1)_{t}$ & 60,318 & 0.17 & 0.37 & 0 & 0 & 0 \\
\hline Political background $(0 / 1)_{t}$ & 47,214 & 0.39 & 0.49 & 0 & 0 & 1 \\
\hline \multicolumn{7}{|l|}{ Panel B. Non-independent director characteristics } \\
\hline $\operatorname{Unpaid}(0 / 1)_{t}$ & 95,173 & 0.36 & 0.48 & 0 & 0 & 1 \\
\hline Director compensation(Thousands $C N Y)_{t}$ & 95,173 & 288.16 & 537.20 & 0 & 120 & 400 \\
\hline $\operatorname{Woman}(0 / 1)$ & 95,173 & 0.11 & 0.31 & 0 & 0 & 0 \\
\hline Related director $(0 / 1)_{t}$ & 95,173 & 0.41 & 0.49 & 0 & 0 & 1 \\
\hline Tenure $_{t}$ & 95,173 & 5.90 & 3.91 & 3 & 5 & 8 \\
\hline $\mathrm{Age}_{t}$ & 95,173 & 49.05 & 7.64 & 44 & 49 & 54 \\
\hline New director $(0 / 1)_{t}$ & 95,173 & 0.13 & 0.33 & 0 & 0 & 0 \\
\hline Share ownership(Millions Shares) $)_{t}$ & 95,160 & 5.47 & 34.36 & 0 & 0 & 0.03 \\
\hline \multicolumn{7}{|l|}{ Panel C. Board characteristics (by firm-year) } \\
\hline Board size $_{t}$ & 16,543 & 8.95 & 1.91 & 8 & 9 & 9 \\
\hline Board composition $(\operatorname{Ind} \%)_{t}$ & 16,543 & 0.37 & 0.05 & 0.33 & 0.33 & 0.40 \\
\hline Dualityt & 16,543 & 0.21 & 0.41 & 0 & 0 & 0 \\
\hline Meeting frequency $(I d)_{t}$ & 16,542 & 8.38 & 3.72 & 6 & 8 & 10 \\
\hline Meeting frequency $(\text { Firm })_{t}$ & 16,529 & 9.49 & 3.85 & 7 & 9 & 11 \\
\hline \multicolumn{7}{|l|}{ Panel D. Firm characteristics (by year) } \\
\hline State-owned $(0 / 1)_{t}$ & 16,543 & 0.48 & 0.50 & 0 & 0 & 1 \\
\hline Ownership concentration $_{t}$ & 16,543 & 0.33 & 0.17 & 0.20 & 0.32 & 0.45 \\
\hline Excess control right $t_{t}$ & 16,543 & 0.06 & 0.08 & 0 & 0 & 0.10 \\
\hline Cash holdings $_{t}$ & 16,543 & 0.16 & 0.15 & 0.06 & 0.11 & 0.21 \\
\hline Total assets $(\text { Billions } C N Y)_{t}$ & 16,543 & 42.09 & 57.88 & 1.29 & 2.74 & 6.7 \\
\hline Book leverage $_{t}$ & 16,543 & 0.53 & 1.59 & 0.30 & 0.47 & 0.64 \\
\hline$R O A_{t}$ & 16,543 & 0.03 & 0.69 & 0.01 & 0.03 & 0.06 \\
\hline Stock volatilityt & 16,491 & 0.15 & 0.11 & 0.10 & 0.13 & 0.18 \\
\hline
\end{tabular}


Table 1.2: Cross-correlations

This table provides the correlation matrix of the key variables. Panel A provides the correlation matrix for non-independent director. Panel B provides the correlation matrix for independent director. Table 1.9 provides all variable definitions. All monetary terms are denominated in Chinese Yuan (CNY). Superscripts $*, * *$ and $* * *$ denote significance at the $10 \%, 5 \%$ and $1 \%$ levels, respectively.

\begin{tabular}{|c|c|c|c|c|c|c|c|c|c|}
\hline Panel A. Non-independent director & (1) & (2) & (3) & $(4)$ & (5) & $(6)$ & $(7)$ & $(8)$ & $(9)$ \\
\hline (1) $\operatorname{Unpaid}(0 / 1)_{t}$ & 1 & & & & & & & & \\
\hline (2) Director compensation ${ }_{t}$ & $-0.359^{* *}$ & 1 & & & & & & & \\
\hline (3) $\operatorname{Woman}(0 / 1)$ & $-0.0121^{* *}$ & $-0.0205^{* *}$ & 1 & & & & & & \\
\hline (4) Related director $(0 / 1)_{t}$ & $0.396^{* *}$ & $-0.148^{* *}$ & $-0.0177^{* *}$ & 1 & & & & & \\
\hline (5) Tenuret $_{t}$ & $-0.0455^{* *}$ & $0.0995^{* *}$ & $-0.0381^{* *}$ & $0.0155^{* *}$ & 1 & & & & \\
\hline (6) State-owned $(0 / 1)_{t}$ & $0.223^{* *}$ & $-0.0798^{* *}$ & $-0.0807^{* *}$ & $0.186^{* *}$ & $0.136^{* *}$ & 1 & & & \\
\hline (7) Ownership concentration con $_{1}$ & $0.0354^{* *}$ & 0.00133 & 0.00195 & 0.00600 & $-0.112^{* *}$ & $0.192^{* *}$ & 1 & & \\
\hline (8) Excess control right $t_{t}$ & $0.0426^{* *}$ & $-0.0104^{* *}$ & $-0.0109 * *$ & $0.119^{* *}$ & $0.0302^{* *}$ & $-0.163^{* *}$ & $-0.407^{* *}$ & 1 & \\
\hline (9) New director $(0 / 1)_{t}$ & $0.0529 * *$ & $-0.0560^{* *}$ & $0.0167^{* *}$ & $-0.0204^{* *}$ & $-0.329 * *$ & $-0.0619^{* *}$ & $0.0716^{* *}$ & $-0.0218^{* *}$ & 1 \\
\hline Panel B. Independent director & $(1)$ & $(2)$ & $(3)$ & $(4)$ & $(5)$ & $(6)$ & $(7)$ & $(8)$ & $(9)$ \\
\hline (1) $\operatorname{Unpaid}(0 / 1)_{t}$ & 1 & & & & & & & & \\
\hline (2) Director compensation con $_{t}$ & $-0.281^{* *}$ & 1 & & & & & & & \\
\hline (3) $\operatorname{Woman}(0 / 1)$ & 0.00753 & $-0.0243^{* *}$ & 1 & & & & & & \\
\hline (4) Busy director $(0 / 1)_{t}$ & $-0.0216^{* *}$ & $0.0343^{* *}$ & $-0.0358^{* *}$ & 1 & & & & & \\
\hline (5) Tenuret & $-0.0293^{* *}$ & $0.0659 * *$ & $-0.0302 * *$ & $0.441^{* *}$ & 1 & & & & \\
\hline (6) State-owned $(0 / 1)_{t}$ & 0.00665 & $0.0400^{* *}$ & $-0.0324^{* *}$ & 0.000626 & $0.0393^{* *}$ & 1 & & & \\
\hline (7) Ownership concentration ${ }_{t}$ & $-0.0165^{* *}$ & $0.0820^{* *}$ & $-0.0258^{* *}$ & $0.0230^{* *}$ & $0.00998^{*}$ & $0.182^{* *}$ & 1 & & \\
\hline (8) Excess control right $t_{t}$ & -0.00269 & $-0.0342^{* *}$ & 0.00216 & -0.00740 & $-0.0161^{* *}$ & $-0.160^{* *}$ & $-0.404^{* *}$ & 1 & \\
\hline (9) New director $(0 / 1)_{t}$ & $0.224^{* *}$ & $-0.177^{* *}$ & $0.0147^{* *}$ & $-0.0332^{* *}$ & $-0.222^{* *}$ & $-0.0875^{* *}$ & $0.0612^{* *}$ & $-0.0191^{* *}$ & 1 \\
\hline
\end{tabular}


Table 1.3: Testing hypothesis 1 and 2

This table provides the baseline regression results. The regression on both propensity being unpaid and level of compensation are reported with coefficients. Table 1.9 provides all variable definitions. The regressions control for time and industry effects. All monetary terms are denominated in Chinese Yuan (CNY). Superscripts * ** and *** denote significance at the $10 \%, 5 \%$ and $1 \%$ levels, respectively.

\begin{tabular}{|c|c|c|c|c|}
\hline \multirow[b]{2}{*}{ Explanatory variables } & \multicolumn{2}{|c|}{ Independent directors } & \multicolumn{2}{|c|}{ Non-independent directors } \\
\hline & $\begin{array}{l}\text { Unpaid }(0 / 1) \\
(1)\end{array}$ & $\begin{array}{c}\text { Ln(Compensation) } \\
(2)\end{array}$ & $\begin{array}{c}\text { Unpaid }(0 / 1) \\
(3)\end{array}$ & $\begin{array}{c}\text { Ln(Compensation) } \\
(4)\end{array}$ \\
\hline $\operatorname{Woman}(0 / 1)$ & $\begin{array}{c}0.0642 \\
(0.0489)\end{array}$ & $\begin{array}{l}-0.00971 \\
(0.00630)\end{array}$ & $\begin{array}{c}-0.127^{* * *} \\
(0.0268)\end{array}$ & $\begin{array}{c}0.0121 \\
(0.0130)\end{array}$ \\
\hline Busy director $(0 / 1)_{t}$ & $\begin{array}{l}-0.131^{* * *} \\
(0.0437)\end{array}$ & $\begin{array}{c}0.0274^{* * *} \\
(0.00513)\end{array}$ & & \\
\hline Tenure $_{t}$ & $\begin{array}{c}-0.0400^{* * *} \\
(0.00535)\end{array}$ & $\begin{array}{c}0.00415 * * * \\
(0.000681)\end{array}$ & $\begin{array}{c}-0.0330 * * * \\
(0.00211)\end{array}$ & $\begin{array}{c}0.0196 * * * \\
(0.00113)\end{array}$ \\
\hline State-owned $(0 / 1)_{t}$ & $\begin{array}{l}0.0786^{*} \\
(0.0416)\end{array}$ & $\begin{array}{c}-0.0823^{* * *} \\
(0.00522)\end{array}$ & $\begin{array}{l}0.660 * * * \\
(0.0200)\end{array}$ & $\begin{array}{c}-0.110^{* * *} \\
(0.0112)\end{array}$ \\
\hline Ownership concentration $_{t}$ & $\begin{array}{c}-0.429^{* * *} \\
(0.120)\end{array}$ & $\begin{array}{l}0.0339 * * \\
(0.0152)\end{array}$ & $\begin{array}{l}0.604^{* * *} * \\
(0.0563)\end{array}$ & $\begin{array}{l}0.353^{* * *} \\
(0.0292)\end{array}$ \\
\hline Excess control right $t_{t}$ & $\begin{array}{l}-0.109 \\
(0.239)\end{array}$ & $\begin{array}{l}-0.0100 \\
(0.0300)\end{array}$ & $\begin{array}{c}0.882^{* * *} \\
(0.114)\end{array}$ & $\begin{array}{l}0.924^{* * * *} \\
(0.0623)\end{array}$ \\
\hline Related director $(0 / 1)_{t}$ & & & $\begin{array}{l}1.764^{* * *} \\
(0.0167)\end{array}$ & $\begin{array}{c}-0.371^{* * *} \\
(0.0108)\end{array}$ \\
\hline$C E O / C O B(0 / 1)_{t}$ & & & $\begin{array}{l}-1.476^{* * *} \\
(0.0201)\end{array}$ & $\begin{array}{l}0.773^{* * *} \\
(0.00870)\end{array}$ \\
\hline$A g e_{t}$ & $\begin{array}{l}-0.0245 \\
(0.0180)\end{array}$ & $\begin{array}{c}0.0158 * * * \\
(0.00244)\end{array}$ & $\begin{array}{c}0.0105 \\
(0.00947)\end{array}$ & $\begin{array}{c}0.0787^{* * *} \\
(0.00466)\end{array}$ \\
\hline$A g e_{t}^{2}$ & $\begin{array}{l}0.000267 * \\
(0.000161)\end{array}$ & $\begin{array}{c}-0.000115^{* * *} \\
(2.20 \mathrm{e}-05)\end{array}$ & $\begin{array}{c}0.000122 \\
(9.35 \mathrm{e}-05)\end{array}$ & $\begin{array}{c}-0.000815^{* * *} \\
(4.69 \mathrm{e}-05)\end{array}$ \\
\hline Ln(Share ownership +1$)_{t}$ & & & $\begin{array}{c}-0.0808^{* * *} \\
(0.00167)\end{array}$ & $\begin{array}{l}0.0285^{* * * *} \\
(0.000628)\end{array}$ \\
\hline $\operatorname{Ln}(C E O \text { compensation }+1)_{t}$ & $\begin{array}{c}-0.0307^{* * *} \\
(0.00373)\end{array}$ & $\begin{array}{c}0.00577 * * * \\
(0.000541)\end{array}$ & $\begin{array}{c}-0.0492^{* * *} \\
(0.00197)\end{array}$ & $\begin{array}{c}0.0294^{* * *} \\
(0.00132)\end{array}$ \\
\hline Meeting frequency $(I d)_{t}$ & $\begin{array}{l}-0.283^{* * *} \\
(0.00857)\end{array}$ & $\begin{array}{l}0.0468 * * * \\
(0.000820)\end{array}$ & & \\
\hline Meeting frequency $(\text { Firm })_{t}$ & & & $\begin{array}{c}-0.00646 * * * \\
(0.00222)\end{array}$ & $\begin{array}{l}-0.00135 \\
(0.00121)\end{array}$ \\
\hline Duality $_{t}$ & $\begin{array}{l}0.0786^{*} \\
(0.0461)\end{array}$ & $\begin{array}{c}0.00807 \\
(0.00566)\end{array}$ & $\begin{array}{c}-0.199 * * * \\
(0.0229)\end{array}$ & $\begin{array}{c}0.0745^{* * *} \\
(0.0100)\end{array}$ \\
\hline Board sizet & $\begin{array}{r}-0.00902 \\
(0.0109)\end{array}$ & $\begin{array}{c}0.0146^{* * *} \\
(0.00141)\end{array}$ & $\begin{array}{l}-0.00173 \\
(0.00478)\end{array}$ & $\begin{array}{c}0.00790 * * * \\
(0.00271)\end{array}$ \\
\hline Board composition $(\text { Ind } \%)_{t}$ & $\begin{array}{c}-0.733^{* *} \\
(0.361)\end{array}$ & $\begin{array}{c}0.475^{* * *} \\
(0.0419)\end{array}$ & $\begin{array}{c}-0.432^{* *} \\
(0.180)\end{array}$ & $\begin{array}{l}0.386 * * * \\
(0.0870)\end{array}$ \\
\hline Ln(Total Assets $)_{t}$ & $\begin{array}{c}0.0681^{* * *} \\
(0.0149)\end{array}$ & $\begin{array}{l}0.124^{* * *} \\
(0.00220)\end{array}$ & $\begin{array}{c}-0.0405^{* * *} \\
(0.00760)\end{array}$ & $\begin{array}{l}0.270 * * * \\
(0.00421)\end{array}$ \\
\hline Cash holdingst & $\begin{array}{c}-0.744^{* * *} \\
(0.145)\end{array}$ & $\begin{array}{l}0.355^{* * *} \\
(0.0174)\end{array}$ & $\begin{array}{c}0.333^{* * *} \\
(0.0622)\end{array}$ & $\begin{array}{l}0.533 * * * \\
(0.0311)\end{array}$ \\
\hline Book leverage $_{t}$ & $\begin{array}{l}0.0151^{* *} \\
(0.00728)\end{array}$ & $\begin{array}{l}0.00430 * \\
(0.00251)\end{array}$ & $\begin{array}{c}0.0466^{* * *} \\
(0.0117)\end{array}$ & $\begin{array}{c}-0.0109 \\
(0.00678)\end{array}$ \\
\hline$R O A_{t-1}$ & $\begin{array}{c}-0.000148^{* * *} \\
(5.01 \mathrm{e}-05)\end{array}$ & $\begin{array}{c}1.93 \mathrm{e}-05 \\
(2.67 \mathrm{e}-05)\end{array}$ & $\begin{array}{l}0.00119 * * \\
(0.000530)\end{array}$ & $\begin{array}{c}-0.000728^{*} \\
(0.000375)\end{array}$ \\
\hline Stock volatilityt-1 & $\begin{array}{l}-0.188 \\
(0.116)\end{array}$ & $\begin{array}{l}-0.0110 \\
(0.0141)\end{array}$ & $\begin{array}{c}0.233^{* * *} \\
(0.0553)\end{array}$ & $\begin{array}{c}-0.00928 \\
(0.0410)\end{array}$ \\
\hline Year effects & Yes & Yes & Yes & Yes \\
\hline Industry effects & Yes & Yes & Yes & Yes \\
\hline Director effects & No & No & No & No \\
\hline Observations & 60,231 & 56,633 & 95,160 & 61,305 \\
\hline
\end{tabular}


Table 1.4: Estimated economic impact

We report the estimated effect of a one standard deviation increase (from the mean value) for continuous variables and one unit increase (from zero to one) for dummy variables on both the probability a director is unpaid and the level of director

compensation. Table 1.9 provides all variable definitions. Columns 3 and 4 provide the predicted signs by hypothesis. Columns 5 and 6 provide the percentage changes of both the propensity to be unpaid and the level of director compensation from the mean value for independent directors. Columns 7 and 8 provide the percentage changes of both the propensity to be unpaid and the level of director compensation from the mean value for non-independent directors. n.s. denotes not statistically significant. n.a. denotes not applicable. Superscripts ${ }^{*},{ }^{*}$ and ${ }^{* * *}$ denote significance at the $10 \%, 5 \%$ and $1 \%$ levels, respectively.

\begin{tabular}{llllllll}
\hline & & \multicolumn{3}{c}{} & \multicolumn{2}{c}{ Independent } & \multicolumn{2}{c}{ Non-independent } \\
Hypothesis & Variable of Interest & Unpaid & Level & Unpaid & Level & Unpaid & Level \\
\hline H1a & Woman $(0 / 1)$ & + & - & n.s. & n.s. & $-4.39^{* * *}$ & n.s. \\
H1b & Busy director $(0 / 1)$ & - & + & $-11.18^{* * *}$ & $2.78^{* * *}$ & n.a. & n.a. \\
H1c & Tenure & - & + & $-13.53^{* * *}$ & $1.62^{* * *}$ & $-11.68^{* * *}$ & $13.94^{* * *}$ \\
H2a & State-owned $(0 / 1)$ & + & - & $6.61^{*}$ & $-7.92^{* * *}$ & $31.20^{* * *}$ & $-11.57^{* * *}$ \\
H2b & Ownership Concentration & + & - & $-6.46^{* * *}$ & $0.59^{* *}$ & $4.36^{* * *}$ & $6.78^{* * *}$ \\
H2c & Excess control rights & + & - & n.s. & n.s. & $3.29^{* * *}$ & $7.72^{* * *}$ \\
H2d & Related director $(0 / 1)$ & + & - & n.a. & n.a. & $89.95^{* * *}$ & $-30.65^{* * *}$ \\
\hline
\end{tabular}


Table 1.5: Robustness: Tenure and new director

This table provides the regression results for Tenure, Tenure $e_{t}^{2}$ and New director $(0 / 1)_{t}$. Panel A provides the regression results on the propensity being unpaid. Panel B provides the regression results on the level of compensation. Columns (1)-(4) provide the regression results of independent directors. Columns (5)-(8) provide the regression results of non-independent directors. The regression on both propensity being unpaid and level of compensation are reported with coefficients. Table 1.9 provides all variable definitions. We include all other variables from the baseline regressions (both variables of interest and control variables) as our control variables here. The regressions control for time and industry effects. All monetary terms are denominated in Chinese Yuan (CNY). Superscripts *,** and ${ }^{* * *}$ denote significance at the $10 \%, 5 \%$ and $1 \%$ levels, respectively.

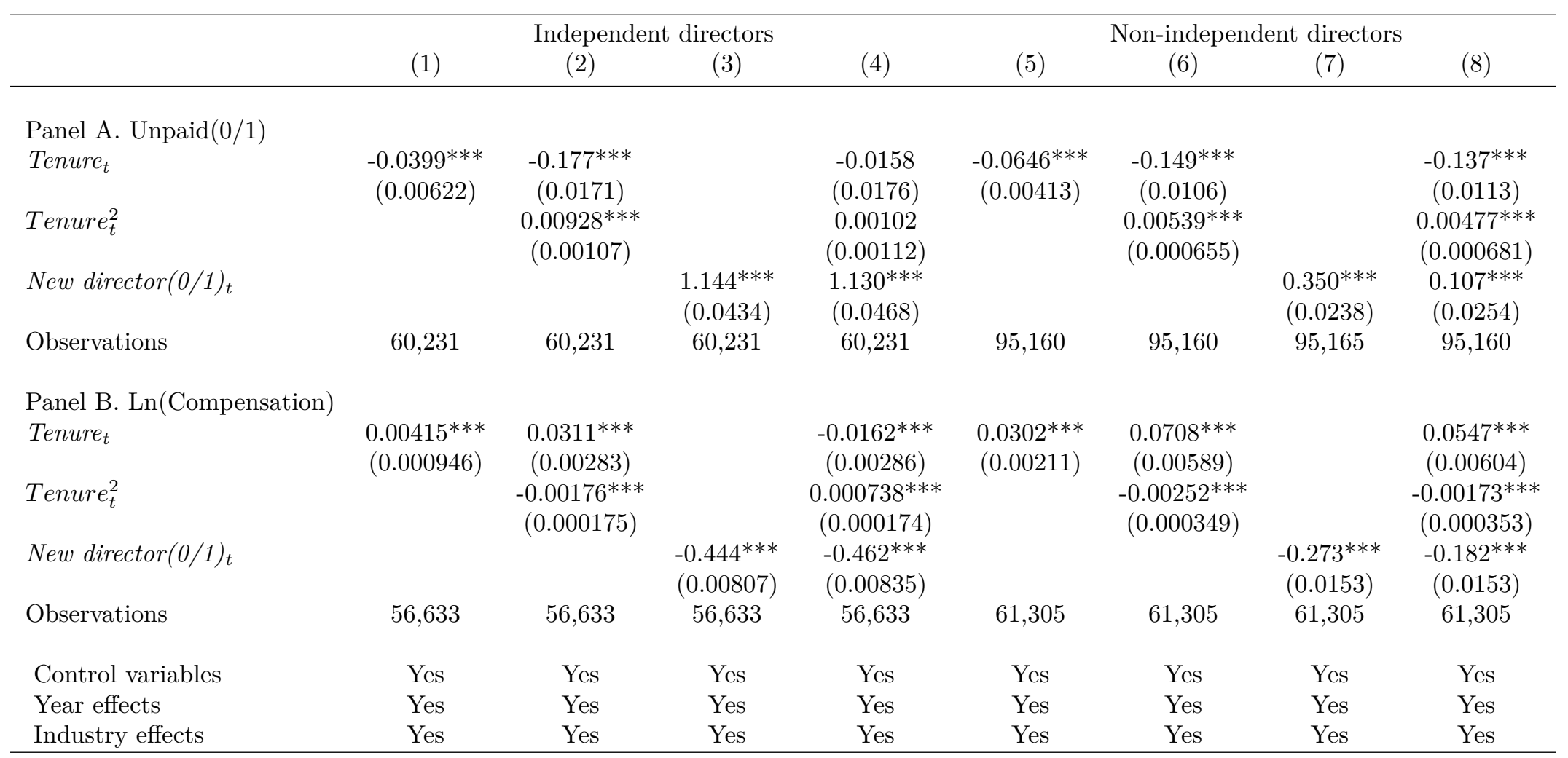


Table 1.6: Robustness: Political connections

This table provides the regression results of Political background $(0 / 1)_{t}$ on director compensation. We separate the whole sample into two sub-samples: 1) directors working in state-owned firms; 2) directors working in non-state-owned firms. Panel A provides the regression results of state-owned firms. Panel B provides the regression results of non-state-owned firms. For each sub-samples, we provides the regression results of independent directors and non-independent directors. The regressions on both propensity being unpaid and level of compensation are reported with coefficients. Table 1.9 provides all variable definitions. We include all other variables from the baseline regressions (both variables of interest and control variables) as our control variables here. The regressions control for time and industry effects. All monetary terms are denominated in Chinese Yuan (CNY). Superscripts *, ** and *** denote significance at the $10 \%, 5 \%$ and $1 \%$ levels, respectively.

\begin{tabular}{|c|c|c|c|c|}
\hline & \multicolumn{2}{|c|}{ Independent directors } & \multicolumn{2}{|c|}{ Non-independent directors } \\
\hline & $\begin{array}{c}\text { Unpaid }(0 / 1) \\
(1)\end{array}$ & $\begin{array}{c}\operatorname{Ln}(\text { Compensation }) \\
(2)\end{array}$ & $\begin{array}{c}\text { Unpaid }(0 / 1) \\
(3)\end{array}$ & $\begin{array}{c}\operatorname{Ln}(\text { Compensation }) \\
(4)\end{array}$ \\
\hline \multicolumn{5}{|l|}{ Panel A. State-owned firms } \\
\hline Political background $(0 / 1)_{t}$ & $\begin{array}{c}0.00727^{* *} \\
(0.00322)\end{array}$ & $\begin{array}{c}0.0127^{*} \\
(0.00769)\end{array}$ & $\begin{array}{c}-0.00262 \\
(0.00556)\end{array}$ & $\begin{array}{c}-0.0280 \\
(0.0224)\end{array}$ \\
\hline Observations & 23,676 & 22,204 & 37,464 & 19,812 \\
\hline \multicolumn{5}{|c|}{ Panel B. Non-state-owned firms } \\
\hline Political background $(0 / 1)_{t}$ & $\begin{array}{c}0.00406 \\
(0.00313)\end{array}$ & $\begin{array}{c}0.0140^{*} \\
(0.00731)\end{array}$ & $\begin{array}{c}0.00627 \\
(0.00535)\end{array}$ & $\begin{array}{l}0.0310^{*} \\
(0.0163)\end{array}$ \\
\hline Observations & 23,538 & 22,241 & 36,976 & 27,955 \\
\hline Control variables & Yes & Yes & Yes & Yes \\
\hline Year effects & Yes & Yes & Yes & Yes \\
\hline Industry effects & Yes & Yes & Yes & Yes \\
\hline
\end{tabular}


Table 1.7: Robustness: Director effects

This table provides the mixed regression results with director effects. The regressions on both propensity being unpaid and level of compensation are reported with coefficients. Table 1.9 provides all variable definitions. The regressions control for time, industry and director effects. All monetary terms are denominated in Chinese Yuan (CNY). Standard errors are adjusted and clustered on the director identifier. Superscripts ${ }^{*}, * *$ and ${ }^{* * *}$ denote significance at the $10 \%, 5 \%$ and $1 \%$ levels, respectively.

\begin{tabular}{|c|c|c|c|c|}
\hline \multirow[b]{2}{*}{ Explanatory variables } & \multicolumn{2}{|c|}{ Independent directors } & \multicolumn{2}{|c|}{ Non-independent directors } \\
\hline & $\begin{array}{c}\text { Unpaid(0/1) } \\
(1)\end{array}$ & $\begin{array}{c}\operatorname{Ln}(\text { Compensation }) \\
(2)\end{array}$ & $\begin{array}{c}\text { Unpaid }(0 / 1) \\
(3)\end{array}$ & $\operatorname{Ln}($ Compensation $)$ \\
\hline $\operatorname{Woman}(0 / 1)$ & $\begin{array}{c}0.105 \\
(0.0796)\end{array}$ & $\begin{array}{c}-0.0117 \\
(0.00955)\end{array}$ & $\begin{array}{c}-0.189^{* * *} \\
(0.0562)\end{array}$ & $\begin{array}{l}-0.0391 \\
(0.0244)\end{array}$ \\
\hline Busy director $(0 / 1)_{t}$ & $\begin{array}{c}-0.188^{* * *} \\
(0.0654)\end{array}$ & $\begin{array}{c}0.0281^{* * *} \\
(0.00607)\end{array}$ & & \\
\hline Tenure $_{t}$ & $\begin{array}{c}-0.0530 * * * \\
(0.00825)\end{array}$ & $\begin{array}{r}0.00614^{* * *} \\
(0.000914)\end{array}$ & $\begin{array}{l}-0.211^{* * *} \\
(0.00830)\end{array}$ & $\begin{array}{l}0.0139 * * * \\
(0.00191)\end{array}$ \\
\hline State-owned $(0 / 1)_{t}$ & $\begin{array}{c}0.0892 \\
(0.0669)\end{array}$ & $\begin{array}{c}-0.0589 * * * \\
(0.00759)\end{array}$ & $\begin{array}{c}4.567 * * * \\
(0.149)\end{array}$ & $\begin{array}{c}-0.130 * * * \\
(0.0191)\end{array}$ \\
\hline Ownership concentration $_{t}$ & $\begin{array}{c}-0.622^{* * *} \\
(0.192)\end{array}$ & $\begin{array}{l}0.0503^{* *} \\
(0.0214)\end{array}$ & $\begin{array}{c}0.783^{* * *} \\
(0.147)\end{array}$ & $\begin{array}{l}0.303^{* * *} \\
(0.0442)\end{array}$ \\
\hline Excess control right $t_{t}$ & $\begin{array}{l}-0.134 \\
(0.383)\end{array}$ & $\begin{array}{l}0.0241 \\
(0.0406)\end{array}$ & $\begin{array}{c}4.543 * * * \\
(0.334)\end{array}$ & $\begin{array}{c}0.337 * * * \\
(0.0882)\end{array}$ \\
\hline Related director $(0 / 1)_{t}$ & & & $\begin{array}{c}4.465^{* * *} \\
(0.130)\end{array}$ & $\begin{array}{c}-0.0763^{* * *} \\
(0.0137)\end{array}$ \\
\hline$C E O / C O B(0 / 1)_{t}$ & & & $\begin{array}{c}-5.142^{* * *} \\
(0.164)\end{array}$ & $\begin{array}{c}0.488^{* * *} \\
(0.0142)\end{array}$ \\
\hline $\mathrm{Age}_{t}$ & $\begin{array}{c}-0.0590^{*} \\
(0.0308)\end{array}$ & $\begin{array}{c}0.0239 * * * \\
(0.00351)\end{array}$ & $\begin{array}{c}0.105^{* * *} \\
(0.0240)\end{array}$ & $\begin{array}{l}0.0760 * * * \\
(0.00802)\end{array}$ \\
\hline$A g e_{t}^{2}$ & $\begin{array}{c}0.000559^{* *} \\
(0.000279)\end{array}$ & $\begin{array}{c}-0.000181^{* * *} \\
(3.16 \mathrm{e}-05)\end{array}$ & $\begin{array}{c}0.000307 \\
(0.000235)\end{array}$ & $\begin{array}{c}-0.000790^{* * *} \\
(8.11 \mathrm{e}-05)\end{array}$ \\
\hline Ln $(\text { Share ownership }+1)_{t}$ & & & $\begin{array}{c}-0.355^{* * *} \\
(0.0109)\end{array}$ & $\begin{array}{l}0.0229 * * * \\
(0.00106)\end{array}$ \\
\hline $\operatorname{Ln}(C E O \text { compensation }+1)_{t}$ & $\begin{array}{c}-0.0405^{* * *} \\
(0.00555)\end{array}$ & $\begin{array}{c}0.00345^{* * *} \\
(0.000544)\end{array}$ & $\begin{array}{c}-0.115^{* * *} \\
(0.00663)\end{array}$ & $\begin{array}{c}0.00987^{* * * *} \\
(0.00115)\end{array}$ \\
\hline Meeting frequency $(I d)_{t}$ & $\begin{array}{c}-0.375^{* * *} \\
(0.0134)\end{array}$ & $\begin{array}{l}0.0636^{* * *} \\
(0.00103)\end{array}$ & & \\
\hline Meeting frequency $(\text { Firm })_{t}$ & & & $\begin{array}{l}-0.00244 \\
(0.00628)\end{array}$ & $\begin{array}{c}-0.00468^{* * *} \\
(0.000992)\end{array}$ \\
\hline Dualityt $_{t}$ & $\begin{array}{c}0.0888 \\
(0.0703)\end{array}$ & $\begin{array}{c}0.00339 \\
(0.00674)\end{array}$ & $\begin{array}{c}-0.530 * * * \\
(0.0669)\end{array}$ & $\begin{array}{l}0.0150 \\
(0.0114)\end{array}$ \\
\hline Board size $_{t}$ & $\begin{array}{l}0.00432 \\
(0.0168)\end{array}$ & $\begin{array}{l}0.0181^{* * *} \\
(0.00188)\end{array}$ & $\begin{array}{l}0.00712 \\
(0.0138)\end{array}$ & $\begin{array}{l}0.0142^{* * * *} \\
(0.00372)\end{array}$ \\
\hline Board composition $(\text { Ind\% })_{t}$ & $\begin{array}{l}-0.537 \\
(0.548)\end{array}$ & $\begin{array}{l}0.493^{* * *} \\
(0.0524)\end{array}$ & $\begin{array}{c}0.408 \\
(0.532)\end{array}$ & $\begin{array}{c}0.107 \\
(0.0998)\end{array}$ \\
\hline Ln(Total Assets $)_{t}$ & $\begin{array}{c}0.103 * * * \\
(0.0251)\end{array}$ & $\begin{array}{c}0.0948 * * * \\
(0.00328)\end{array}$ & $\begin{array}{c}-0.221^{* * *} \\
(0.0218)\end{array}$ & $\begin{array}{l}0.208^{* * *} \\
(0.00723)\end{array}$ \\
\hline Cash holdingst & $\begin{array}{c}-0.998^{* * *} \\
(0.215)\end{array}$ & $\begin{array}{l}0.242^{* * *} \\
(0.0213)\end{array}$ & $\begin{array}{c}0.880^{* * *} \\
(0.183)\end{array}$ & $\begin{array}{l}0.273^{* * *} \\
(0.0294)\end{array}$ \\
\hline Book leverage $_{t}$ & $\begin{array}{l}0.0210^{* *} \\
(0.0107)\end{array}$ & $\begin{array}{c}0.00276 \\
(0.00243)\end{array}$ & $\begin{array}{c}0.159 * * * \\
(0.0259)\end{array}$ & $\begin{array}{c}-0.000540 \\
(0.00387)\end{array}$ \\
\hline$R O A_{t-1}$ & $\begin{array}{c}-0.000197^{* * *} \\
(5.79 \mathrm{e}-05)\end{array}$ & $\begin{array}{l}-1.96 \mathrm{e}-05 \\
(2.45 \mathrm{e}-05)\end{array}$ & $\begin{array}{c}0.00444^{* * *} \\
(0.00130)\end{array}$ & $\begin{array}{c}-0.000395 * * \\
(0.000157)\end{array}$ \\
\hline Stock volatilityt-1 & $\begin{array}{l}-0.388^{*} \\
(0.199)\end{array}$ & $\begin{array}{l}-0.00775 \\
(0.0116)\end{array}$ & $\begin{array}{c}0.566^{* * *} \\
(0.144)\end{array}$ & $\begin{array}{l}-0.0101 \\
(0.0198)\end{array}$ \\
\hline Year effects & Yes & Yes & Yes & Yes \\
\hline Industry effects & Yes & Yes & Yes & Yes \\
\hline Director effect & Yes & Yes & Yes & Yes \\
\hline Observations & 60,231 & 56,633 & 95,160 & 61,305 \\
\hline
\end{tabular}


Table 1.8: Robustness: Ownership concentration (state-owned versus non-state-owned)

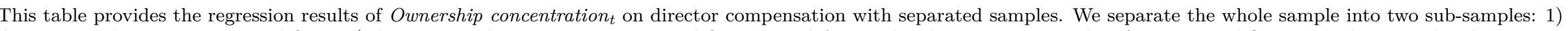
directors working in state-owned firms; 2) directors working in non-state-owned firms. Panel A provides the regression results of state-owned firms. Panel B provides the regression results of non-state-owned firms. For each sub-samples, we provides the regression results of independent directors, non-executive non-independent directors, CEO and COB. The regressions on both propensity being unpaid and level of compensation are reported with coefficients. Table 1.9 provides all variable definitions. We include all other variables from the baseline regressions (both variables of interest and control variables) as our control variables here. The regressions control for time and industry effects. All monetary terms are denominated in Chinese Yuan (CNY). Superscripts *, ** and ${ }^{* * *}$ denote significance at the $10 \%, 5 \%$ and $1 \%$ levels, respectively.

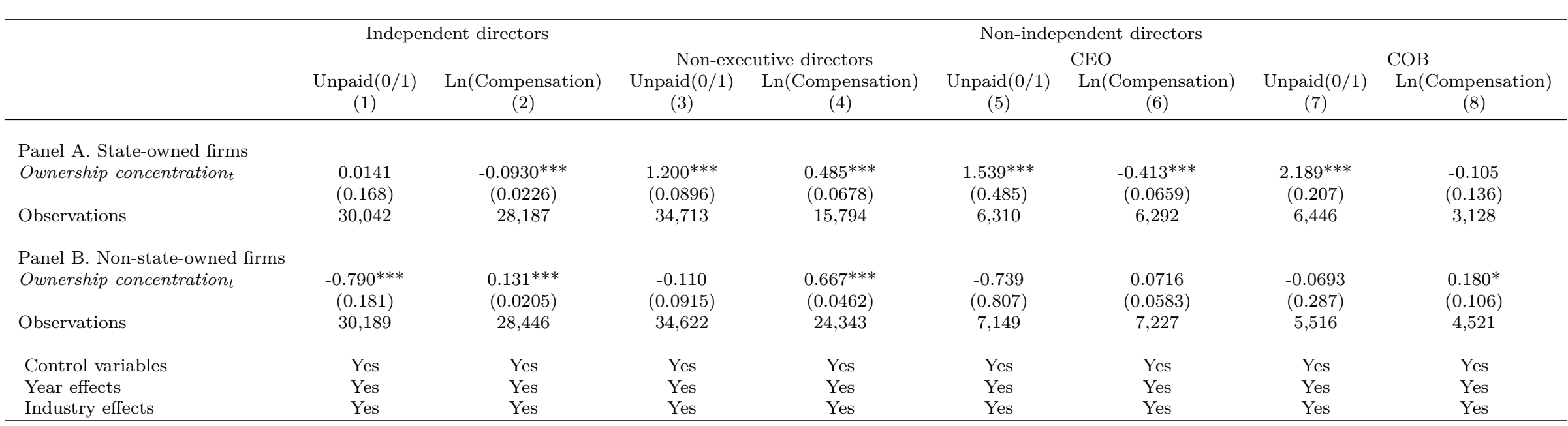




\subsection{Appendix}

Table 1.9: Variable definitions

Variable Description

\section{Panel A. Dependent variables}

Ln(Compensation) $t$

The logarithm of compensation that a board of director receives from a firm in the year $t$.

$\operatorname{Unpaid}(0 / 1)_{t}$

The dummy variable equals to 1 if a board of director is unpaid in a firm in the year $t$ and 0 otherwise.

\section{Panel B. Variables of interest}

$\operatorname{Woman}(0 / 1)$

The dummy variable equals to 1 if a board of director is female and 0 otherwise.

The dummy variable equals to 1 if an independent director holds more than two directorships in the year $t$ and 0 otherwise.

Related director $(0 / 1)_{t}$

Tenure $_{t}$

State-owned $(0 / 1)_{t}$

Ownership concentration

The dummy variable equals to 1 if a board of director holds a position in the controlling firm in the year $t$ and 0 otherwise.

The number of year that a board of director serves as a board of director in the year $t$.

The dummy variable equals to 1 if the firm is state-owned in the year $t$ and 0 otherwise.

The proportion of ownership(cash-flow rights) held by the ultimate controlling shareholders in the year $t$.

Excess control right

The percentage difference between controlling rights and cash-flow rights of the ultimate controlling shareholders in the year $t$. 
Table 1.9: Variable definitions

Variable

New director $(0 / 1)_{t}$

Political background $(0 / 1)_{t}$

\section{Panel C. Control variables} $C E O / C O B(0 / 1)_{t}$

Age $_{t}$

$A g e_{t}^{2}$

Ln(Share ownership +1$)_{t}$

Ln $(C E O \text { compensation }+1)_{t}$

Meeting frequency $(I d)_{t}$

Meeting frequency (Firm) ${ }_{t}$

Duality $_{t}$

Board sizet

Board composition(Ind\%) ${ }_{t}$

Cash holdingst

Ln(Total Assets) $)_{t}$

\section{Description}

The dummy variable equals to 1 if it is the first time this individual serves as a board of director in a firm in the year $t$ and 0 otherwise.

The dummy variable equals to 1 if if a board of director had or has an administrative ranking in Chinese political system in the year $t$ and 0 otherwise.

The dummy variable equals to 1 if a board of director is CEO or COB in the year $t$ and 0 otherwise. The age of a board of director in the year $t$.

The square of age of a board of director in the year $t$.

The logarithm of a board of director's share holding plus 1 in the year $t$.

The logarithm of CEO compensation plus 1 in the year $t$.

The number of board meetings for an independent director in the year $t$.

The number of board meetings for a firm in the year $t$.

The dummy variable equals to 1 if the CEO and chairman is the same person in the year $t$ and 0 otherwise.

The number of directors on board in the year $t$.

The ratio of independent directors on board in the year $t$.

The cash and marketable security divided by the book value of total assets in the year $t$.

The logarithm of book values of assets in the year $t$. 
Table 1.9: Variable definitions

Variable

Description

Book leverage $_{t}$

The ratio of book value of debts to book value of assets in the year $t$.

$R O A_{t-1}$

The net income divided by the book value of total assets in the year $t-1$.

Stock volatility t-1

The variance of monthly stock returns in the year $t-1$. 
Chapter 2

Rookie directors and firm

performance: Evidence from China 


\section{$2.1 \quad$ Introduction}

Rookie directors are an important supply of talent to corporate boards. According to Kang, Kim, and Low (2016), rookie directors account for almost one-third of new directors who join corporate boards in the U.S.. Rookie directors are even more important in China where the director tenure is restricted. ${ }^{1}$ From 2008 to 2014 in China, more than 26.8\% of independent directors and $60 \%$ of newly appointed independent directors are rookie independent directors. Despite the common use of rookie directors on corporate boards, there is limited research on their influence on corporate governance and firm performance. The only notable study is Kang, Kim, and Low (2016) who find that in the U.S. rookie independent directors positively influence board functions and firm values. However, the aforementioned findings do not provide clear guidance in the case of China, where the ownership structure and governance issues differ from those in the U.S. (Jiang and Kim, 2015). This study examines the influence of rookie independent directors on board functions and firm performance in China.

The value of rookie independent directors may be compromised by their limited board experience, which may hinder their coordination with management and ability to think strategically, restricting their ability to provide management guidance on the operation of the firm (Kang, Kim, and Low, 2016). Consistent with this view, Ahern and Dittmar (2012) find that a female director quota decreases firm values in Norway, which is consistent with a positive relation between experience and ability. ${ }^{2}$ Rookie independent directors have no track record in the director labor market. Their performance as rookie directors builds their reputation for additional appointments as independent directors. Thus, the career concern model suggests that rookie independent directors are more motivated than seasoned independent directors to develop reputations as diligent directors (Holmstrom, 1982).

Given the benefits and costs associated with rookie independent directors, their net effect on board functioning and firm performance is an empirical question. Our study addresses the following questions. Are rookie independent directors more diligent directors? What is the overall impact of rookie independent directors on firm operating performance? What are the potential channels through which rookie independent directors affect firm performance? Are rookie independent directors rewarded more for their efforts? What kind of firms benefit more from rookie independent directors?

We first explore whether rookie independent directors are more diligent directors. Because board meeting attendance is considered one of the major responsibilities of independent directors, we investigate the board meeting attendance of rookie independent directors.

\footnotetext{
${ }^{1}$ In China, an independent director is allowed to serve a maximum of six years in a firm. Sees Jiang, Wan, and Zhao (2015) for more detailed discussions on director tenure restriction in China.

${ }^{2}$ Espen, Knut, and Karin (2018) find the results of Ahern and Dittmar (2012) statistical insignificance in a replication study.
} 
In the prior literature, board meeting attendance is used as a primary measure of director commitment and monitoring effectiveness (Masulis, Wang, and Xie, 2012, Cai, Garner, and Walkling, 2009). Following Kang, Kim, and Low (2016), we define rookie independent directors as independent directors who have at least three years boardroom experience. We compare the board meeting attendance records of rookie independent directors to seasoned independent directors. We find that rookie independent directors are more likely than seasoned independent directors to attend board meetings. This evidence is consistent with the idea that career ambition motivates rookie independent directors to work harder than seasoned independent directors. In economic terms, the probability of rookie independent directors missing any board meeting is $1.2 \%$ lower than that of seasoned independent directors, which is equivalent to a $6.6 \%$ decrease from the average probability of board meeting absences of $18.3 \%$. Likewise, the board meeting absence rate of rookie independent directors is $0.5 \%$ lower than that of seasoned independent directors, which is equivalent to a $14.7 \%$ decrease from the average board meeting absence rate of $3.4 \%$.

We then examine the influence of rookie independent directors on firm operating performance. We find that firms with rookie independent directors outperform their counterparts as measured by both return on sales (ROS) and return on assets (ROA). In economic terms, firms with a majority of rookie independent directors outperform firms with a minority of rookie independent directors by ROS of $1.6 \%$ and ROA of $0.5 \%$, which represents a $26.2 \%$ increase from the mean ROS of $6.1 \%$ and a $13.5 \%$ increase from the mean ROA of $3.7 \%$. In the robustness section, we re-estimate the regression of rookie directors on firm performance using an alternative measure of $R O S_{t}$ and $R O A_{t}$. We replace the net income by EBITDA to calculate $R O S_{t}$ and $R O A_{t}$. Our results are robust to this alternative measure of $R O S_{t}$ and $R O A_{t}{ }^{3}$

Next, we investigate a potential channel through which rookie independent directors improve firm operating performance. An important role of independent directors is to monitor management. Therefore, if they are more effective monitors, rookie independent directors may improve the firm operating performance. Ownership structure affects independent director responsibilities. For example, in the U.S. and UK, ownership of listed firms is dispersed and the main agency conflict is between the managers and shareholders. Correspondingly, an important objective of independent directors is to hold managers accountable for performance. Consistent with this view, Kang, Kim, and Low (2016) find that in the U.S. rookie independent directors increase the pay-performance sensitivity of CEOs. In contrast, in China, the ownership of listed firms is highly concentrated and the main agency conflict is between the minority and controlling shareholders. Thus, the main governance issue in China is wealth expropriation of controlling shareholders from the firm's minority shareholders. Therefore, the main monitoring function of independent directors in

\footnotetext{
${ }^{3}$ In robustness testing, we find that our results hold using regressions with instrument variable.
} 
China is to protect minority shareholders from the wealth expropriation of the controlling shareholders, a phenomenon commonly referred as "tunneling" or "self-dealing" (Jiang, Lee, and Yue, 2010). ${ }^{4}$ We test the relation between the presence of rookie independent directors and tunneling to controlling shareholders. We find that the presence of rookie independent directors reduces tunneling to controlling shareholders. This evidence suggests that rookie independent directors are more effective monitors than seasoned independent directors. In economic terms, firms with a majority of rookie independent directors (relative to firms with a minority of rookie independent directors) decrease tunneling by $0.3 \%$ from total assets, a decrease is equivalent to 23.1 million CNY in dollar terms (3.4 million USD with the exchange rate of $6.8 \mathrm{CNY} / \mathrm{USD}) .^{5}$

We then examine the potential benefits to rookie independent directors from their efforts. Yermack (2005) suggests that retaining the current directorship is one of the major motivations for the board of directors to work hard. We find that board meeting absence reduces the likelihood of independent directors retaining their current directorships next year. Also, this negative effect of board meeting absence on the retention of current directorships is stronger for rookie independent directors than seasoned independent directors.

We investigate the characteristics of firms that benefit most from rookie independent directors. Prior literature finds that the tunneling to controlling shareholders is more common in non-state-owned firms than in state-owned firms (Jiang, Lee, and Yue, 2010). If rookie independent directors lessen tunneling to controlling shareholders, firms more vulnerable to tunneling (non-state-owned firms) should benefit more from the rookie independent directors. Consistent with this idea, we find that in China the rookie independent directors improve firm performance more in non-state-owned firms than in state-owned firms.

Prior literature suggests the importance of busy boards on corporate governance and firm performance (Core, Holthausen, and Larcker, 1999, Adams and Ferreira, 2008, Fich and Shivdasani, 2006, Ferris, Jagannathan, and Pritchard, 2003, Field, Lowry, and Mkrtchyan, 2013). The relatively low board experience of rookie directors makes them likely to be non-busy directors. This negative correlation between rookie directors and busy directors raises a concern that the positive influence of rookie directors on firm performance is driven by the presence of fewer busy independent directors rather than more rookie independent directors on board. To assess if our findings are driven by board busyness, we control for the busyness of the board in all firm level regressions. Consistent with Giannetti, Liao, and $\mathrm{Yu}$ (2015) and Liang, Xu, and Jiraporn (2013), we find that in China board busyness does not affect firm performance.

\footnotetext{
${ }^{4}$ In 2001, when the independent director system was introduced to Chinese listed firms by CSRC, they explicitly state that the primary and legally explicit responsibility of independent directors is to monitor large controlling shareholders on behalf of minority shareholders. Sees Guidelines for Introducing Independent Directors to the Board of Directors of Listed Companies 2001.

${ }^{5} \mathrm{~A} 0.3 \%$ decrease of $\mathrm{ORECTA}(\%)_{t}$ from a firm with average total assets of 7.674 billion CNY equals to 23.1 million CNY.
} 
OLS regressions of firm performance are subject to simultaneity issues. We include several econometric methods to address endogeneity issues. The first method is firm fixed effects, which control for any time-invariant firm-specific factors related to the presence of rookie independent directors and firm performance. Reverse causality between the presence of rookie independent directors and firm performance is another endogeneity concern. To test reverse causality, we investigate whether firm characteristics at year $t-1$ predict the rookie independent directors appointments at year $t$. We find that the firm performance at year $t-1$ does not predict the rookie independent directors appointments at year $t$, suggesting that our results are unlikely to be driven by reverse causality. To further address endogeneity, we estimate our firm performance regressions using instrumental variables. We construct two instrumental variables. The first instrument Retire director $(\%)_{t-1}$ is the mean value of the percent of independent directors of other firms headquartered in the same city leaving their boards due to the term limits at year $t-1$. The second instrument First-year director $(\%)_{t-1}$ is the mean value of the percent of first-year directors of other firms headquartered in the same city at year $t-1$. Both instruments explain the appointment of rookie directors (relevant condition), but are unrelated to firm performance (over-identification condition). Our results are qualitatively unchanged using instrumental variable two-stage estimation.

The remainder of the paper is organized as follows. Section 2 discusses relevant literature and develops the hypothesis. Section 3 provides sample and variables construction. Section 4 presents the empirical method for testing and reports the main empirical results. Section 5 presents the robustness tests. The final section concludes the paper.

\section{$2.2 \quad$ Literature and hypothesis development}

Rookie independent directors have no track record in the independent directorial labor market. Their performance as rookie directors likely influences their future careers as independent directors. Thus, career concerns suggest that rookie independent directors have stronger incentives than seasoned independent directors to develop reputations as diligent directors (Holmstrom, 1982, Yermack, 2005). Board meetings are the main channel for independent directors to collect information, monitor the management and make decisions (Adams and Ferreira, 2008, Masulis, Wang, and Xie, 2012, Chou, Chung, and Yin, 2013, Masulis and Mobbs, 2014). Prior literature considers board meeting attendance a primary measure of directors' commitment to their directorship responsibilities (Masulis, Wang, and Xie, 2012, Cai, Garner, and Walkling, 2009, Masulis and Mobbs, 2014). If rookie independent directors are more motivated than seasoned independent directors to work as diligent directors, rookie independent directors are more likely to attend board meetings than seasoned independent directors. This leads us to the director-level hypothesis: 
H1: Rookie independent directors are less likely to miss board meetings than seasoned independent directors, ceteris paribus.

In contrast, rookie independent directors have less board experience. This more limited board experience may restrict their ability to coordinate with management and think strategically, which compromises their ability to provide management guidance on the operation of the firm (Kang, Kim, and Low, 2016). Consistent with a positive relation between experience and ability, Ahern and Dittmar (2012) find that a female director quota decreases firm values in Norway. Given the possible benefits and costs associated with rookie independent directors, their net effect on overall board functioning and firm performance is an empirical question. Consistent with the reputation story, Kang, Kim, and Low (2016) find that, in the U.S., rookie independent directors positively impact corporate governance and firm value. Their evidence leads us to conjecture that:

H2: Firms with more rookie independent directors have better performance than their counterparts, ceteris paribus.

In China, one of the main agency conflicts is between the minority and controlling shareholders. An important governance issue is the wealth expropriation of controlling shareholders from the minority shareholders. Thus, minimizing tunneling to the controlling shareholders is considered one of the main responsibilities of independent directors. ${ }^{6}$ Consistent with this view, prior literature on China considers minimizing tunneling to controlling shareholders as one of the primary measures of a director's commitment and monitoring efficiency (Liu, Miletkov, Wei, and Yang, 2015, He and Luo, 2018). If rookie independent directors have more incentive than seasoned independent directors to work as diligent monitors, there should be a negative relation between the presence of rookie independent directors and firm tunneling. Therefore, our hypothesis is:

H3: Rookie independent directors decrease tunneling to the controlling shareholders, ceteris paribus.

Prior literature suggests that independent directors are rewarded for their efforts by the internal markets (the firms) and the external directorial labor markets. For example, Yermack (2005) finds that after a firm experiences high stock returns its independent directors are less likely to lose their current directorships and more likely to obtain additional directorships from other firms. Similarly, Jiang, Wan, and Zhao (2015) find that in China independent directors, who are diligent monitors, receive additional directorships from other firms in the future. ${ }^{7}$ Consistent with these studies, our hypothesis is:

\footnotetext{
${ }^{6}$ In 2001, when the independent director system was introduced to Chinese listed firms by CSRC, they explicitly state that the primary and legally explicit responsibility of independent directors is to monitor large controlling shareholders on behalf of minority shareholders. See Guidelines for Introducing Independent Directors to the Board of Directors of Listed Companies 2001.

${ }^{7}$ Jiang, Wan, and Zhao (2015) measure the monitoring of independent directors by their voting dissent in board meetings.
} 
H4a: Directors who attend more board meetings are more likely to retain their current directorships, ceteris paribus.

Furthermore, Kang, Kim, and Low (2016) find the positive relationship between firm performance and the likelihood of obtaining additional directorships is stronger for rookie independent directors than seasoned independent directors. This evidence suggests that the marginal benefit of effort is higher for rookie independent directors than seasoned independent directors. This leads us to the hypothesis:

H4b: The negative relationship between board meeting attendance and director turnover is stronger for rookie independent directors than seasoned independent directors, ceteris paribus.

\subsection{Sample and variable construction}

\subsubsection{Sample}

We collect the independent director profile, meeting attendance record, turnover record, board composition and financial data from the Chinese Listed Firms Research Series database (CSMAR). ${ }^{8}$ We define independent directors basing on the classification from the CSMAR database, which is a legal definition of director independence. ${ }^{9}$ The sample for this study consists of all firms listed on the Shanghai Stock Exchange (SSE) and Shenzhen Stock Exchange (SZSE) for the period 2008-2014. We start the sample from 2008 since the political background data is available in CSMAR after 2008.

Following the prior literature, we exclude firms from financial and public utility industries in our sample. We also exclude firm-year observations with negative equity or negative sales. To avoid outliers, we winsorize all firm financial characteristics at the top and bottom $0.5 \%$ percentiles. Table 2.12 shows all variable definitions. Our final sample consists of 42,608 director-firm years and 12,433 firm-year observations. The number of firms in our sample ranges from 1,238 in 2008 to 2,189 in 2014. In the following sections, we construct all variables. ${ }^{10}$

\footnotetext{
${ }^{8}$ The CSMAR database is widely regarded as the most comprehensive and authoritative database to study corporate finance and corporate governance in Chinese listed firms. According to a report issued by ShenZhen GTA, the CSMAR database has been used in papers published in a dozen leading international journals including Journal of Finance, Journal of Financial Economics, Journal of Financial and Quantitative Analysis and Review of Financial Studies.

${ }^{9}$ See Section 1.2.3 for a detail discussion on the legal definition of director independence.

${ }^{10}$ Please see Table 2.12 for a summary of all variable definitions.
} 


\subsubsection{Dependent variables}

\section{Board meeting attendance}

In China, publicly listed firms are required to disclose the board meeting attendance of their independent directors in their annual reports. The meeting attendance record in China discloses: 1) the number of board meetings that an independent director is required to attend during a year; 2) the number of board meetings that an independent director attended during a year (both physical and teleconference attendance); 3) the number of board meetings that an independent director misses or authorizes a representative to attend during a year. Compared to the US, board meeting attendance data in China is more precise and comprehensive. ${ }^{11}$ We classify both "misses a board meeting" and "authorizes a representative to attend a board meeting" as a board meeting absence because in both scenarios the director avoids the effort to attend the board meeting. Also, the previous literature finds that both "misses a board meeting" and "authorizes a representative to attend a board meeting" have similar outcomes on firm operations. For example, Chou, Chung, and Yin (2013) find that in Taiwan, the board of directors improve firm performance through the board meetings they attend, while this positive effect disappears when the directors miss or send a representative to attend the board meetings.

We use the variables Meeting absence $(0 / 1)_{t}$ and Meeting absence $(\%)_{t}$ as our measures of independent director board meeting absence. Meeting absence $(0 / 1)_{t}$ is a dummy variable that equals to 1 if an independent director misses any board meeting during a year and 0 otherwise. Meeting absence $(\%)_{t}$ is the ratio of the number of board meetings missed scaled by the number of board meetings required during a year. Panel $A$ of Table 2.1 shows that $18.3 \%$ of independent directors miss at least one board meeting with an average board meeting absence rate 3.4\%. Compared to those in Chou, Chung, and Yin (2013), independent directors in Chinese listed firms exhibit relatively high board meeting attendance. ${ }^{12}$

\section{Firm performance}

Liu, Wei, and Xie (2014) suggest that neither return on equity (ROE) nor Tobin's Q are proper performance measures for Chinese listed firm. ${ }^{13}$ Following Liu, Wei, and Xie (2014),

\footnotetext{
${ }^{11}$ The US data is limited since it only discloses whether a director attends more than $75 \%$ of board meetings or not.

${ }^{12} \mathrm{Chou}$, Chung, and Yin (2013) find that independent directors in Taiwan listed firms only attend $70.8 \%$ of board meetings by themselves.

${ }^{13} \mathrm{Liu}, \mathrm{Wei}$, and Xie (2014) argue that, in China, return on equity (ROE) fails to correctly reflect firm financial performances since it is often manipulated to satisfy a seasonal equity offering requirement. In China, Tobin's Q is not considered a proper measure of firm financial performances since there are huge price gaps between tradable and non-tradable shares. Non-tradable shares are typical owned by the government and were acquired at prices substantially lower than the initial public offering prices. The non-tradable shares were not permitted to be traded in the secondary market before 2005. In 2005, listed firms were required to gradually convert their non-tradable shares into tradable shares due to the state ownership
} 
we measure firm accounting performance using return on sales (ROS) and return on assets (ROA). We calculate $R O S_{t}$ as net income divided by sales and $R O A_{t}$ as net income divided by total assets. Panel $C$ of Table 2.1 reports that the mean of $R O S_{t}$ and $R O A_{t}$ is $6.1 \%$ and $3.7 \%$ respectively.

\section{Tunneling to controlling shareholders}

Jiang, Lee, and Yue (2010) document that in China, the controlling shareholders often divert corporate resources from the listed firms to the controlling shareholders' other entities (most of which are unlisted) through inter-corporate loans. These inter-corporate loans are typically reported on the balance sheets of lending firms under the accounting item "Other receivables". ${ }^{14}$ In practice, controlling shareholders incur no interest charge on these inter-corporate loans, and even worse, the controlling shareholders often fail to repay the principal (Jiang, Lee, and Yue, 2010, He and Luo, 2018). Jiang, Lee, and Yue (2010) show that firms with high ORECTA $(\%)_{t}$ (other receivables scaled by total assets) are more likely to experience poorer operating performance and face financial distress in the future.

We follow Jiang, Lee, and Yue (2010) and construct ORECTA(\%) $t$ as our measure of tunneling to controlling shareholders, where $\operatorname{ORECTA}(\%)_{t}$ equals other receivables scaled by total assets. Panel $C$ of Table 2.1 shows the mean ORECTA(\%) ${ }_{t}$ of Chinese listed firms is $1.9 \%$ from 2008 to 2014, which is lower than that in Jiang, Lee, and Yue (2010) but similar to that in Qian and Yeung (2015). ${ }^{15}$ The decline of ORECTA(\%) $t$ is possibly due to the new regulation. Since 2006, the Chinese Security Regulatory Committee (CSRC) requires firms to disclose the actual amount of inter-corporate loans by the controlling shareholders. Despite the regulation, Qian and Yeung (2015) find the number of firms with non-zero ORECTA keeps increasing. This suggests that although the regulation appears to have decreased the magnitude of tunneling, the practice remains common and continues to spread.

\section{Director turnover}

Following Yermack (2005), our measure Turnover $(0 / 1)_{t+1}$ is a dummy variable equals to 1 for an observation in year $t+1$ if an independent director does not appear in the annual report in year $t+2$ and 0 otherwise. We exclude observations from delisted firms. Because of term limits, we excluded the directors leaving the board at year 6 or year 7. Panel A of Table 2.1 reports the average turnover rate of independent directors is $14.3 \%$.

reform. The ownership reform was basically completed by 2007. However, there are still restrictions for the former non-tradable shareholders on trading their shares. For example, the percentage of shares permitted to be traded and the lockup period

${ }^{14}$ Unlike "Accounts receivables", "Other receivables" does not record ordinary business transactions.

${ }^{15}$ Jiang, Lee, and Yue (2010) report that the average ORECTA(\%) $t$ was $8.1 \%$ from 1996 to 2004. Qian and Yeung (2015) report that the average ORECTA(\%) decreased to $2.18 \%$ in 2009 


\subsubsection{Variables of interest}

Following Kang, Kim, and Low (2016), we classify independent directors into rookie independent directors and seasoned independent directors based on their board experience. We follow Kang, Kim, and Low (2016) and do not distinguish between directorial experience as an independent director or as an inside director. We define rookie independent directors as independent directors who have less than three years of boardroom experience and seasoned independent directors as independent directors who have three years or more boardroom experience.

In directorship level analysis, we use Rookie director $(0 / 1)_{t}$ to measure rookie independent directors. Rookie director $(0 / 1)_{t}$ is a dummy variable that equals 1 if an independent director has less than three years of boardroom experience and 0 otherwise. Panel $A$ of Table 2.1 shows that $25.7 \%$ of independent directors are rookie independent directors. In firm level analysis, we use either Rookie director $(\%)_{t}$ or Rookie board $(0 / 1)_{t}$ to measure the rookie board. Rookie director $(\%)_{t}$ is the ratio of rookie independent directors that serve on the board. Rookie board $(0 / 1)_{t}$ is a dummy variable that equals 1 if the majority of independent directors are rookies and 0 otherwise. Panel B of Table 2.1 shows that for $18.3 \%$ of boards the majority of independent directors are rookies.

\subsubsection{Control variables}

In the directorship level regressions, which are used to study the board meeting attendance and director turnover, we control for the director, board and firm features. The directorship level control variables include Woman(0/1), Age(Ten years) ${ }_{t}$, Tenure in firm(Years) , Busy director $(0 / 1)_{t}$, Ln(Director compensation) $)_{t}$, Political backgrounds(0/1) $t$ and Meeting

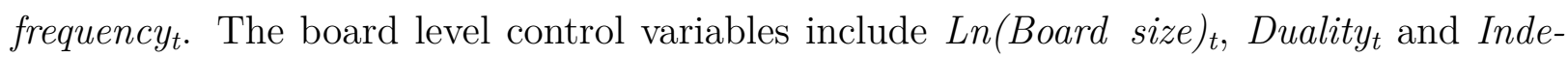
pendent director $(\%)_{t}$. The firm level control variables include State-owned $(0 / 1)_{t}$, Largest shareholder(\%), Ln(Sales) $)_{t}$, Book leverage $t_{t}$ and $R O A_{t}$.

In the firm level regressions, which are used to study firm performance and tunneling, we control for a wide array of firm characteristics including board composition, ownership structure and financial characteristics that prior literature has shown to be related to firm performance or tunneling. ${ }^{16}$ The board level control variables include Women director(\%), Busy director $(\%)_{t}$, Ln(Board size $)_{t}$, Duality $y_{t}$ and Independent director(\%) $)_{t}$. The firm level control variables include State-owned $(0 / 1)_{t}$, Largest shareholder $(\%)_{t}$, Ln (Sales $)_{t}$, Ln(Firmage) $)_{t}$, Book leverage $_{t}, R O A_{t-1}$ and $R \& D(\%)_{t}$.

\footnotetext{
${ }^{16}$ In the regression on tunneling, we control for board composition and a similar set of control variables as in Jiang, Lee, and Yue (2010).
} 
In Table 2.1, we report the summary statistics of control variables. In China, $14.6 \%$ of independent directors are female and $26 \%$ are busy directors. ${ }^{17}$ In our sample, the average independent director is 53.03 years old, serves on the current firm for 3.26 years and has 1.96 directorships. The average annual independent director compensation is 55,383 CNY (equivalent to 8,145 USD with the exchange rate of $6.8 \mathrm{CNY} / \mathrm{USD}$ ), which is similar to that in Chen and Keefe (2018). ${ }^{18}$ In our sample, $39.8 \%$ of independent directors have political backgrounds. In China, the average board has eight board meetings each years.

On average, the Chinese boards have 8.8 members. In $23.8 \%$ of their boards, the CEO and chairman are the same person. $37 \%$ of board members are independent directors. In our sample, $43.6 \%$ of firms are state-owned. The largest shareholders on average own $36.1 \%$ of the shares of the listed firm. Chinese listed firms are relatively young, with an average firm age equals to 15 years. The average firm has total book assets of 7.67 billion CNY (equivalent to 1.13 billion USD with the exchange rate of $6.8 \mathrm{CNY/USD),} \mathrm{sales} \mathrm{of} 4.99$ billion CNY (equivalent to 0.73 billion USD with the exchange rate of $6.8 \mathrm{CNY} / \mathrm{USD}$ ), book leverage of $46.6 \%$ and research and development expenditure scaled by sales of $0.3 \%$. Table 2.2 provides the correlation coefficients between key variables in the whole sample.

In Table 2.2, we report the correlation matrix of all variables from the regression on firm performance. Neither Rookie director $(\%)_{t}$ nor Rookie board $(0 / 1)_{t}$ is highly correlated with any of the control variables. Specifically, the correlation coefficient between Rookie director $(\%)_{t}$ and Busy director $(\%)_{t}$ is $-12.4 \%$, indicating that the measure of rookie director and busy director is not a mechanical relationship.

\subsection{Test approach and results}

\subsubsection{Are rookie independent directors more diligent directors?}

In this section, we explore whether rookie independent directors are more diligent than seasoned independent directors. Following the prior literature, we measure diligence by board meeting attendance (Adams and Ferreira, 2008, Masulis, Wang, and Xie, 2012, Chou, Chung, and Yin, 2013, Cai, Garner, and Walkling, 2009).

In our sample, about $15.9 \%$ of rookie independent directors and $19.3 \%$ of seasoned independent directors miss at least one board meeting. This difference of $3.4 \%$ is statistically significant at less than the $1 \%$ level. Similarly, the average board meeting absence rate for rookie independent directors is $3.0 \%$, while the average board meeting absence rate for seasoned independent directors is $3.6 \%$. That difference of $0.6 \%$ is statistically significant

\footnotetext{
${ }^{17}$ Giannetti, Liao, and $\mathrm{Yu}(2015)$ find that in China $16.2 \%$ of independent directors are busy directors from 1999 to 2009.

${ }^{18}$ Chen and Keefe (2018) find that, during 2005-2015, the average annual compensation for independent directors equals to $57,654 \mathrm{CNY}$ (equivalent to 8,478 USD with the exchange rate of $6.8 \mathrm{CNY} / \mathrm{USD}$ ).
} 
at less than the $1 \%$ level. These sample statistics are consistent with Hypothesis 1, which advances that rookie independent directors are less likely to miss board meetings than seasoned independent directors.

To control for possible confounding factors, we estimate several linear probability models to better understand the board meeting attendance of rookie independent directors. The unit of observation is a director-firm-year. The regressions control for year, industry, firm, director and firm*year fixed effects. Our estimation equation is as follows:

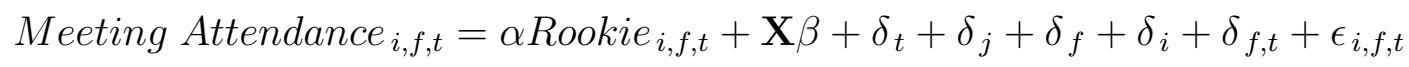

where $t$ represents the year, $j$ the industry, $f$ the firm and $i$ the director. The dependent variable is either Meeting absence $(0 / 1)_{t}$ or Meeting absence(\%). The variable of interest is Rookie director $(0 / 1)_{t}$. $\mathbf{X}$ is a matrix of control variables previously described in Section 2.3.4. $\delta_{t}, \delta_{j}, \delta_{f}, \delta_{i}$ and $\delta_{f t}$ denote year, industry, firm, director and firm*year fixed effects respectively. $\epsilon_{i j t}$ is the error term. To control for potential serial correlation, we use robust standard errors adjusted for firm-level clustering and heteroskedasticity. We apply linear probability regressions rather than logit regressions to study Meeting absence $(0 / 1)_{t}$ for two reasons. ${ }^{19}$ First, the logit regressions with fixed effects (conditional logit regressions) require within group variation for the dependent variables. For example, the logit regressions with firm fixed effects require the dependent variables to be different in a firm. ${ }^{20}$ Therefore, the estimation of logit regressions with fixed effects reduces sample size. Second, the marginal effects from logit regressions can not be properly estimated when fixed effects are included and the odd ratio is difficult to interpret. ${ }^{21}$ We exclude observations in which an independent director has served on a board for less than a year. ${ }^{22}$

Table 2.3 reports regression results investigating the board meeting attendance of rookie independent directors. In Columns (1) to (4), the dependent variable is Meeting absence $(0 / 1)_{t}$. In Columns (5) to (8), the dependent variable is Meeting absence(\%). In Columns (1) and (5), we include year and industry fixed effects in our regressions. In Columns (2) and (6), we include year and firm fixed effects in our regressions. The firm fixed effects control for any time-invariant firm-specific factors that affect the board meeting attendance of independent directors. In Columns (3) and (7), we include year, industry and director fixed effects into our regressions. The director fixed effects control for any time-invariant director-specific

\footnotetext{
${ }^{19}$ In unreported tables, we estimate Meeting absence $(0 / 1)_{t}$ using logit regressions with year, industry, firm, director and firm*year fixed effects. The results from logit regressions are quantitatively similar to those from linear probability regressions. Therefore, our results are robust to the model selection.

${ }^{20}$ In the estimation, observations not fitting the requirement are deleted.

${ }^{21}$ Simonetta and Alita (2015) suggest that, for logit regressions with fixed effects, marginal effects can only be estimated for the special case where the unobserved heterogeneity is zero (fixed effects equal to zero). However, these marginal effects are of little value.

${ }^{22}$ After excluding those observations, board meeting attendance data in the remaining sample would have the same duration (one year duration for all remaining observations).
} 
factors that affect board meeting attendance of independent directors. In Columns (4) and (8), we include the firm*year fixed effects into our regressions. The regressions with firm*year fixed effects only allow variation of board meeting attendance among directors serving on the same board at the same time. Therefore, any found difference in board meeting attendance is due to the variation of director characteristics. For example, whether an independent director is a rookie or not.

In Column (1), the coefficient associated with Rookie director $(0 / 1)_{t}$ is negative and statistically significant at the less than $5 \%$ level in explaining the probability of board meeting absences Meeting absence(0/1)t. The above results support Hypothesis 1 that rookie independent directors are more diligent directors. The marginal effect of the coefficient associated with Rookie director $(0 / 1)_{t}$ is -0.012 , suggesting that rookie independent directors are $1.2 \%$ less likely to miss at least one board meeting than seasoned independent directors. A $1.2 \%$ decrease represents a $6.6 \%$ relative decrease from the average probability of board meeting absences of $18.3 \%$.

In Column (5), the coefficient associated with Rookie director $(0 / 1)_{t}$ is negative and statistically significant at the less than $1 \%$ level in explaining the board meeting absence rate Meeting absence $(\%)_{t}$. The above result suggests that rookie independent directors have a lower board meeting absence rate than seasoned independent directors. In Column (5), the coefficient associated with Rookie director $(0 / 1)_{t}$ is -0.005 , suggesting that the board meeting absence rate of rookie independent directors is $0.5 \%$ lower than that of seasoned independent directors. A $0.5 \%$ decrease represents $14.7 \%$ relative decrease from the average board meeting absence rate of $3.4 \%$.

In Columns (2) to (4) and Columns (6) to (8), the coefficients associated with Rookie director $(0 / 1)_{t}$ are negative and statistically significant at the less than $5 \%$ level in explaining Meeting absence $(0 / 1)_{t}$ and Meeting absence $(\%)_{t}$. Therefore, our results are robust to year, industry, firm, director and firm*year fixed effects.

\subsubsection{Rookie directors and firm performance}

In this section, we examine the relationship between rookie independent directors and firm performance. We estimate regressions of firm performance against the presence of rookie independent directors. The unit of observation for the regression is a firm-year. We include the firm fixed effects to control for any time-invariant firm factors that relate to both firm performance and the presence of rookie independent directors. Our estimation equation is as follows:

$$
\text { Firm Performance }_{f, t}=\alpha \text { Rookie }_{f, t}+\mathbf{X} \beta+\delta_{t}+\delta_{f}+\epsilon_{f, t}
$$

where $t$ represents the year and $f$ the firm. In regressions, the dependent variable is either $R O S_{t}$ (return on sales) or $R O A_{t}$ (return on assets). The variable of interest is either Rookie 
director $(\%)_{t}$ or Rookie board(0/1) $)_{t} \mathbf{X}$ is a matrix of control variables previously described in Section 2.3.4. $\delta_{t}$ and $\delta_{f}$ denote year and firm fixed effects respectively. $\epsilon_{f t}$ is the error term. To control for potential serial correlation, we use robust standard errors adjusted for firm-level clustering and heteroskedasticity.

Table 2.4 presents estimation results of equation (2.2). In Columns (1) and (2), the dependent variable is $R O S_{t}$. In Columns (3) and (4), the dependent variable is $R O A_{t}$. In Columns (1) and (3), we find that the coefficients associated with Rookie director $(\%)_{t}$ are positive and statistically significant at the less than $5 \%$ level in explaining $R O S_{t}$ and $R O A_{t}$. In Columns (2) and (4), we find that the coefficients associated with Rookie board $(0 / 1)_{t}$ are positive and statistically significant at the less than $1 \%$ level in explaining $R O S_{t}$ and $R O A_{t}$. These results are consistent with Hypothesis 2, which advances that firms with more rookie independent directors perform better.

Columns (2) and (4) interpret the economic importance of our results. Column (2) shows that the $R O S_{t}$ of firms with a majority of rookie independent directors is $1.6 \%$ higher than the firms without a majority of rookie independent directors, which is equivalent to an increase of $26.2 \%$ relative to the average $R O S_{t} 6.1 \%$. Column (4) shows that the $R O A_{t}$ of firms with a majority of rookie independent directors is about $0.5 \%$ higher than their counterparts, which is equivalent to an increase of $13.5 \%$ relative to the average $R O A_{t}$ of $3.7 \%$.

\subsubsection{What are the potential channels through which rookie in- dependent directors affect firm performance?}

In this section, we study the potential channels through which rookie independent directors may improve firm performance. In China, the main agency conflict is between minority and controlling shareholders and the corresponding monitoring focus of independent directors is to decrease tunneling to controlling shareholders. Thus, rookie independent directors may improve firm performance by decreasing tunneling through more efficient monitoring.

We estimate the presence of rookie independent directors on the tunneling behavior of controlling shareholders. The unit of observation for the regressions is a firm-year. We include the year, industry and firm fixed effects. Our estimation equation is as follows:

$$
\operatorname{ORECTA}_{f, t}=\alpha \text { Rookie }_{f, t}+\mathbf{X} \beta+\delta_{t}+\delta_{j}+\delta_{f}+\epsilon_{f, t}
$$

where $t$ represents the year, $j$ the industry and $f$ the firm. In the regressions, the dependent variable is $\operatorname{ORECTA}(\%)_{t}$. The variable of interest is either Rookie director(\%) $t$ or Rookie $\operatorname{board}(0 / 1)_{t} . \mathrm{X}$ is a matrix of control variables previously described in Section 2.3.4. $\delta_{t}, \delta_{j}$ and $\delta_{f}$ denote year, industry and firm fixed effects respectively. $\epsilon_{f t}$ is the error term. To 
control for potential serial correlation, we use robust standard errors adjusted for firm-level clustering and heteroskedasticity.

Table 2.5 presents the results from regressions investigating the association between rookie independent directors and tunneling to controlling shareholders. In Columns (1) and (2), we include year and industry fixed effects. In Columns (3) and (4), we include year and firm fixed effects. The firm fixed effects control for any time-invariant firm factors that relate to both the presence of rookie independent directors and tunneling to controlling shareholders.

In Column (1), the coefficient associated with Rookie director(\%) $)_{t}$ is negative and statistically significant at the less than $5 \%$ level in explaining ORECTA(\%)t. Similarly, in Column (2), the coefficient associated with Rookie board $(0 / 1)_{t}$ is negative and statistically significant at the less than $5 \%$ level in explaining ORECTA $(\%)_{t}$. These results are consistent with Hypothesis 3 that rookie independent directors decrease tunneling to controlling shareholders. Column (2) shows that, in economic terms, the ORECTA(\%) t $_{\text {of firms with }}$ a majority of rookie independent directors is about $0.3 \%$ lower than that of the firms without a majority of rookie independent directors, a decrease of tunneling equaling to $15.8 \%$ relative decrease from the average $\operatorname{ORECTA}(\%)_{t}$ of $1.9 \%$. In dollar terms, this decrease of tunneling equals to 23.1 million CNY (equivalent to 3.4 million USD with the exchange rate of $6.8 \mathrm{CNY} / \mathrm{USD})$.

In Columns (3) and (4), when firm fixed effects are controlled, the coefficient associated with Rookie director $(\%)_{t}$ is negative and statistically significant at the less than $5 \%$ level and the coefficient associated with Rookie board $(0 / 1)_{t}$ is negative and statistically significant at the less than $1 \%$ level in explaining ORECTA(\%)t. Our results are robust to the inclusion of firm fixed effects.

\subsubsection{Are rookie independent directors rewarded more for their efforts?}

In this section, we examine the potential benefits to rookie independent directors from attending meetings. We estimate linear probability models regarding the effects of meeting attendance on director turnover. ${ }^{23}$ The unit of observation for the regression is a director-

\footnotetext{
${ }^{23}$ See discussion in Section 2.4.1 on the reasons for linear probability models. In unreported tables, we estimate Turnover $(0 / 1)_{t+1}$ using logit regressions with year, industry, firm, director and firm*year fixed effects. The results from logit regressions are quantitatively similar to those from linear probability regressions.
} 
firm-year. The regressions control for year, industry, firm, director and firm*year fixed effects. Our estimation equation is as follows:

$$
\begin{aligned}
& \text { Turnover }_{i, f, t+1}=\alpha_{\mathbf{1}} \text { Rookie }_{i, f, t}+\alpha_{\mathbf{2}} \text { Meeting Attendance }_{i, f, t} \\
& +\alpha_{\mathbf{3}} \text { Rookie }_{i, f, t} * \text { Meeting Attendance }_{i, f, t}+\mathbf{X} \beta+\delta_{t}+\delta_{j}+\delta_{f}+\delta_{i}+\delta_{f, t}+\epsilon_{i, f, t}
\end{aligned}
$$

where $t$ represents the year, $j$ the industry, $f$ the firm and $i$ the director. The dependent variable is Turnover $(0 / 1)_{t+1}$. The variables of interest are Rookie director $(0 / 1)_{t}$, Meeting absence $(0 / 1)_{t}$ and its interaction term. The interaction term between Rookie director $(0 / 1)_{t}$ and Meeting absence $(0 / 1)_{t}$ isolates the sensitivity of rookie independent directors (vs seasoned independent directors) of board meeting attendance on director turnover. A positive (negative) interaction term between Rookie director $(0 / 1)_{t}$ and Meeting absence $(0 / 1)_{t}$ indicates that, if they miss a board meeting in year $t$, rookie independent directors are more (less) likely than seasoned independent directors to lose their directorship in year $t+1$. X is a matrix of control variables previously described in Section 2.3.4. $\delta_{t}, \delta_{j}, \delta_{f}, \delta_{i}$ and $\delta_{f t}$ denote year, industry, firm, director and firm*year fixed effects respectively. $\epsilon_{i f t}$ is the error term. To control for potential serial correlation, we use robust standard errors adjusted for firm-level clustering and heteroskedasticity.

Table 2.6 reports the estimation results of equation (2.4). In Column (2), we include year and firm fixed effects in our regressions. In Column (3), we include year, industry and director fixed effects into our regressions. In Column (4), we include the firm*year fixed effects into our regressions. ${ }^{24}$ In Column (1), the coefficient associated with Meeting absence $(0 / 1)_{t}$ is positive and statistically significant at the less than $1 \%$ level in explaining Turnover $(0 / 1)_{t+1}$, suggesting that missing board meetings reduces the likelihood of an independent director retaining a current directorship. This result is consistent with Hypothesis 4a that directors are more likely to secure their current directorships if they attend more board meetings. In Column (1), the interaction term between Rookie director $(0 / 1)_{t}$ and Meeting absence $(0 / 1)_{t}$ is positive and statistically significant at the $1 \%$ level in explaining Turnover $(0 / 1)_{t+1}$. This result is consistent with Hypothesis $4 \mathrm{~b}$ that the negative relationship between board meeting attendance and director turnover is stronger for rookie independent directors. The above evidence emphasizes the importance of rookie independent directors attending board meetings.

In Columns (2) and (4), when firm fixed effects are controlled, the coefficients associated with Meeting absence $(0 / 1)_{t}$ and interaction terms between Rookie director $(0 / 1)_{t}$ and Meeting absence $(0 / 1)_{t}$ are positive and statistically significant at the less than $1 \%$ level in explaining Turnover $(0 / 1)_{t+1}$. Therefore, our results are robust to the inclusion of firm fixed effects.

\footnotetext{
${ }^{24}$ See discussion in Section 2.4.1 on fixed effects.
} 
In Column (3), which controls for director fixed effects, the coefficient associated with Meeting absence $(0 / 1)_{t}$ is positive and statistically significant at the less than $1 \%$ level in explaining Turnover $(0 / 1)_{t+1}$ and the interaction term between Rookie director $(0 / 1)_{t}$ and Meeting absence $(0 / 1)_{t}$ is positive and statistically significant at the less than $10 \%$ level in explaining Turnover $(0 / 1)_{t+1}$. Thus, our results are robust with director variation.

If the director turnover is voluntary, a reverse causality problem may arise between board meeting attendance and director turnover. An independent director who plans to voluntarily step down from the board is less motivated, and may therefore stop attending regular board meetings. Due to data limitations, we can not distinguish forced turnover from voluntary turnover. ${ }^{25}$ However, in the director turnover regressions (equation (2.4)), we control for some variables relating to voluntary departure. For example, we include director age to control for the possibility that directors may voluntarily leave their boards for retirement. We include board meeting frequency and director busyness to control for the possibility that directors may voluntarily leave due to the workload. We include ROA to control for the possibility that directors may voluntarily leave due to the firm's poor performance. Thus, our results are robust with voluntary departure due to the above reasons.

\subsubsection{What kind of firms benefit more from rookie independent directors?}

In this section, we examine the characteristics of firms that benefit most from rookie independent directors. We show that the presence of rookie independent directors lessens tunneling to controlling shareholders. Prior literature finds that tunneling to controlling shareholders is more common in the non-state-owned firms than state-owned firms (Jiang, Lee, and Yue, 2010). Therefore, we predict that in China rookie independent directors improve firm performance in the non-state-owned firms more than state-owned firms.

Table 2.7 reports the results from regressions investigating the influence of rookie independent directors on firm performance between state-owned and non-state-owned firms. In Columns (1) and (3), the coefficients associated with Rookie director(\%) $t$ are positive and statistically significant at the less than $1 \%$ level in explaining $R O S_{t}$ and $R O A_{t}$. In Columns (2) and (4), the coefficients associated with Rookie board(0/1) $t$ are positive and statistically significant at the less than $1 \%$ level in explaining $R O S_{t}$ and $R O A_{t}$. These results suggest that non-state-owned firms with more rookie independent directors are associated with better performance.

In addition, in Columns (1) and (3), the interaction terms between State-owned $(0 / 1)_{t}$ and Rookie director $(\%)_{t}$ are negative and statistically significant at the less than $5 \%$ level

\footnotetext{
${ }^{25}$ The forced turnovers from delisted firms and term-limit regulation are observable. We delete these samples since these forced turnovers are mechanical.
} 
in explaining $R O S_{t}$ and $R O A_{t}$. In Columns (2) and (4), the interaction terms between State-owned $(0 / 1)_{t}$ and Rookie board $(0 / 1)_{t}$ are negative and statistically significant at the less than $5 \%$ level in explaining $R O S_{t}$ and $R O A_{t}$. These results indicate that the benefit of rookie independent directors on firm performance decreases in state-owned firms. The above evidence supports the prediction that in China rookie independent directors improve firm performance in the non-state-owned firms more than state-owned firms.

\subsection{Discussion and Robustness Tests}

\subsubsection{Rookie independent directors and director busyness}

Prior literature suggests that the influences of busy directors on firm performance is mixed. Consistent with a negative view, Core, Holthausen, and Larcker (1999) find that firms with busy directors are more likely to overpay their CEOs. Adams and Ferreira (2008) find that directors with multiple directorships are more likely to miss board meetings, suggesting that busy directors are less effective monitors. Fich and Shivdasani (2006) find that firms with a majority of busy directors exhibit lower market-to-book ratios, weaker profitability, and lower sensitivity of CEO turnover to firm performance. Consistent with a positive view, director busyness may signal director quality (Fama and Jensen, 1983). Ferris, Jagannathan, and Pritchard (2003) find a positive relationship between firm performance and additional directorships acquired by a director. Perry and Peyer (2005) find that when a firm has fewer agency problems the market positively responds to events in which executives with multiple directorships accept an additional outside directorship. Field, Lowry, and Mkrtchyan (2013) argue that busy directors are better advisors due to their experience and connections. They find that busy directors increase firm value in newly public firms, where advising is more important than monitoring.

By our definition, rookie directors are likely to be non-busy directors. This negative correlation between rookie directors and busy directors raises the possibility that the positive effect of rookie directors on firm performance is driven by the presence of fewer busy independent directors rather than more rookie independent directors on board. However, Table 2.2 shows that the correlation coefficient between Rookie director $(\%)_{t}$ and Busy director $(\%)_{t}$ is $-12.4 \%$. Therefore, the measure of rookie directors and busy directors is not mechanically correlated. Moreover, Giannetti, Liao, and Yu (2015) and Liang, Xu, and Jiraporn (2013) find that in China board busyness does not affect firm performance. We define Busy director $(0 / 1)_{t}$ as an independent director who has more than two directorships. We include Busy director $(\%)_{t}$ to control the busyness of boards for all firm level regressions. Consistent with Giannetti, Liao, and Yu (2015) and Liang, Xu, and Jiraporn (2013), we find that in 
Table 2.4 the coefficients associated with Busy director(\%) ${ }_{t}$ are negative but statistically no different than zero in explaining $R O S_{t}$ and $R O A_{t}$.

\subsubsection{Endogeneity tests on firm performance}

Our results may be subject to endogeneity issues. More specifically, an endogeneity issue may arise when rookie independent directors are not randomly assigned to firms. The presence of rookie independent directors in a firm may be driven by factors related to the demand of the firm for rookie independent directors or the willingness of rookie independent directors to join the firm. If some of these factors are correlated with firm performance and not properly controlled in performance regressions, the measures of rookie independent directors could be correlated with the error terms of performance regressions, leading to a biased OLS coefficient. For example, it is possible that firms experiencing reconstruction lose their experienced directors and appoint more rookie directors to their boards. If firms improve performance through reconstruction, there will be a positive relationship between the presence of rookie independent directors and firm performance. Besides, firms with good performance may have better corporate governance. Therefore, these firms are more likely to follow the regulation on term limits and do not allow their independent directors to serve beyond the term limits, leading to more rookie directors on their boards.

We apply several econometric methods to address endogeneity issues. The first method is a firm fixed effects regression. Firm fixed effects control for any time-invariant firmspecifics factors related to both firm performance and the presence of rookie independent directors. This method alleviates concerns relative to time-invariant omitted variables. Our results presented in Table 2.4 are robust to the firm fixed effects.

Another endogeneity concern is the possible reverse causality between the presence of rookie independent directors and firm performance. It is possible that changes in firm performance drive the appointment of rookie independent directors. To test this hypothesis, we examine whether firm characteristics at year $t-1$ predict the rookie independent director appointments at year $t$. In Table 2.8, we estimate linear probability regressions where the dependent variable Rookie appointment $(0 / 1)_{t}$ is a dummy variable that equals to 1 if a newly appointed independent director is a rookie director at year $t$ and 0 otherwise. We include both board features and firm characteristics at year $t-1$ as control variables. In columns (1) and (2), we find that the coefficients associated with $R O S_{t-1}$ and $R O A_{t-1}$ are not correlated with the dependent variable Rookie appointment $(0 / 1)_{t}$, suggesting the firm accounting return at year $t-1$ does not predict the rookie independent director appointments at year $t$. The above evidence suggests that our results are unlikely to be driven by reverse causality. 
To further mitigate the endogeneity concerns, we rerun our firm performance regressions with the IV-2SLS approach. To qualify as a valid instrument, a variable needs to be strongly correlated with the instrumented regressors (the validity requirement) but uncorrelated with the error term (the exclusion restriction). We construct two instrumental variables. The first instrument Retire director $(\%)_{t-1}$ is the mean value of the percentage of independent directors of other firms headquartered in the same city leaving their boards due to the term limits at year $t-1$. If the firms in a city retire more independent directors at year $t-1$, the demand for independent directors in this city would increase at year $t$. However, it is unlikely that the increase of demand for independent directors is satisfied by the limited supply of experienced directors from this city, leading the firms in this city to appoint more rookie independent directors. Therefore, Retire director $(\%)_{t-1}$ captures the local demand of rookie independent directors at year $t$. However, Retire director $(\%)_{t-1}$ is at the city average level and unlikely to directly influence individual firm performance.

Following the previous literature, we apply First-year director(\%) $t_{-1}$ as our second instrument variable (Knyazeva, Knyazeva, and Masulis, 2013, Liu, Miletkov, Wei, and Yang, 2015, Kang, Kim, and Low, 2016). First-year director $(\%)_{t-1}$ is the mean value of the percent of first-year directors of other firms headquartered in the same city at year $t-1$. The rationale behind First-year director $(\%)_{t-1}$ is that first-year directors from other firms in the same city at year $t-1$ are an important source of supply of rookie independent directors for firms at year $t$. First-year director $(\%)_{t-1}$ captures the local supply of rookie independent directors at year $t$. Similarly, First-year director $(\%)_{t-1}$ is a variable at the city level and unlikely to directly influence individual firm performance.

We confirm the suitability of our instruments by various identification tests reported in Table 2.9. In columns (1) and (2), we estimate the first-stage regressions with a linear probability model where the dependent variable is either Rookie director(\%)t or Rookie $\operatorname{board}(0 / 1)_{t}$. We find that our instruments Retire director $(\%)_{t-1}$ and First-year director $(\%)_{t-1}$ satisfy the validity requirement since they are positive and statistically significant at the

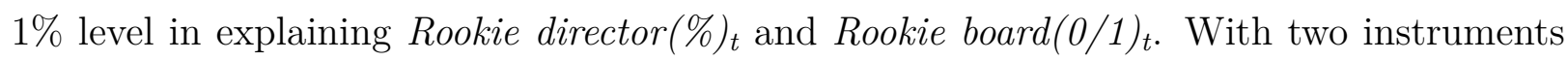
and only one endogenous regressor, we can conduct an over-identification test to examine whether the instruments satisfy the exclusion restriction. In columns (3) to (6), the Hansen J statistic for the over-identification test is reported and all p-values are over 0.1. The Hansen over-identification test fails to reject the hypothesis that our instruments are exogenous.

In columns (3) to (6) of Table 2.9, we estimate the second-stage regressions where the dependent variables are firm performance measures $R O S_{t}$ and $R O A_{t}$ and the indicator of rookie independent directors Rookie director $(\%)_{t}$ and Rookie board $(0 / 1)_{t}$ are replaced by their instrumented values from the first-stage regressions. In our IV-2SLS approach, firm fixed effects are included and all the control variables are lagged for one period. Results presented in columns (3) to (6) show that the coefficients associated with Rookie director(\%) 
and Rookie board $(0 / 1)_{t}$ are positive and statistically significant at the $5 \%$ level in explaining both $R O S_{t}$ and $R O A_{t}$. The IV-2SLS approach supports our findings that the presence of rookie independent directors improves the firm accounting performance.

\subsubsection{Alternative measure of ROS and ROA}

We conduct a robustness check on the measure of firm performance $R O S_{t}$ and $R O A_{t}$. In Table 2.10, we redefine $R O S_{t}$ and $R O A_{t}$ as EBITDA divided by sales and EBITDA divided by assets respectively. We rerun the regressions in Table 2.4 where the dependent variables are the redefined $R O S_{t}$ and $R O A_{t}$. The results are reported in Table 2.10. In columns (1) to (4), we find that the coefficients associated with Rookie director $(\%)_{t}$ and Rookie board $(0 / 1)_{t}$ are positive and statistically significant at the less than $5 \%$ level in explaining both $R O S_{t}$ and $R O A_{t}$. Therefore, our results are robust to the alternative measure of $R O S_{t}$ and $R O A_{t}$.

\subsubsection{Last-year director}

The career concern model suggests that directors near the retirement age are less motivated. In China, an independent director is allowed to serve a maximum of six years in a firm. Therefore, in China, directors could be less motivated when they are near their tenure limit (year 6 or year 7 ). To test whether previous results of board meeting attendance is driven by these less motivated directors, we include a variable Last-year director $(0 / 1)_{t}$ into board meeting attendance regression, where Last-year director $(0 / 1)_{t}$ is a dummy equals to 1 if an independent director is in his term limit (year 6 or year 7 ) and 0 otherwise.

Table 2.11 reports the results from regressions investigating the board meeting attendance when Last-year director $(0 / 1)_{t}$ is included. In columns (1) to (8), we find that the coefficients associated with Last-year director $(0 / 1)_{t}$ are positive and statistically significant at the less than $1 \%$ level in explaining Meeting absence $(0 / 1)_{t}$ and Meeting absence(\%). This result is consistent with the idea that directors serving in their term limit are less motivated. Moreover, we find that the coefficients associated with Rookie director $(0 / 1)_{t}$ are negative and statistically significant at the less than $5 \%$ level in explaining Meeting absence $(0 / 1)_{t}$ and Meeting absence(\%)t. Therefore, previous results of board meeting attendance are unchanged after Last-year director $(0 / 1)_{t}$ is included.

\subsection{Conclusion}

Rookie directors are an important source of labor for corporate boards. Despite the popularity of rookie directors on corporate boards, research on their impact on corporate governance and firm performance is limited in general and unstudied in China, where the ownership 
structure and governance issues differ from those in the U.S. ${ }^{26}$ Therefore, our study of rookie independent directors in China fills this gap.

Our study suggests that in China firms with rookie independent directors exhibit significantly better operating performance. This complements the existing finding that rookie independent directors increase firm values in the U.S.. However, the potential channel through which rookie independent directors improve the firm performance is different between the U.S. and China. In the U.S., rookie independent directors add to the firm values through their effective monitoring of CEOs. However, in China, rookie independent directors improve firm performance through their monitoring and management of tunneling to controlling shareholders.

We find that in China rookie independent directors improve firm performance most in non-state-owned firms. This is consistent with the prior literature finding that tunneling to controlling shareholders is more common in the non-state-owned firms than in state-owned firms. If the presence of rookie independent directors lessens tunneling to controlling shareholders, those firms more vulnerable to tunneling benefit more from the rookie independent directors.

\footnotetext{
${ }^{26} \mathrm{Kang}$, Kim, and Low (2016) is the only notable study on rookie directors and their influences on corporate governance and firm performance.
} 


\section{Bibliography}

Adams, Renée B., and Daniel Ferreira, 2008, Do directors perform for pay?, Journal of Accounting and Economics 46, 154-171.

Ahern, Kenneth R., and Amy K. Dittmar, 2012, The changing of the boards: The impact on firm valuation of mandated female board representation, The Quarterly Journal of Economics 127, 137-197.

Cai, Jie, Jacqueline L. Garner, and Ralph A. Walkling, 2009, Electing directors, The Journal of Finance 64, 2389-2421.

Chen, Zonghao, and Michael O'Connor Keefe, 2018, Board of director compensation in China: To pay or not to pay? How much to pay?, Emerging Markets Review 37, 66-82.

Chou, Hsin-I, Huimin Chung, and Xiangkang Yin, 2013, Attendance of board meetings and company performance: Evidence from Taiwan, Journal of Banking \&6 Finance 37, $4157-4171$.

Core, John E., Robert W. Holthausen, and David F. Larcker, 1999, Corporate governance, chief executive officer compensation, and firm performance, Journal of Financial Economics 51, 371-406.

Espen, Eckbo B., Nygaard Knut, and S. Thorburn Karin, 2018, Board gender-balancing and firm value, Available at SSRN.

Fama, Eugene F., and Michael C. Jensen, 1983, Separation of ownership and control, The Journal of Law 85 Economics 26, 301-325.

Ferris, Stephen P., Murali Jagannathan, and A. C. Pritchard, 2003, Too busy to mind the business? Monitoring by directors with multiple board appointments, The Journal of Finance 58, 1087-1111.

Fich, Eliezer M., and Anil Shivdasani, 2006, Are busy boards effective monitors?, The Journal of Finance 61, 689-724.

Field, Laura, Michelle Lowry, and Anahit Mkrtchyan, 2013, Are busy boards detrimental?, Journal of Financial Economics 109, 63-82.

Giannetti, Mariassunta, Guanmin Liao, and Xiaoyun Yu, 2015, The brain gain of corporate boards: Evidence from China, The Journal of Finance 70, 1629-1682.

He, Wen, and Jinhui Luo, 2018, Agency problems in firms with an even number of directors: Evidence from China, Journal of Banking \& Finance. 
Holmstrom, Bengt, 1982, Moral hazard in teams, The Bell Journal of Economics pp. 324340.

Jiang, Fuxiu, and Kenneth A. Kim, 2015, Corporate governance in China: A modern perspective, Journal of Corporate Finance 32, 190-216.

Jiang, Guohua, Charles MC Lee, and Heng Yue, 2010, Tunneling through intercorporate loans: The China experience, Journal of Financial Economics 98, 1-20.

Jiang, Wei, Hualin Wan, and Shan Zhao, 2015, Reputation concerns of independent directors: Evidence from individual director voting, The Review of Financial Studies 29, 655-696.

Kang, Jun-Koo, Jungmin Kim, and Angie Low, 2016, Rookie directors, Available at SSRN.

Knyazeva, Anzhela, Diana Knyazeva, and Ronald W. Masulis, 2013, The supply of corporate directors and board independence, The Review of Financial Studies 26, 1561-1605.

Liang, Qi, Pisun Xu, and Pornsit Jiraporn, 2013, Board characteristics and Chinese bank performance, Journal of Banking $\&$ Finance 37, 2953-2968.

Liu, Yu, Mihail K. Miletkov, Zuobao Wei, and Tina Yang, 2015, Board independence and firm performance in China, Journal of Corporate Finance 30, 223-244.

Liu, Yu, Zuobao Wei, and Feixue Xie, 2014, Do women directors improve firm performance in China?, Journal of Corporate Finance 28, 169-184.

Masulis, Ronald W., and Shawn Mobbs, 2014, Independent director incentives: Where do talented directors spend their limited time and energy?, Journal of Financial Economics 111, 406-429.

Masulis, Ronald W., Cong Wang, and Fei Xie, 2012, Globalizing the boardroom-the effects of foreign directors on corporate governance and firm performance, Journal of Accounting and Economics 53, 527-554.

Perry, Tod, and Urs Peyer, 2005, Board seat accumulation by executives: A shareholder's perspective, The Journal of Finance 60, 2083-2123.

Qian, Meijun, and Bernard Y. Yeung, 2015, Bank financing and corporate governance, Journal of Corporate Finance 32, 258-270.

Simonetta, Longhi, and Nandi Alita, 2015, A Practical Guide to Using Panel Data (SAGE Publications Ltd: London).

Yermack, David, 2005, Remuneration, retention, and reputation incentives for outside directors, The Journal of Finance 59, 2281-2308. 


\subsection{Tables}

Table 2.1: Summary statistics

This table provides the summary statistics for all variables. Table 2.12 provides all variable definitions. Panel A provides the summary statistics of director characteristics by director-year. Panel B provides the summary statistics of board characteristics by firm-year. Panel C provides the summary statistics of firm characteristics by firm-year. All monetary terms are denominated in Chinese Yuan (CNY).

\begin{tabular}{|c|c|c|c|c|c|c|}
\hline & Obs & Mean & SD & 25 th & Median & 75 th \\
\hline \multicolumn{7}{|c|}{ Panel A. Director characteristics (by director-year) } \\
\hline Rookie director $(0 / 1)_{t}$ & 42,608 & 0.257 & 0.437 & 0 & 0 & 1 \\
\hline $\operatorname{Woman}(0 / 1)$ & 42,608 & 0.146 & 0.354 & 0 & 0 & 0 \\
\hline Busy director $(0 / 1)_{t}$ & 42,608 & 0.260 & 0.439 & 0 & 0 & 1 \\
\hline Age $(\text { Ten years })_{t}$ & 42,608 & 5.303 & 0.972 & 4.6 & 5.1 & 6 \\
\hline Tenure in firm $(\text { Years })_{t}$ & 42,608 & 3.263 & 1.974 & 2 & 3 & 5 \\
\hline Number of directorshipst & 42,608 & 1.955 & 1.283 & 1 & 1 & 3 \\
\hline Director compensation(Thousands $C N Y)_{t}$ & 42,608 & 55.383 & 51.25 & 33.6 & 50 & 64.6 \\
\hline Political backgrounds $(0 / 1)_{t}$ & 42,608 & 0.398 & 0.489 & 0 & 0 & 1 \\
\hline Meeting frequency $y_{t}$ & 42,608 & 7.983 & 3.95 & 5 & 8 & 10 \\
\hline Meeting absence $(0 / 1)_{t}$ & 42,608 & 0.183 & 0.387 & 0 & 0 & 0 \\
\hline Meeting absence $(\%)_{t}$ & 42,608 & 0.034 & 0.094 & 0 & 0 & 0 \\
\hline Turnover $(0 / 1)_{t+1}$ & 37,508 & 0.143 & 0.35 & 0 & 0 & 0 \\
\hline Rookie appointment $(0 / 1)_{t}$ & 7,284 & 0.597 & 0.491 & 0 & 1 & 1 \\
\hline \multicolumn{7}{|l|}{ Panel B. Board characteristics (by firm-year) } \\
\hline Rookie director $(\%)_{t}$ & 12,433 & 0.256 & 0.302 & 0 & 0.2 & 0.4 \\
\hline Rookie board $(0 / 1)_{t}$ & 12,433 & 0.183 & 0.387 & 0 & 0 & 0 \\
\hline Women director $(\%)_{t}$ & 12,433 & 0.151 & 0.198 & 0 & 0 & 0.333 \\
\hline Busy director $(\%)_{t}$ & 12,433 & 0.260 & 0.252 & 0 & 0.250 & 0.333 \\
\hline Board size $_{t}$ & 12,433 & 8.843 & 1.728 & 8 & 9 & 9 \\
\hline Duality $_{t}$ & 12,433 & 0.238 & 0.426 & 0 & 0 & 0 \\
\hline Independent director $(\%)_{t}$ & 12,433 & 0.37 & 0.054 & 0.333 & 0.333 & 0.4 \\
\hline Retire director $(\%)_{t-1}$ & 10,234 & 0.08 & 0.111 & 0 & 0.055 & 0.111 \\
\hline First-year director $(\%)_{t-1}$ & 10,234 & 0.292 & 0.177 & 0.179 & 0.257 & 0.373 \\
\hline \multicolumn{7}{|l|}{ Panel C. Firm characteristics (by firm-year) } \\
\hline State-owned $(0 / 1)_{t}$ & 12,433 & 0.436 & 0.496 & 0 & 0 & 1 \\
\hline Largest shareholder $(\%)_{t}$ & 12,433 & 0.361 & 0.154 & 0.239 & 0.341 & 0.472 \\
\hline Firm age (Years $)_{t}$ & 12,433 & 15.004 & 5.274 & 11 & 15 & 19 \\
\hline Sales $(\text { Billions } C N Y)_{t}$ & 12,433 & 4.991 & 12.181 & 0.573 & 1.373 & 3.611 \\
\hline Total assets(Billions $C N Y)_{t}$ & 12,433 & 7.674 & 21.72 & 1.15 & 2.389 & 5.536 \\
\hline Book leverage $_{t}$ & 12,433 & 0.466 & 0.241 & 0.281 & 0.463 & 0.637 \\
\hline$R \& D(\%)_{t}$ & 12,433 & 0.003 & 0.014 & 0 & 0 & 0 \\
\hline $\operatorname{ORECTA}(\%)_{t}$ & 12,019 & 0.019 & 0.036 & 0.004 & 0.008 & 0.019 \\
\hline$R O A_{t}$ & 12,433 & 0.037 & 0.061 & 0.012 & 0.035 & 0.065 \\
\hline$R O S_{t}$ & 12,433 & 0.061 & 0.212 & 0.02 & 0.06 & 0.125 \\
\hline
\end{tabular}


Table 2.2: Cross correlations

This table provides the correlation matrix all variables from regression on firm performance. Table 2.12 provides all variable definitions. Superscripts $*, * *$ and ${ }^{* * *}$ denote significance at the $10 \%, 5 \%$ and $1 \%$ levels, respectively.

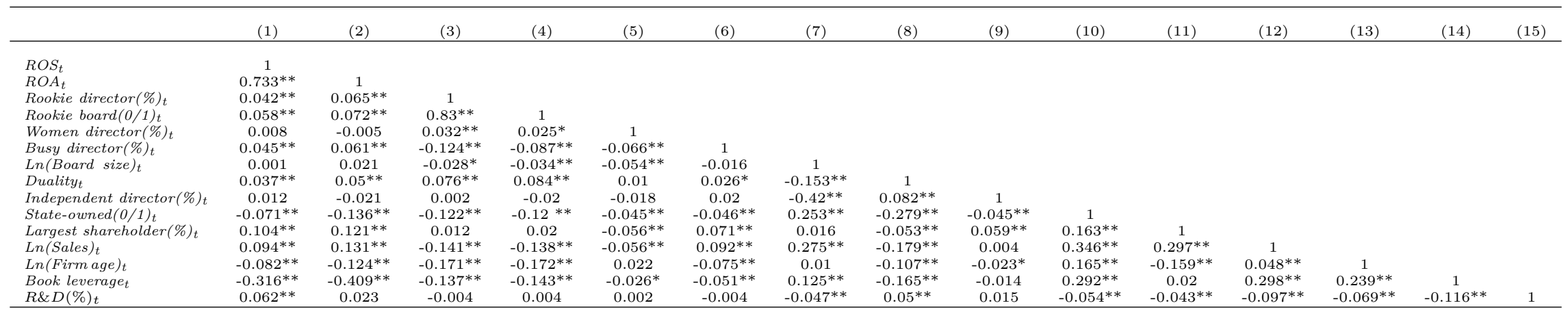


Table 2.3: Rookie directors and board meeting attendance

This table provides the board meeting attendance of rookie independent directors. In Columns (1) to (4), the dependent variable is Meeting absence $(0 / 1)_{t}$, a dummy variable that equals to 1 if an independent director absents any board meetings and 0 otherwise. In Column (5) to (8), the dependent variable is Meeting absence $(\%)_{t}$, the ratio of board meeting absences. The measure of rookie independent director is Rookie director $(0 / 1)_{t}$, a dummy variable that equals to 1 if an independent director has less than three years board experience and 0 otherwise. Table 2.12 provides all variable definitions. The regressions control for year, industry, firm, director and firm*year fixed effects. In parentheses are t-statistics based on standard errors adjusted for heteroskedasticity and firm clustering. Superscripts *, ** and *** denote significance at the $10 \%, 5 \%$ and $1 \%$ levels, respectively.

\begin{tabular}{|c|c|c|c|c|c|c|c|c|}
\hline \multirow[b]{2}{*}{ Explanatory variables } & \multicolumn{4}{|c|}{ Meeting absence $(0 / 1)_{t}$} & \multicolumn{4}{|c|}{ Meeting absence $(\%)_{t}$} \\
\hline & (1) & $(2)$ & $(3)$ & $(4)$ & $(5)$ & $(6)$ & $(7)$ & $(8)$ \\
\hline Rookie director $(0 / 1)_{t}$ & $\begin{array}{c}-0.012^{* *} \\
(-2.42)\end{array}$ & $\begin{array}{c}-0.016^{* * *} \\
(-3.10)\end{array}$ & $\begin{array}{c}-0.016^{* *} \\
(-2.42)\end{array}$ & $\begin{array}{c}-0.017^{* * *} \\
(-2.84)\end{array}$ & $\begin{array}{c}-0.005^{* * *} \\
(-3.37)\end{array}$ & $\begin{array}{c}-0.006^{* * *} \\
(-4.12)\end{array}$ & $\begin{array}{c}-0.005^{* *} \\
(-2.57)\end{array}$ & $\begin{array}{c}-0.006^{* * *} \\
(-3.29)\end{array}$ \\
\hline $\operatorname{Woman}(0 / 1)$ & $\begin{array}{l}-0.006 \\
(-1.18)\end{array}$ & $\begin{array}{l}-0.008 \\
(-1.42)\end{array}$ & & $\begin{array}{c}-0.014^{* *} \\
(-2.44)\end{array}$ & $\begin{array}{l}-0.001 \\
(-0.79)\end{array}$ & $\begin{array}{l}-0.001 \\
(-0.79)\end{array}$ & & $\begin{array}{l}-0.002 \\
(-0.95)\end{array}$ \\
\hline $\operatorname{Age}(\text { Ten years })_{t}$ & $\begin{array}{c}-0.006^{* * *} \\
(-2.93)\end{array}$ & $\begin{array}{c}-0.006^{* * *} \\
(-2.88)\end{array}$ & $\begin{array}{l}0.009 \\
(0.09)\end{array}$ & $\begin{array}{c}-0.007 * * * \\
(-2.95)\end{array}$ & $\begin{array}{c}-0.001^{* *} \\
(-2.06)\end{array}$ & $\begin{array}{c}-0.001^{*} \\
(-1.90)\end{array}$ & $\begin{array}{l}0.020 \\
(0.58)\end{array}$ & $\begin{array}{c}-0.001^{*} \\
(-1.70)\end{array}$ \\
\hline Tenure in firm(Years) $t$ & $\begin{array}{c}0.006^{* * *} \\
(4.88)\end{array}$ & $\begin{array}{c}0.005^{* * *} \\
(4.08)\end{array}$ & $\begin{array}{c}0.009^{* * *} \\
(5.87)\end{array}$ & $\begin{array}{c}0.006^{* * *} \\
(4.13)\end{array}$ & $\begin{array}{c}0.002^{* * *} \\
(4.55)\end{array}$ & $\begin{array}{c}0.002^{* * *} \\
(4.69)\end{array}$ & $\begin{array}{c}0.002^{* * *} \\
(4.90)\end{array}$ & $\begin{array}{c}0.003^{* * *} \\
(4.84)\end{array}$ \\
\hline Busy director $(0 / 1)_{t}$ & $\begin{array}{l}0.002 \\
(0.45)\end{array}$ & $\begin{array}{l}0.000 \\
(0.07)\end{array}$ & $\begin{array}{l}-0.005 \\
(-0.65)\end{array}$ & $\begin{array}{l}-0.002 \\
(-0.37)\end{array}$ & $\begin{array}{c}-0.003^{* *} \\
(-2.19)\end{array}$ & $\begin{array}{c}-0.003^{* *} \\
(-2.12)\end{array}$ & $\begin{array}{c}-0.004^{* *} \\
(-2.38)\end{array}$ & $\begin{array}{c}-0.004^{* *} \\
(-2.02)\end{array}$ \\
\hline Ln(Director compensation $)_{t}$ & $\begin{array}{c}0.002^{* * *} \\
(3.46)\end{array}$ & $\begin{array}{l}0.001 \\
(0.79)\end{array}$ & $\begin{array}{c}0.003^{* * *} \\
(3.47)\end{array}$ & $\begin{array}{l}0.001 \\
(1.14)\end{array}$ & $\begin{array}{c}-0.001^{* *} \\
(-2.04)\end{array}$ & $\begin{array}{c}-0.001^{* *} \\
(-2.55)\end{array}$ & $\begin{array}{l}-0.000 \\
(-1.13)\end{array}$ & $\begin{array}{l}-0.000 \\
(-0.61)\end{array}$ \\
\hline Political backgrounds $(0 / 1)_{t}$ & $\begin{array}{l}0.004 \\
(1.07)\end{array}$ & $\begin{array}{l}0.003 \\
(0.70)\end{array}$ & $\begin{array}{c}-0.221^{* * *} \\
(-3.60)\end{array}$ & $\begin{array}{l}0.003 \\
(0.70)\end{array}$ & $\begin{array}{l}0.001 \\
(0.67)\end{array}$ & $\begin{array}{l}0.000 \\
(0.28)\end{array}$ & $\begin{array}{l}-0.019 \\
(-0.72)\end{array}$ & $\begin{array}{l}0.000 \\
(0.26)\end{array}$ \\
\hline Meeting frequency $y_{t}$ & $\begin{array}{c}0.008^{* * *} \\
(15.78)\end{array}$ & $\begin{array}{c}0.011^{* * *} \\
(17.61)\end{array}$ & $\begin{array}{c}0.009^{* * *} \\
(15.01)\end{array}$ & $\begin{array}{c}0.011^{* * *} \\
(10.37)\end{array}$ & $\begin{array}{c}-0.002^{* * *} \\
(-7.74)\end{array}$ & $\begin{array}{c}-0.001^{* * *} \\
(-6.59)\end{array}$ & $\begin{array}{c}-0.001^{* * *} \\
(-7.07)\end{array}$ & $\begin{array}{c}-0.002^{* * *} \\
(-4.55)\end{array}$ \\
\hline Ln(Board size $)_{t}$ & $\begin{array}{c}0.164^{* * *} \\
(15.34)\end{array}$ & $\begin{array}{l}-0.000 \\
(-0.00)\end{array}$ & $\begin{array}{c}0.083^{* * *} \\
(4.76)\end{array}$ & & $\begin{array}{c}0.038^{* * *} \\
(8.03)\end{array}$ & $\begin{array}{l}0.008 \\
(1.12)\end{array}$ & $\begin{array}{c}0.020^{* * *} \\
(3.93)\end{array}$ & \\
\hline Duality $_{t}$ & $\begin{array}{l}-0.002 \\
(-0.36)\end{array}$ & $\begin{array}{l}0.001 \\
(0.12)\end{array}$ & $\begin{array}{l}-0.003 \\
(-0.46)\end{array}$ & & $\begin{array}{l}0.002 \\
(0.92)\end{array}$ & $\begin{array}{l}0.003 \\
(1.42)\end{array}$ & $\begin{array}{l}0.001 \\
(0.60)\end{array}$ & \\
\hline Independent director $(\%)_{t}$ & $\begin{array}{c}0.142^{* * *} \\
(3.94)\end{array}$ & $\begin{array}{l}-0.109 \\
(-1.64)\end{array}$ & $\begin{array}{l}0.076 \\
(1.39)\end{array}$ & & $\begin{array}{c}0.048^{* * *} \\
(3.02)\end{array}$ & $\begin{array}{l}-0.015 \\
(-0.70)\end{array}$ & $\begin{array}{c}0.035^{* *} \\
(2.25)\end{array}$ & \\
\hline State-owned $(0 / 1)_{t}$ & $\begin{array}{c}0.035^{* * *} \\
(8.29)\end{array}$ & $\begin{array}{l}0.022 \\
(1.35)\end{array}$ & $\begin{array}{c}0.037^{* * *} \\
(4.77)\end{array}$ & & $\begin{array}{c}0.007^{* * *} \\
(4.16)\end{array}$ & $\begin{array}{l}0.006 \\
(0.87)\end{array}$ & $\begin{array}{c}0.008^{* * *} \\
(3.47)\end{array}$ & \\
\hline Largest shareholder $(\%)_{t}$ & $\begin{array}{l}-0.018 \\
(-1.40)\end{array}$ & $\begin{array}{l}-0.052 \\
(-1.29)\end{array}$ & $\begin{array}{c}-0.052^{* *} \\
(-2.38)\end{array}$ & & $\begin{array}{l}-0.007 \\
(-1.20)\end{array}$ & $\begin{array}{l}-0.008 \\
(-0.68)\end{array}$ & $\begin{array}{c}-0.014^{* *} \\
(-2.37)\end{array}$ & \\
\hline $\operatorname{Ln}(\text { Sales })_{t}$ & $\begin{array}{l}-0.000 \\
(-0.09)\end{array}$ & $\begin{array}{l}-0.004 \\
(-0.91)\end{array}$ & $\begin{array}{c}-0.005^{*} \\
(-1.76)\end{array}$ & & $\begin{array}{l}0.000 \\
(0.49)\end{array}$ & $\begin{array}{l}-0.001 \\
(-0.39)\end{array}$ & $\begin{array}{l}-0.000 \\
(-0.63)\end{array}$ & \\
\hline Book leverage $_{t}$ & $\begin{array}{c}0.038^{* * *} \\
(3.83)\end{array}$ & $\begin{array}{l}-0.001 \\
(-0.06)\end{array}$ & $\begin{array}{c}0.061^{* * *} \\
(3.95)\end{array}$ & & $\begin{array}{c}0.011^{* *} \\
(2.30)\end{array}$ & $\begin{array}{l}0.001 \\
(0.22)\end{array}$ & $\begin{array}{c}0.012^{* *} \\
(2.57)\end{array}$ & \\
\hline$R O A_{t}$ & $\begin{array}{l}-0.004 \\
(-0.11)\end{array}$ & $\begin{array}{l}0.020 \\
(0.43)\end{array}$ & $\begin{array}{c}0.086^{*} \\
(1.88)\end{array}$ & & $\begin{array}{l}-0.005 \\
(-0.41)\end{array}$ & $\begin{array}{l}0.000 \\
(0.03)\end{array}$ & $\begin{array}{l}0.014 \\
(1.00)\end{array}$ & \\
\hline Year effects & Yes & Yes & Yes & No & Yes & Yes & Yes & No \\
\hline Industry effects & Yes & No & Yes & No & Yes & No & Yes & No \\
\hline Firm effects & No & Yes & No & No & No & Yes & No & No \\
\hline Director effects & No & No & Yes & No & No & No & Yes & No \\
\hline Firm*year effects & No & No & No & Yes & No & No & No & Yes \\
\hline$R^{2}$ & 0.030 & 0.185 & 0.345 & 0.413 & 0.022 & 0.151 & 0.364 & 0.354 \\
\hline Observations & 42,608 & 42,608 & 42,608 & 42,608 & 42,608 & 42,608 & 42,608 & 42,608 \\
\hline
\end{tabular}


Table 2.4: Rookie directors and firm performance

This table provides the results of rookie directors and firm performance. We use both Rookie director $(\%)_{t}$ and Rookie board $(0 / 1)_{t}$ to proxy the presence of rookie independent directors. Rookie director $(\%)_{t}$ is the ratio of rookie independent directors. Rookie board $(0 / 1)_{t}$ is a dummy variable that equals to 1 if the majority of independent directors are rookies. In Column (1) and (2), the firm performance is measured by $R O S_{t}$. In Column (3) and (4), the firm performance is measured by $R O A_{t}$. Table 2.12 provides all variable definitions. All regressions control for year and firm fixed effects. In parentheses are t-statistics based on standard errors adjusted for heteroskedasticity and firm clustering. Superscripts *, ** and *** denote significance at the $10 \%$, $5 \%$ and $1 \%$ levels, respectively.

\begin{tabular}{|c|c|c|c|c|}
\hline \multirow[b]{2}{*}{ Explanatory variables } & \multicolumn{2}{|c|}{ ROS $(\text { Net Income } / \text { Sales })_{t}$} & \multicolumn{2}{|c|}{$R O A(\text { Net Income } / \text { Assets })_{t}$} \\
\hline & (1) & $(2)$ & $(3)$ & $(4)$ \\
\hline Rookie director $(\%)_{t}$ & $\begin{array}{c}0.016^{* *} \\
(2.41)\end{array}$ & & $\begin{array}{c}0.005^{* * *} \\
(3.04)\end{array}$ & \\
\hline Rookie board $(0 / 1)_{t}$ & & $\begin{array}{c}0.016^{* * *} \\
(3.23)\end{array}$ & & $\begin{array}{c}0.004^{* * *} \\
(2.68)\end{array}$ \\
\hline Women director $(\%)_{t}$ & $\begin{array}{l}0.037^{*} \\
(1.81)\end{array}$ & $\begin{array}{c}0.037^{*} \\
(1.82)\end{array}$ & $\begin{array}{l}0.008 \\
(1.51)\end{array}$ & $\begin{array}{l}0.008 \\
(1.53)\end{array}$ \\
\hline Busy director $(\%)_{t}$ & $\begin{array}{l}-0.016 \\
(-1.47)\end{array}$ & $\begin{array}{l}-0.017 \\
(-1.55)\end{array}$ & $\begin{array}{l}-0.003 \\
(-1.23)\end{array}$ & $\begin{array}{l}-0.004 \\
(-1.35)\end{array}$ \\
\hline Ln(Board size $)_{t}$ & $\begin{array}{l}-0.026 \\
(-0.81)\end{array}$ & $\begin{array}{l}-0.025 \\
(-0.80)\end{array}$ & $\begin{array}{l}-0.005 \\
(-0.65)\end{array}$ & $\begin{array}{l}-0.005 \\
(-0.62)\end{array}$ \\
\hline Duality $_{t}$ & $\begin{array}{l}0.007 \\
(0.75)\end{array}$ & $\begin{array}{l}0.007 \\
(0.72)\end{array}$ & $\begin{array}{l}0.003 \\
(1.42)\end{array}$ & $\begin{array}{l}0.003 \\
(1.41)\end{array}$ \\
\hline Independent director $(\%)_{t}$ & $\begin{array}{l}0.008 \\
(0.11)\end{array}$ & $\begin{array}{l}0.011 \\
(0.16)\end{array}$ & $\begin{array}{l}-0.017 \\
(-0.95)\end{array}$ & $\begin{array}{l}-0.016 \\
(-0.90)\end{array}$ \\
\hline State-owned $(0 / 1)_{t}$ & $\begin{array}{l}-0.048 \\
(-1.62)\end{array}$ & $\begin{array}{l}-0.049 \\
(-1.64)\end{array}$ & $\begin{array}{c}-0.026^{* * *} \\
(-3.92)\end{array}$ & $\begin{array}{c}-0.026^{* * *} \\
(-3.96)\end{array}$ \\
\hline Largest shareholder $(\%)_{t}$ & $\begin{array}{l}0.126^{*} \\
(1.88)\end{array}$ & $\begin{array}{c}0.125^{*} \\
(1.86)\end{array}$ & $\begin{array}{c}0.041^{* *} \\
(2.35)\end{array}$ & $\begin{array}{c}0.041^{* *} \\
(2.35)\end{array}$ \\
\hline $\operatorname{Ln}\left(\right.$ Sales $_{t}$ & $\begin{array}{c}0.071^{* * *} \\
(5.80)\end{array}$ & $\begin{array}{c}0.071^{* * *} \\
(5.80)\end{array}$ & $\begin{array}{c}0.022^{* * *} \\
(10.95)\end{array}$ & $\begin{array}{c}0.022^{* * *} \\
(10.95)\end{array}$ \\
\hline $\operatorname{Ln}(\text { Firm age })_{t}$ & $\begin{array}{c}-0.006^{* * *} \\
(-2.89)\end{array}$ & $\begin{array}{c}-0.006^{* * *} \\
(-2.90)\end{array}$ & $\begin{array}{c}-0.003^{* * *} \\
(-5.79)\end{array}$ & $\begin{array}{c}-0.003^{* * *} \\
(-5.90)\end{array}$ \\
\hline Book leverage $_{t}$ & $\begin{array}{c}-0.453^{* * *} \\
(-10.63)\end{array}$ & $\begin{array}{c}-0.452^{* * *} \\
(-10.62)\end{array}$ & $\begin{array}{c}-0.139^{* * *} \\
(-15.84)\end{array}$ & $\begin{array}{c}-0.139^{* * *} \\
(-15.84)\end{array}$ \\
\hline$R \& D(\%)_{t}$ & $\begin{array}{l}-0.196 \\
(-1.18)\end{array}$ & $\begin{array}{l}-0.192 \\
(-1.16)\end{array}$ & $\begin{array}{c}-0.110^{* *} \\
(-1.97)\end{array}$ & $\begin{array}{c}-0.111^{* *} \\
(-1.99)\end{array}$ \\
\hline Year effects & Yes & Yes & Yes & Yes \\
\hline Firm effects & Yes & Yes & Yes & Yes \\
\hline$R^{2}$ & 0.117 & 0.117 & 0.181 & 0.181 \\
\hline Observations & 12,433 & 12,433 & 12,433 & 12,433 \\
\hline
\end{tabular}


Table 2.5: Rookie directors and tunneling

This table provides the results of rookie directors on the tunneling behavior of controlling shareholders. We use both Rookie director $(\%)_{t}$ and Rookie board $(0 / 1)_{t}$ to proxy the presence of rookie independent directors. Rookie director $(\%)_{t}$ is the ratio of rookie independent directors. Rookie board $(0 / 1)_{t}$ is a dummy variable that equals to one if the majority of independent directors are rookies. We measure the tunneling behavior of controlling shareholders by $\operatorname{ORECTA}(\%)_{t}$, which is other receivables scaled by total assets. Table 2.12 provides all variable definitions. The regressions control for year, industry and firm fixed effects. In parentheses are t-statistics based on standard errors adjusted for heteroskedasticity and firm clustering. Superscripts $*,{ }^{* *}$ and ${ }^{* * *}$ denote significance at the $10 \%, 5 \%$ and $1 \%$ levels, respectively.

\begin{tabular}{|c|c|c|c|c|}
\hline \multirow[b]{2}{*}{ Explanatory variables } & \multicolumn{4}{|c|}{$\operatorname{ORECTA}(\%)_{t}$} \\
\hline & (1) & $(2)$ & (3) & (4) \\
\hline Rookie director $(\%)_{t}$ & $\begin{array}{c}-0.003^{* *} \\
(-2.03)\end{array}$ & & $\begin{array}{c}-0.003^{* *} \\
(-2.17)\end{array}$ & \\
\hline Rookie board $(0 / 1)_{t}$ & & $\begin{array}{c}-0.002^{* *} \\
(-2.18)\end{array}$ & & $\begin{array}{c}-0.003^{* * * *} \\
(-2.80)\end{array}$ \\
\hline Women director $(\%)_{t}$ & $\begin{array}{l}-0.002 \\
(-1.07)\end{array}$ & $\begin{array}{l}-0.002 \\
(-1.09)\end{array}$ & $\begin{array}{l}-0.001 \\
(-0.30)\end{array}$ & $\begin{array}{l}-0.001 \\
(-0.31)\end{array}$ \\
\hline Busy director $(\%)_{t}$ & $\begin{array}{l}-0.001 \\
(-0.91)\end{array}$ & $\begin{array}{l}-0.001 \\
(-0.82)\end{array}$ & $\begin{array}{l}0.000 \\
(0.28)\end{array}$ & $\begin{array}{l}0.001 \\
(0.37)\end{array}$ \\
\hline Ln $(\text { Board size })_{t}$ & $\begin{array}{l}-0.002 \\
(-0.64)\end{array}$ & $\begin{array}{l}-0.002 \\
(-0.69)\end{array}$ & $\begin{array}{l}-0.004 \\
(-0.82)\end{array}$ & $\begin{array}{l}-0.004 \\
(-0.85)\end{array}$ \\
\hline Duality $_{t}$ & $\begin{array}{l}-0.001 \\
(-0.95)\end{array}$ & $\begin{array}{l}-0.001 \\
(-0.93)\end{array}$ & $\begin{array}{l}0.001 \\
(0.47)\end{array}$ & $\begin{array}{l}0.001 \\
(0.49)\end{array}$ \\
\hline Independent director $(\%)_{t}$ & $\begin{array}{l}0.004 \\
(0.53)\end{array}$ & $\begin{array}{l}0.004 \\
(0.47)\end{array}$ & $\begin{array}{l}0.005 \\
(0.40)\end{array}$ & $\begin{array}{l}0.005 \\
(0.36)\end{array}$ \\
\hline State-owned $(0 / 1)_{t}$ & $\begin{array}{c}-0.003^{* *} \\
(-2.08)\end{array}$ & $\begin{array}{c}-0.002^{* *} \\
(-2.06)\end{array}$ & $\begin{array}{l}-0.006 \\
(-0.73)\end{array}$ & $\begin{array}{l}-0.006 \\
(-0.71)\end{array}$ \\
\hline Largest shareholder $(\%)_{t}$ & $\begin{array}{c}-0.017^{* * * *} \\
(-5.28)\end{array}$ & $\begin{array}{c}-0.017^{* * *} \\
(-5.26)\end{array}$ & $\begin{array}{l}-0.019 \\
(-1.34)\end{array}$ & $\begin{array}{l}-0.019 \\
(-1.33)\end{array}$ \\
\hline Ln $(\text { Sales })_{t}$ & $\begin{array}{c}-0.003^{* * *} \\
(-4.43)\end{array}$ & $\begin{array}{c}-0.003^{* * *} \\
(-4.44)\end{array}$ & $\begin{array}{c}-0.007^{* * *} \\
(-3.68)\end{array}$ & $\begin{array}{c}-0.007^{* * * *} \\
(-3.68)\end{array}$ \\
\hline$R O A_{t-1}$ & $\begin{array}{c}-0.073^{* * *} \\
(-7.24)\end{array}$ & $\begin{array}{c}-0.073^{\text {*** }} \\
(-7.21)\end{array}$ & $\begin{array}{c}-0.040^{* * *} \\
(-2.88)\end{array}$ & $\begin{array}{c}-0.040 \text { *** } \\
(-2.87)\end{array}$ \\
\hline Year effects & Yes & Yes & Yes & Yes \\
\hline Industry effects & Yes & Yes & No & No \\
\hline Firm effects & No & No & Yes & Yes \\
\hline$R^{2}$ & 0.090 & 0.090 & 0.528 & 0.528 \\
\hline Observations & 12,019 & 12,019 & 12,019 & 12,019 \\
\hline
\end{tabular}


Table 2.6: Rookie directors and director turnover

This table provides the results of meeting attendance on director turnover. We measure the director turnover by Turnover $(0 / 1)_{t+1}$, a dummy variable that equals to 1 if independent directors lose one of their directorships at year $t+1$ and 0 otherwise. The board meeting attendance measures is Meeting absence $(0 / 1)_{t}$, a dummy variable that equals to 1 if an independent director absents any board meetings and 0 otherwise. The measure of rookie independent director is Rookie director $(0 / 1)_{t}$, a dummy variable that equal to 1 if an independent director has less than three years' board experience and 0 otherwise. Table 2.12 provides all variable definitions. The regressions control for year, industry, firm, director and firm*year fixed effects. In parentheses are t-statistics based on standard errors adjusted for heteroskedasticity and firm clustering. Superscripts *,** and $* * *$ denote significance at the $10 \%, 5 \%$ and $1 \%$ levels, respectively.

\begin{tabular}{|c|c|c|c|c|}
\hline \multirow[b]{2}{*}{ Explanatory variables } & \multicolumn{4}{|c|}{ Turnover $(0 / 1)_{t+1}$} \\
\hline & (1) & $(2)$ & & (4) \\
\hline Rookie director $(0 / 1)_{t}$ & $\begin{array}{c}-0.009^{* *} \\
(-2.22)\end{array}$ & $\begin{array}{c}-0.010^{* *} \\
(-2.21)\end{array}$ & $\begin{array}{c}0.012^{* *} \\
(2.23)\end{array}$ & $\begin{array}{c}-0.028^{* * *} \\
(-5.12)\end{array}$ \\
\hline Meeting absence $(0 / 1)_{t}$ & $\begin{array}{c}0.030^{* * *} \\
(5.91)\end{array}$ & $\begin{array}{c}0.031^{* * * *} \\
(5.79)\end{array}$ & $\begin{array}{c}0.025^{* * *} \\
(4.45)\end{array}$ & $\begin{array}{c}0.042^{* * *} \\
(7.55)\end{array}$ \\
\hline Rookie director $(0 / 1)_{t}{ }^{*}$ Meeting absence $(0 / 1)_{t}$ & $\begin{array}{c}0.031^{* * *} \\
(3.71)\end{array}$ & $\begin{array}{c}0.034^{* * *} \\
(3.91)\end{array}$ & $\begin{array}{l}0.015^{*} \\
(1.71)\end{array}$ & $\begin{array}{c}0.044^{* * *} \\
(4.48)\end{array}$ \\
\hline $\operatorname{Woman}(0 / 1)$ & $\begin{array}{c}-0.015^{* * *} \\
(-3.26)\end{array}$ & $\begin{array}{c}-0.016^{* * *} \\
(-3.07)\end{array}$ & & $\begin{array}{c}-0.017^{* * *} \\
(-3.49)\end{array}$ \\
\hline Age $(\text { Ten years })_{t}$ & $\begin{array}{c}0.004^{*} \\
(1.92)\end{array}$ & $\begin{array}{c}0.004^{* *} \\
(2.00)\end{array}$ & $\begin{array}{l}-0.122 \\
(-0.91)\end{array}$ & $\begin{array}{c}0.005^{* *} \\
(2.53)\end{array}$ \\
\hline Tenure in firm(Years $)_{t}$ & $\begin{array}{c}0.070^{* * *} \\
(51.08)\end{array}$ & $\begin{array}{c}0.092^{* * *} \\
(62.48)\end{array}$ & $\begin{array}{c}0.085^{* * *} \\
(43.99)\end{array}$ & $\begin{array}{c}0.095^{* * *} \\
(57.38)\end{array}$ \\
\hline Busy director $(0 / 1)_{t}$ & $\begin{array}{c}-0.075^{* * *} \\
(-19.64)\end{array}$ & $\begin{array}{c}-0.098^{* * *} \\
(-20.97)\end{array}$ & $\begin{array}{c}-0.113^{* * *} \\
(-17.45)\end{array}$ & $\begin{array}{c}-0.087^{* * *} \\
(-18.24)\end{array}$ \\
\hline${ }^{L n}(\text { Director compensation })_{t}$ & $\begin{array}{c}-0.004^{* * *} \\
(-5.35)\end{array}$ & $\begin{array}{c}-0.003^{* * *} \\
(-3.21)\end{array}$ & $\begin{array}{c}-0.006^{* * *} \\
(-5.87)\end{array}$ & $\begin{array}{c}0.013^{* * *} \\
(8.69)\end{array}$ \\
\hline Political backgrounds $(0 / 1)_{t}$ & $\begin{array}{l}-0.004 \\
(-1.07)\end{array}$ & $\begin{array}{l}-0.004 \\
(-0.86)\end{array}$ & $\begin{array}{l}0.083 \\
(0.82)\end{array}$ & $\begin{array}{l}-0.003 \\
(-0.95)\end{array}$ \\
\hline $\operatorname{Ln}(\text { Meeting frequency })_{t}$ & $\begin{array}{c}-0.125^{* * *} \\
(-34.02)\end{array}$ & $\begin{array}{c}-0.170 * * * \\
(-39.19)\end{array}$ & $\begin{array}{c}-0.157^{* * *} \\
(-36.74)\end{array}$ & $\begin{array}{c}-0.163^{* * *} \\
(-22.58)\end{array}$ \\
\hline Ln(Board size $)_{t}$ & $\begin{array}{c}-0.053^{* * *} \\
(-5.40)\end{array}$ & $\begin{array}{c}-0.124^{* * *} \\
(-4.58)\end{array}$ & $\begin{array}{c}-0.120^{* * *} \\
(-6.56)\end{array}$ & \\
\hline Duality $_{t}$ & $\begin{array}{l}0.005 \\
(1.32)\end{array}$ & $\begin{array}{l}0.002 \\
(0.28)\end{array}$ & $\begin{array}{l}-0.007 \\
(-1.10)\end{array}$ & \\
\hline Independent director $(\%)_{t}$ & $\begin{array}{c}-0.148^{* * *} \\
(-4.79)\end{array}$ & $\begin{array}{c}-0.211^{* * *} \\
(-2.95)\end{array}$ & $\begin{array}{c}-0.279^{* * *} \\
(-5.24)\end{array}$ & \\
\hline State-owned $(0 / 1)_{t}$ & $\begin{array}{c}-0.034^{* * * *} \\
(-8.75)\end{array}$ & $\begin{array}{l}0.006 \\
(0.33)\end{array}$ & $\begin{array}{c}-0.036^{* * *} \\
(-4.76)\end{array}$ & \\
\hline Largest shareholder $(\%)_{t}$ & $\begin{array}{c}0.030^{* * *} \\
(2.74)\end{array}$ & $\begin{array}{l}-0.066 \\
(-1.55)\end{array}$ & $\begin{array}{l}0.025 \\
(1.21)\end{array}$ & \\
\hline $\operatorname{Ln}(\text { Sales })_{t}$ & $\begin{array}{l}0.002 \\
(1.42)\end{array}$ & $\begin{array}{l}0.001 \\
(0.18)\end{array}$ & $\begin{array}{l}0.003 \\
(1.08)\end{array}$ & \\
\hline Book leverage $_{t}$ & $\begin{array}{c}-0.036^{* * *} \\
(-4.05)\end{array}$ & $\begin{array}{c}-0.043^{* *} \\
(-2.16)\end{array}$ & $\begin{array}{c}-0.031^{* *} \\
(-2.23)\end{array}$ & \\
\hline$R O A_{t}$ & $\begin{array}{c}-0.147^{* * *} \\
(-4.34)\end{array}$ & $\begin{array}{l}-0.068 \\
(-1.53)\end{array}$ & $\begin{array}{c}-0.153^{* * *} \\
(-3.61)\end{array}$ & \\
\hline Year effects & Yes & Yes & Yes & No \\
\hline Industry effects & Yes & No & Yes & No \\
\hline Firm effects & No & Yes & No & No \\
\hline Director effects & No & No & Yes & No \\
\hline Firm*year effects & No & No & No & Yes \\
\hline$R^{2}$ & 0.173 & 0.270 & 0.442 & 0.589 \\
\hline Observations & 37,508 & 37,508 & 37,508 & 37,508 \\
\hline
\end{tabular}


Table 2.7: Rookie directors and firm performance (state-owned vs non-state-owned)

This table differentiates the effects of rookie independent directors on firm performance between state-owned and non-state-owned firms. We use both Rookie director $(\%)_{t}$ and Rookie board $(0 / 1)_{t}$ to proxy the presence of rookie independent directors. Rookie director $(\%)_{t}$ is the ratio of rookie independent directors. Rookie board $(0 / 1)_{t}$ is a dummy variable that equals to 1 if the majority of independent directors are rookies. In Columns (1) and (2), the firm performance is measured by $R O S_{t}$. In Columns (3) and (4), the firm performance is measured by $R O A_{t}$. The interaction terms between Rookie director $(\%)_{t}$ and State-owned $(0 / 1)_{t}$ or Rookie board $(0 / 1)_{t}$ and State-owned $(0 / 1)_{t}$ differentiate the marginal effects of rookie independent directors on firm performance between state-owned and non-state-owned firms. Table 2.12 provides all variable definitions. All regressions control for year and firm fixed effects. In parentheses are t-statistics based on standard errors adjusted for heteroskedasticity and firm clustering. Superscripts *, ** and ${ }^{* * *}$ denote significance at the $10 \%, 5 \%$ and $1 \%$ levels, respectively.

\begin{tabular}{|c|c|c|c|c|}
\hline Explanatory variables & \multicolumn{2}{|c|}{$\begin{array}{l}\text { ROS }(\text { Net Income } / \text { Sales })_{t} \\
(1)\end{array}$} & \multicolumn{2}{|c|}{$R O A(\text { Net Income } / \text { Assets })_{t}$} \\
\hline State-owned $(0 / 1)_{t}$ & $\begin{array}{l}-0.042 \\
(-1.40)\end{array}$ & $\begin{array}{l}-0.045 \\
(-1.51)\end{array}$ & $\begin{array}{c}-0.023^{* * *} \\
(-3.47)\end{array}$ & $\begin{array}{c}-0.025^{* * *} \\
(-3.70)\end{array}$ \\
\hline Rookie director $(\%)_{t}$ & $\begin{array}{c}0.025^{* * *} \\
(2.96)\end{array}$ & & $\begin{array}{c}0.009^{* * *} \\
(4.35)\end{array}$ & \\
\hline Rookie board $(0 / 1)_{t}$ & & $\begin{array}{c}0.024^{* * *} \\
(4.04)\end{array}$ & & $\begin{array}{c}0.007^{* * *} \\
(4.35)\end{array}$ \\
\hline State-owned $(0 / 1)_{t}{ }^{*}$ Rookie director $(\%)_{t}$ & $\begin{array}{c}-0.025^{* *} \\
(-2.08)\end{array}$ & & $\begin{array}{c}-0.011^{* * *} \\
(-3.45)\end{array}$ & \\
\hline State-owned $(0 / 1)_{t}{ }^{*}$ Rookie director $(0 / 1)_{t}$ & & $\begin{array}{c}-0.024^{* *} \\
(-2.46)\end{array}$ & & $\begin{array}{c}-0.010^{* * *} \\
(-3.71)\end{array}$ \\
\hline Women director $(\%)_{t}$ & $\begin{array}{l}0.037^{*} \\
(1.83)\end{array}$ & $\begin{array}{l}0.037^{*} \\
(1.84)\end{array}$ & $\begin{array}{l}0.008 \\
(1.53)\end{array}$ & $\begin{array}{l}0.008 \\
(1.57)\end{array}$ \\
\hline Busy director $(\%)_{t}$ & $\begin{array}{l}-0.013 \\
(-1.23)\end{array}$ & $\begin{array}{l}-0.016 \\
(-1.54)\end{array}$ & $\begin{array}{l}-0.003 \\
(-1.24)\end{array}$ & $\begin{array}{l}-0.004 \\
(-1.33)\end{array}$ \\
\hline Ln $(\text { Board size })_{t}$ & $\begin{array}{l}-0.022 \\
(-0.68)\end{array}$ & $\begin{array}{l}-0.025 \\
(-0.81)\end{array}$ & $\begin{array}{l}-0.006 \\
(-0.68)\end{array}$ & $\begin{array}{l}-0.005 \\
(-0.63)\end{array}$ \\
\hline Dualityt $_{t}$ & $\begin{array}{l}0.008 \\
(0.79)\end{array}$ & $\begin{array}{l}0.007 \\
(0.68)\end{array}$ & $\begin{array}{l}0.003 \\
(1.38)\end{array}$ & $\begin{array}{l}0.003 \\
(1.33)\end{array}$ \\
\hline Independent director $(\%)_{t}$ & $\begin{array}{l}0.018 \\
(0.25)\end{array}$ & $\begin{array}{l}0.013 \\
(0.18)\end{array}$ & $\begin{array}{l}-0.017 \\
(-0.92)\end{array}$ & $\begin{array}{l}-0.016 \\
(-0.86)\end{array}$ \\
\hline $\operatorname{Ln}(\text { Sales })_{t}$ & $\begin{array}{c}0.071^{* * *} \\
(5.87)\end{array}$ & $\begin{array}{c}0.072^{* * *} \\
(5.82)\end{array}$ & $\begin{array}{c}0.022^{* * *} \\
(10.98)\end{array}$ & $\begin{array}{c}0.022^{* * *} \\
(10.99)\end{array}$ \\
\hline Largest shareholder $(\%)_{t}$ & $\begin{array}{l}0.128^{*} \\
(1.91)\end{array}$ & $\begin{array}{l}0.122^{*} \\
(1.82)\end{array}$ & $\begin{array}{c}0.041^{* *} \\
(2.31)\end{array}$ & $\begin{array}{c}0.040^{* *} \\
(2.29)\end{array}$ \\
\hline $\operatorname{Ln}(\text { Firm age })_{t}$ & $\begin{array}{c}-0.010^{* * *} \\
(-4.59)\end{array}$ & $\begin{array}{c}-0.006^{* * *} \\
(-2.91)\end{array}$ & $\begin{array}{c}-0.003^{* * *} \\
(-5.82)\end{array}$ & $\begin{array}{c}-0.003^{* * *} \\
(-5.93)\end{array}$ \\
\hline Book leverage $_{t}$ & $\begin{array}{c}-0.454^{* * *} \\
(-10.70)\end{array}$ & $\begin{array}{c}-0.451^{* * *} \\
(-10.60)\end{array}$ & $\begin{array}{c}-0.139 * * * \\
(-15.83)\end{array}$ & $\begin{array}{c}-0.139 * * * \\
(-15.81)\end{array}$ \\
\hline$R \& D(\%)_{t}$ & $\begin{array}{l}-0.218 \\
(-1.30)\end{array}$ & $\begin{array}{l}-0.173 \\
(-1.06)\end{array}$ & $\begin{array}{c}-0.104^{*} \\
(-1.88)\end{array}$ & $\begin{array}{l}-0.103^{*} \\
(-1.87)\end{array}$ \\
\hline Year effects & Yes & Yes & Yes & Yes \\
\hline Firm effects & Yes & Yes & Yes & Yes \\
\hline$R^{2}$ & 0.112 & 0.118 & 0.182 & 0.182 \\
\hline Observations & 12,433 & 12,433 & 12,433 & 12,433 \\
\hline
\end{tabular}


Table 2.8: Robustness check: Rookie director appointments

In this table, we examine whether firm characteristics affect the appointments of rookie independent directors. The dependent variable Rookie appointment $(0 / 1)_{t}$ is a dummy variable that equal to 1 if a new appointed independent director is a rookie director and 0 otherwise. Table 2.12 provides all variable definitions. The regressions control for year, industry and firm fixed effects. In parentheses are t-statistics based on standard errors adjusted for heteroskedasticity and firm clustering. Superscripts $*{ }^{* *}$ and ${ }^{* * *}$ denote significance at the $10 \%, 5 \%$ and $1 \%$ levels, respectively.

\begin{tabular}{|c|c|c|}
\hline Explanatory variables & $\begin{array}{l}\text { Rookie } \\
\text { (1) }\end{array}$ & $\begin{array}{l}\text { ntment }(0 / 1)_{t} \\
(2)\end{array}$ \\
\hline$R O S_{t-1}$ & $\begin{array}{l}-0.007 \\
(-0.19)\end{array}$ & $\begin{array}{l}-0.018 \\
(-0.32)\end{array}$ \\
\hline$R O A_{t-1}$ & $\begin{array}{l}-0.149 \\
(-1.02)\end{array}$ & $\begin{array}{l}0.064 \\
(0.24)\end{array}$ \\
\hline $\operatorname{Ln}(\text { Board size })_{t-1}$ & $\begin{array}{c}0.034 \\
(0.96)\end{array}$ & $\begin{array}{l}-0.073 \\
(-0.75)\end{array}$ \\
\hline Duality $_{t-1}$ & $\begin{array}{l}-0.021 \\
(-1.32)\end{array}$ & $\begin{array}{l}-0.032 \\
(-0.86)\end{array}$ \\
\hline Independent director $(\%)_{t-1}$ & $\begin{array}{c}0.143 \\
(1.14)\end{array}$ & $\begin{array}{l}-0.012 \\
(-0.04)\end{array}$ \\
\hline State-owned $(0 / 1)_{t-1}$ & $\begin{array}{l}-0.003 \\
(-0.24)\end{array}$ & $\begin{array}{l}0.005 \\
(0.08)\end{array}$ \\
\hline Largest shareholder $(\%)_{t-1}$ & $\begin{array}{l}-0.071 \\
(-1.56)\end{array}$ & $\begin{array}{l}-0.014 \\
(-0.07)\end{array}$ \\
\hline $\operatorname{Ln}(\text { Sales })_{t-1}$ & $\begin{array}{l}-0.005 \\
(-1.06)\end{array}$ & $\begin{array}{l}-0.012 \\
(-0.61)\end{array}$ \\
\hline Book leverage $_{t-1}$ & $\begin{array}{l}-0.026 \\
(-0.79)\end{array}$ & $\begin{array}{l}0.046 \\
(0.60)\end{array}$ \\
\hline$R \mathscr{E} D(\%)_{t-1}$ & $\begin{array}{l}0.213 \\
(0.43)\end{array}$ & $\begin{array}{l}1.523 \\
(0.96)\end{array}$ \\
\hline$O R E C T A(\%)_{t-1}$ & $\begin{array}{l}-0.032 \\
(-0.22)\end{array}$ & $\begin{array}{l}-0.192 \\
(-0.70)\end{array}$ \\
\hline Year effects & Yes & Yes \\
\hline Industry effects & Yes & No \\
\hline Firm effects & $\mathrm{No}$ & Yes \\
\hline$R^{2}$ & 0.012 & 0.326 \\
\hline Observations & 7,284 & 7,284 \\
\hline
\end{tabular}


Table 2.9: Robustness check: The instrument variables 2SLS regressions

In this table, we rerun the regressions of rookie directors on firm performance with the instrument variable 2SLS method. We apply Retire director(\%)t-1 and First-year director(\%) $)_{-1}$ as our instrument variables. Retire director $(\%)_{t-1}$ is the mean value of the percent of independent directors of other firms headquartered in the same city leaving their boards due to the term limits at year t-1. First-year director $(\%)_{t-1}$ is the mean value of the percentage of first-year directors of other firms headquartered in the same city at year t-1. We use both Rookie director $(\%)_{t}$ and Rookie board $(0 / 1)_{t}$ to proxy the presence of rookie independent directors. Rookie director $(\%)_{t}$ is the ratio of rookie independent directors. Rookie board $(0 / 1)_{t}$ is a dummy variable that equals to 1 if the majority of independent directors are rookies. In Columns (1) and (2), we provide results of the first-stage of 2SLS regressions. In Columns (3) to (6) we provide results of the second-stage of $2 \mathrm{SLS}$ regressions. In Columns (3) and (4), the firm performance is measured by $R O S_{t}$. In Columns (5) and (6), the firm performance is measured by $R O A_{t}$. Table 2.12 provides all variable definitions. All regressions control for year and firm fixed effects. In parentheses are t-statistics based on standard errors adjusted for heteroskedasticity and firm clustering. Superscripts * ** and *** denote significance at the $10 \%, 5 \%$ and $1 \%$ levels, respectively.

\begin{tabular}{|c|c|c|c|c|c|c|}
\hline \multirow[b]{2}{*}{ Explanatory variables } & \multicolumn{2}{|c|}{ First stage of 2SLS regressions } & \multicolumn{4}{|c|}{ Second stage of 2SLS regressions } \\
\hline & $\begin{array}{c}\text { Rookie director }(\%)_{t} \\
(1)\end{array}$ & $\begin{array}{c}\text { Rookie board }(0 / 1)_{t} \\
(2)\end{array}$ & $\begin{array}{c}R O S(\text { Net I } \\
\text { (3) }\end{array}$ & $\begin{array}{c}\text { Income } / \text { Sales })_{t} \\
(4)\end{array}$ & $\begin{array}{c}R O A(\text { Net I } \\
(5)\end{array}$ & $\begin{array}{c}\text { ncome } / \text { Assets })_{t} \\
(6)\end{array}$ \\
\hline Retire director $(\%)_{t-1}$ & $\begin{array}{c}0.242^{* * *} \\
(9.48)\end{array}$ & $\begin{array}{c}0.207^{* * *} \\
(5.65)\end{array}$ & & & & \\
\hline First-year director $(\%)_{t-1}$ & $\begin{array}{c}0.340^{* * *} \\
(18.41)\end{array}$ & $\begin{array}{c}0.367^{* * *} \\
(14.27)\end{array}$ & & & & \\
\hline Rookie director $(\%)_{t}$ & & & $\begin{array}{c}0.070^{* *} \\
(2.19)\end{array}$ & & $\begin{array}{c}0.021^{* *} \\
(2.06)\end{array}$ & \\
\hline Rookie board $(0 / 1)_{t}$ & & & & $\begin{array}{c}0.067^{* *} \\
(2.13)\end{array}$ & & $\begin{array}{c}0.020^{* *} \\
(2.02)\end{array}$ \\
\hline Women director $(\%)_{t}$ & $\begin{array}{l}0.062^{*} \\
(1.74)\end{array}$ & $\begin{array}{c}0.077^{*} \\
(1.91)\end{array}$ & $\begin{array}{l}0.023 \\
(0.99)\end{array}$ & $\begin{array}{l}0.022 \\
(0.96)\end{array}$ & $\begin{array}{l}0.007 \\
(1.30)\end{array}$ & $\begin{array}{l}0.007 \\
(1.26)\end{array}$ \\
\hline Busy director $(\%)_{t}$ & $\begin{array}{c}-0.088^{* * *} \\
(-5.26)\end{array}$ & $\begin{array}{c}-0.040^{* *} \\
(-2.04)\end{array}$ & $\begin{array}{l}0.006 \\
(0.44)\end{array}$ & $\begin{array}{l}0.002 \\
(0.17)\end{array}$ & $\begin{array}{l}0.004 \\
(1.20)\end{array}$ & $\begin{array}{l}0.003 \\
(0.93)\end{array}$ \\
\hline Ln(Board size $)_{t}$ & $\begin{array}{c}0.081^{* *} \\
(2.00)\end{array}$ & $\begin{array}{l}0.062 \\
(1.28)\end{array}$ & $\begin{array}{l}-0.012 \\
(-0.33)\end{array}$ & $\begin{array}{l}-0.011 \\
(-0.29)\end{array}$ & $\begin{array}{l}0.007 \\
(0.75)\end{array}$ & $\begin{array}{l}0.008 \\
(0.79)\end{array}$ \\
\hline Duality $_{t}$ & $\begin{array}{l}0.016 \\
(1.13)\end{array}$ & $\begin{array}{c}0.033^{*} \\
(1.87)\end{array}$ & $\begin{array}{l}-0.006 \\
(-0.48)\end{array}$ & $\begin{array}{l}-0.007 \\
(-0.56)\end{array}$ & $\begin{array}{l}-0.000 \\
(-0.10)\end{array}$ & $\begin{array}{l}-0.001 \\
(-0.20)\end{array}$ \\
\hline Independent director $(\%)_{t}$ & $\begin{array}{l}0.159 \\
(1.41)\end{array}$ & $\begin{array}{l}0.037 \\
(0.28)\end{array}$ & $\begin{array}{l}0.005 \\
(0.06)\end{array}$ & $\begin{array}{l}0.013 \\
(0.16)\end{array}$ & $\begin{array}{l}-0.009 \\
(-0.39)\end{array}$ & $\begin{array}{l}-0.006 \\
(-0.27)\end{array}$ \\
\hline Ln(Sales) $t$ & $\begin{array}{c}-0.012^{*} \\
(-1.69)\end{array}$ & $\begin{array}{l}-0.013 \\
(-1.58)\end{array}$ & $\begin{array}{l}-0.006 \\
(-0.57)\end{array}$ & $\begin{array}{l}-0.006 \\
(-0.57)\end{array}$ & $\begin{array}{l}-0.001 \\
(-0.58)\end{array}$ & $\begin{array}{l}-0.001 \\
(-0.58)\end{array}$ \\
\hline State-owned $(0 / 1)_{t}$ & $\begin{array}{l}-0.024 \\
(-1.08)\end{array}$ & $\begin{array}{l}-0.014 \\
(-0.48)\end{array}$ & $\begin{array}{l}-0.038 \\
(-1.13)\end{array}$ & $\begin{array}{l}-0.039 \\
(-1.16)\end{array}$ & $\begin{array}{c}-0.023^{* * *} \\
(-2.94)\end{array}$ & $\begin{array}{c}-0.023^{* * *} \\
(-3.01)\end{array}$ \\
\hline Largest shareholder $(\%)_{t}$ & $\begin{array}{l}-0.086 \\
(-1.13)\end{array}$ & $\begin{array}{l}-0.009 \\
(-0.10)\end{array}$ & $\begin{array}{c}0.154^{* *} \\
(2.25)\end{array}$ & $\begin{array}{c}0.148^{* *} \\
(2.20)\end{array}$ & $\begin{array}{l}0.025 \\
(1.58)\end{array}$ & $\begin{array}{l}0.024 \\
(1.48)\end{array}$ \\
\hline $\operatorname{Ln}\left(\right.$ Firm age $_{t}$ & $\begin{array}{c}-0.025^{* * *} \\
(-10.01)\end{array}$ & $\begin{array}{c}-0.034^{* * *} \\
(-10.52)\end{array}$ & $\begin{array}{c}-0.018^{* * *} \\
(-3.54)\end{array}$ & $\begin{array}{c}-0.016^{* * *} \\
(-3.05)\end{array}$ & $\begin{array}{c}-0.005^{* * *} \\
(-4.14)\end{array}$ & $\begin{array}{c}-0.004^{* * *} \\
(-3.64)\end{array}$ \\
\hline Book leverage $_{t}$ & $\begin{array}{l}0.060^{*} \\
(1.92)\end{array}$ & $\begin{array}{c}0.076^{*} \\
(1.82)\end{array}$ & $\begin{array}{l}0.060 \\
(1.47)\end{array}$ & $\begin{array}{l}0.059 \\
(1.44)\end{array}$ & $\begin{array}{l}0.015 \\
(1.59)\end{array}$ & $\begin{array}{l}0.015 \\
(1.56)\end{array}$ \\
\hline$R \& D(\%)_{t}$ & $\begin{array}{l}-0.748 \\
(-1.40)\end{array}$ & $\begin{array}{c}-1.237^{* *} \\
(-2.02)\end{array}$ & $\begin{array}{l}-0.233 \\
(-1.04)\end{array}$ & $\begin{array}{l}-0.203 \\
(-0.90)\end{array}$ & $\begin{array}{l}-0.054 \\
(-0.85)\end{array}$ & $\begin{array}{l}-0.045 \\
(-0.69)\end{array}$ \\
\hline First-stage $\mathrm{F}$ test statistics & 45.29 & 30.03 & & & & \\
\hline Over-identification test p-value & & & 0.86 & 0.71 & 0.88 & 0.96 \\
\hline Year effects & Yes & Yes & Yes & Yes & Yes & Yes \\
\hline Firm effects & Yes & Yes & Yes & Yes & Yes & Yes \\
\hline$R^{2}$ & 0.102 & 0.086 & 0.006 & 0.006 & 0.017 & 0.011 \\
\hline Observations & 10,234 & 10,234 & 10,194 & 10,194 & 10,194 & 10,194 \\
\hline
\end{tabular}


Table 2.10: Robustness check: Alternative measure of ROS and ROA

In this table, we rerun the regressions of rookie directors on firm performance with alternative measure of $R O S_{t}$ and $R O A_{t}$. We replace net income by EBITDA to calculate $R O S_{t}$ and $R O A_{t}$. We use both Rookie director $(\%)_{t}$ and Rookie board $(0 / 1)_{t}$ to proxy the presence of rookie independent directors. Rookie director $(\%)_{t}$ is the ratio of rookie independent directors. Rookie $\operatorname{board}(0 / 1)_{t}$ is a dummy variable that equals to 1 if the majority of independent directors are rookies. In Columns (1) and (2), the firm performance is measured by $R O S_{t}$. In Columns (3) and (4), the firm performance is measured by $R O A_{t}$. Table 2.12 provides all variable definitions. All regressions control for year and firm fixed effects. In parentheses are t-statistics based on standard errors adjusted for heteroskedasticity and firm clustering. Superscripts *, ** and $* * *$ denote significance at the $10 \%, 5 \%$ and $1 \%$ levels, respectively.

\begin{tabular}{|c|c|c|c|c|}
\hline \multirow[b]{2}{*}{ Explanatory variables } & \multicolumn{2}{|c|}{$R O S(E B I T D A / \text { Sales })_{t}$} & \multicolumn{2}{|c|}{$R O A(E B I T D A / A s s e t s)_{t}$} \\
\hline & $(1)$ & $(2)$ & (3) & $(4)$ \\
\hline Rookie director $(\%)_{t}$ & $\begin{array}{c}0.015^{* * *} \\
(2.66)\end{array}$ & & $\begin{array}{c}0.004^{* *} \\
(2.32)\end{array}$ & \\
\hline Rookie board $(0 / 1)_{t}$ & & $\begin{array}{c}0.013^{* * *} \\
(3.01)\end{array}$ & & $\begin{array}{c}0.003^{* *} \\
(2.09)\end{array}$ \\
\hline Women director $(\%)_{t}$ & $\begin{array}{l}0.017 \\
(1.04)\end{array}$ & $\begin{array}{l}0.017 \\
(1.04)\end{array}$ & $\begin{array}{l}0.007 \\
(1.28)\end{array}$ & $\begin{array}{l}0.007 \\
(1.29)\end{array}$ \\
\hline Busy director $(\%)_{t}$ & $\begin{array}{l}-0.005 \\
(-0.50)\end{array}$ & $\begin{array}{l}-0.006 \\
(-0.58)\end{array}$ & $\begin{array}{l}-0.004 \\
(-1.36)\end{array}$ & $\begin{array}{l}-0.004 \\
(-1.44)\end{array}$ \\
\hline $\operatorname{Ln}(\text { Board size })_{t}$ & $\begin{array}{l}-0.011 \\
(-0.39)\end{array}$ & $\begin{array}{l}-0.010 \\
(-0.37)\end{array}$ & $\begin{array}{l}-0.008 \\
(-0.96)\end{array}$ & $\begin{array}{l}-0.008 \\
(-0.94)\end{array}$ \\
\hline Duality $_{t}$ & $\begin{array}{l}0.004 \\
(0.62)\end{array}$ & $\begin{array}{l}0.004 \\
(0.60)\end{array}$ & $\begin{array}{l}0.002 \\
(0.96)\end{array}$ & $\begin{array}{l}0.002 \\
(0.95)\end{array}$ \\
\hline Independent director $(\%)_{t}$ & $\begin{array}{l}0.002 \\
(0.03)\end{array}$ & $\begin{array}{l}0.005 \\
(0.07)\end{array}$ & $\begin{array}{l}-0.006 \\
(-0.32)\end{array}$ & $\begin{array}{l}-0.005 \\
(-0.28)\end{array}$ \\
\hline State-owned $(0 / 1)_{t}$ & $\begin{array}{c}-0.046^{* *} \\
(-2.07)\end{array}$ & $\begin{array}{c}-0.047^{* *} \\
(-2.09)\end{array}$ & $\begin{array}{c}-0.024^{* * *} \\
(-3.94)\end{array}$ & $\begin{array}{c}-0.024^{* * *} \\
(-3.97)\end{array}$ \\
\hline Largest shareholder $(\%)_{t}$ & $\begin{array}{c}0.161^{* * *} \\
(2.65)\end{array}$ & $\begin{array}{c}0.160^{* * *} \\
(2.64)\end{array}$ & $\begin{array}{l}0.028 \\
(1.55)\end{array}$ & $\begin{array}{l}0.028 \\
(1.55)\end{array}$ \\
\hline $\operatorname{Ln}(\text { Sales })_{t}$ & $\begin{array}{l}-0.003 \\
(-0.35)\end{array}$ & $\begin{array}{l}-0.003 \\
(-0.34)\end{array}$ & $\begin{array}{c}0.023^{* * *} \\
(10.84)\end{array}$ & $\begin{array}{c}0.023^{* * *} \\
(10.85)\end{array}$ \\
\hline Ln(Firm age $)_{t}$ & $\begin{array}{c}0.002 \\
(1.20)\end{array}$ & $\begin{array}{l}0.002 \\
(1.22)\end{array}$ & $\begin{array}{c}-0.004^{* * *} \\
(-9.16)\end{array}$ & $\begin{array}{c}-0.004^{* * *} \\
(-9.29)\end{array}$ \\
\hline Book leverage $_{t}$ & $\begin{array}{c}-0.197^{* * *} \\
(-6.22)\end{array}$ & $\begin{array}{c}-0.197^{* * *} \\
(-6.21)\end{array}$ & $\begin{array}{c}-0.083^{* * *} \\
(-9.57)\end{array}$ & $\begin{array}{c}-0.083^{* * *} \\
(-9.56)\end{array}$ \\
\hline$R \& D(\%)_{t}$ & $\begin{array}{l}-0.195 \\
(-1.35)\end{array}$ & $\begin{array}{l}-0.194 \\
(-1.35)\end{array}$ & $\begin{array}{c}-0.157^{* * * *} \\
(-3.10)\end{array}$ & $\begin{array}{c}-0.158^{* * *} \\
(-3.12)\end{array}$ \\
\hline Year effects & Yes & Yes & Yes & Yes \\
\hline Firm effects & Yes & Yes & Yes & Yes \\
\hline$R^{2}$ & 0.037 & 0.037 & 0.120 & 0.120 \\
\hline Observations & 12,433 & 12,433 & 12,433 & 12,433 \\
\hline
\end{tabular}


Table 2.11: Robustness check: Last-year director and board meeting attendance

In this table, we rerun the regressions of rookie directors on board meeting attendance by including a variable Last-year director $(0 / 1)_{t}$, a dummy variable equals to 1 if an independent director is near the end of term (normally year 6 or year 7 ) and 0 otherwise. In Columns (1) to (4), the dependent variable is Meeting absence $(0 / 1)_{t}$, a dummy variable that equals to 1 if an independent director absents any board meetings and 0 otherwise. In Columns (5) to (8), the dependent variable is Meeting absence $(\%)_{t}$, the ratio of board meeting absences. The measure of rookie independent director is Rookie director $(0 / 1)_{t}$, a dummy variable that equal to 1 if an independent director has less than three years' board experience and 0 otherwise. Table 2.12 provides all variable definitions. The regressions control for year, industry, firm, director and firm*year fixed effects. In parentheses are t-statistics based on standard errors adjusted for heteroskedasticity and director clustering. Superscripts ${ }^{*}, * *$ and ${ }^{* * *}$ denote significance at the $10 \%, 5 \%$ and $1 \%$ levels, respectively.

\begin{tabular}{|c|c|c|c|c|c|c|c|c|}
\hline \multirow[b]{2}{*}{ Explanatory variables } & \multicolumn{4}{|c|}{ Meeting absence $(0 / 1)_{t}$} & \multicolumn{4}{|c|}{ Meeting absence $(\%)_{t}$} \\
\hline & (1) & $(2)$ & (3) & (4) & (5) & (6) & (7) & (8) \\
\hline Rookie director $(0 / 1)_{t}$ & $\begin{array}{c}-0.011^{* *} \\
(-2.21)\end{array}$ & $\begin{array}{c}-0.014^{* * *} \\
(-2.84)\end{array}$ & $\begin{array}{c}-0.015^{* *} \\
(-2.26)\end{array}$ & $\begin{array}{c}-0.014^{* *} \\
(-2.37)\end{array}$ & $\begin{array}{c}-0.004^{* * *} \\
(-3.07)\end{array}$ & $\begin{array}{c}-0.005^{* * *} \\
(-3.83)\end{array}$ & $\begin{array}{c}-0.004^{* *} \\
(-2.35)\end{array}$ & $\begin{array}{c}-0.005^{* * *} \\
(-2.79)\end{array}$ \\
\hline Last-year director $(0 / 1)_{t}$ & $\begin{array}{c}0.059^{* * *} \\
(8.92)\end{array}$ & $\begin{array}{c}0.063^{* * *} \\
(9.73)\end{array}$ & $\begin{array}{c}0.042^{* * *} \\
(5.72)\end{array}$ & $\begin{array}{c}0.085^{* * *} \\
(10.25)\end{array}$ & $\begin{array}{c}0.023^{* * *} \\
(10.03)\end{array}$ & $\begin{array}{c}0.024^{* * *} \\
(10.24)\end{array}$ & $\begin{array}{c}0.018^{* * *} \\
(7.23)\end{array}$ & $\begin{array}{c}0.032^{* * *} \\
(8.64)\end{array}$ \\
\hline $\operatorname{Woman}(0 / 1)$ & $\begin{array}{l}-0.005 \\
(-1.01)\end{array}$ & $\begin{array}{l}-0.007 \\
(-1.24)\end{array}$ & & $\begin{array}{c}-0.012^{* *} \\
(-2.18)\end{array}$ & $\begin{array}{l}-0.001 \\
(-0.57)\end{array}$ & $\begin{array}{l}-0.001 \\
(-0.56)\end{array}$ & & $\begin{array}{l}-0.001 \\
(-0.67)\end{array}$ \\
\hline $\operatorname{Age}(\text { Ten years })_{t}$ & $\begin{array}{c}-0.006 * * * \\
(-2.99)\end{array}$ & $\begin{array}{c}-0.007^{* * *} \\
(-2.96)\end{array}$ & $\begin{array}{l}0.014 \\
(0.13)\end{array}$ & $\begin{array}{c}-0.007^{* * *} \\
(-3.06)\end{array}$ & $\begin{array}{c}-0.002^{* *} \\
(-2.12)\end{array}$ & $\begin{array}{c}-0.001^{* *} \\
(-1.99)\end{array}$ & $\begin{array}{l}0.022 \\
(0.63)\end{array}$ & $\begin{array}{c}-0.002^{*} \\
(-1.82)\end{array}$ \\
\hline Tenure in firm(Years) $)_{t}$ & $\begin{array}{c}0.003^{* *} \\
(2.31)\end{array}$ & $\begin{array}{l}0.001 \\
(0.67)\end{array}$ & $\begin{array}{c}0.006^{* * *} \\
(4.01)\end{array}$ & $\begin{array}{l}0.000 \\
(0.17)\end{array}$ & $\begin{array}{l}0.001 \\
(1.53)\end{array}$ & $\begin{array}{l}0.000 \\
(0.93)\end{array}$ & $\begin{array}{c}0.001^{* *} \\
(2.27)\end{array}$ & $\begin{array}{l}0.001 \\
(0.98)\end{array}$ \\
\hline Busy director $(0 / 1)_{t}$ & $\begin{array}{l}0.006 \\
(1.18)\end{array}$ & $\begin{array}{l}0.005 \\
(1.01)\end{array}$ & $\begin{array}{l}-0.001 \\
(-0.15)\end{array}$ & $\begin{array}{l}0.004 \\
(0.61)\end{array}$ & $\begin{array}{l}-0.001 \\
(-1.10)\end{array}$ & $\begin{array}{l}-0.001 \\
(-0.74)\end{array}$ & $\begin{array}{l}-0.003 \\
(-1.52)\end{array}$ & $\begin{array}{l}-0.001 \\
(-0.80)\end{array}$ \\
\hline 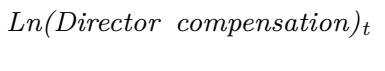 & $\begin{array}{c}0.003^{* * *} \\
(4.02)\end{array}$ & $\begin{array}{l}0.001 \\
(1.33)\end{array}$ & $\begin{array}{c}0.003^{* * *} \\
(3.85)\end{array}$ & $\begin{array}{l}0.000 \\
(0.44)\end{array}$ & $\begin{array}{l}-0.000 \\
(-1.57)\end{array}$ & $\begin{array}{c}-0.001^{* *} \\
(-2.14)\end{array}$ & $\begin{array}{l}-0.000 \\
(-0.77)\end{array}$ & $\begin{array}{l}-0.001 \\
(-1.15)\end{array}$ \\
\hline Political backgrounds $(0 / 1)_{t}$ & $\begin{array}{l}0.005 \\
(1.18)\end{array}$ & $\begin{array}{l}0.003 \\
(0.82)\end{array}$ & $\begin{array}{c}-0.224^{* * *} \\
(-3.68)\end{array}$ & $\begin{array}{l}0.004 \\
(0.84)\end{array}$ & $\begin{array}{l}0.001 \\
(0.82)\end{array}$ & $\begin{array}{l}0.001 \\
(0.42)\end{array}$ & $\begin{array}{l}-0.021 \\
(-0.77)\end{array}$ & $\begin{array}{l}0.001 \\
(0.41)\end{array}$ \\
\hline Meeting frequency $y_{t}$ & $\begin{array}{c}0.009 * * * \\
(16.79)\end{array}$ & $\begin{array}{c}0.012^{* * *} \\
(19.20)\end{array}$ & $\begin{array}{c}0.010 * * * \\
(15.69)\end{array}$ & $\begin{array}{c}0.013^{* * *} \\
(12.27)\end{array}$ & $\begin{array}{c}-0.001^{* * * *} \\
(-6.69)\end{array}$ & $\begin{array}{c}-0.001^{* * *} \\
(-4.85)\end{array}$ & $\begin{array}{c}-0.001 * * * \\
(-6.01)\end{array}$ & $\begin{array}{c}-0.002^{* * * *} \\
(-3.11)\end{array}$ \\
\hline Ln(Board size $)_{t}$ & $\begin{array}{c}0.166^{* * *} \\
(15.57)\end{array}$ & $\begin{array}{l}0.005 \\
(0.21)\end{array}$ & $\begin{array}{c}0.087^{* * *} \\
(4.97)\end{array}$ & & $\begin{array}{c}0.039 * * * \\
(8.18)\end{array}$ & $\begin{array}{l}0.010 \\
(1.39)\end{array}$ & $\begin{array}{c}0.021^{* * *} \\
(4.26)\end{array}$ & \\
\hline Duality $_{t}$ & $\begin{array}{l}-0.002 \\
(-0.44)\end{array}$ & $\begin{array}{l}0.001 \\
(0.10)\end{array}$ & $\begin{array}{l}-0.003 \\
(-0.40)\end{array}$ & & $\begin{array}{l}0.002 \\
(0.84)\end{array}$ & $\begin{array}{l}0.003 \\
(1.40)\end{array}$ & $\begin{array}{l}0.001 \\
(0.68)\end{array}$ & \\
\hline Independent director $(\%)_{t}$ & $\begin{array}{c}0.148^{* * *} \\
(4.14)\end{array}$ & $\begin{array}{l}-0.101 \\
(-1.51)\end{array}$ & $\begin{array}{l}0.084 \\
(1.53)\end{array}$ & & $\begin{array}{c}0.051^{* * *} \\
(3.19)\end{array}$ & $\begin{array}{l}-0.012 \\
(-0.55)\end{array}$ & $\begin{array}{c}0.039^{* *} \\
(2.46)\end{array}$ & \\
\hline State-owned $(0 / 1)_{t}$ & $\begin{array}{c}0.037^{* * *} \\
(8.65)\end{array}$ & $\begin{array}{l}0.022 \\
(1.37)\end{array}$ & $\begin{array}{c}0.038^{* * *} \\
(4.91)\end{array}$ & & $\begin{array}{c}0.008^{* * *} \\
(4.51)\end{array}$ & $\begin{array}{l}0.006 \\
(0.88)\end{array}$ & $\begin{array}{c}0.008^{* * *} \\
(3.68)\end{array}$ & \\
\hline Largest shareholder $(\%)_{t}$ & $\begin{array}{l}-0.018 \\
(-1.44)\end{array}$ & $\begin{array}{l}-0.047 \\
(-1.18)\end{array}$ & $\begin{array}{c}-0.052^{* *} \\
(-2.37)\end{array}$ & & $\begin{array}{l}-0.007 \\
(-1.23)\end{array}$ & $\begin{array}{l}-0.006 \\
(-0.54)\end{array}$ & $\begin{array}{c}-0.014^{* *} \\
(-2.35)\end{array}$ & \\
\hline Ln $(\text { Sales })_{t}$ & $\begin{array}{l}-0.000 \\
(-0.19)\end{array}$ & $\begin{array}{l}-0.004 \\
(-0.96)\end{array}$ & $\begin{array}{c}-0.005^{*} \\
(-1.82)\end{array}$ & & $\begin{array}{l}0.000 \\
(0.41)\end{array}$ & $\begin{array}{l}-0.001 \\
(-0.43)\end{array}$ & $\begin{array}{l}-0.001 \\
(-0.71)\end{array}$ & \\
\hline Book leverage $_{t}$ & $\begin{array}{c}0.040^{* * *} \\
(4.00)\end{array}$ & $\begin{array}{l}-0.000 \\
(-0.01)\end{array}$ & $\begin{array}{c}0.062^{* * *} \\
(4.00)\end{array}$ & & $\begin{array}{c}0.011^{* *} \\
(2.43)\end{array}$ & $\begin{array}{l}0.002 \\
(0.28)\end{array}$ & $\begin{array}{c}0.012^{* * *} \\
(2.61)\end{array}$ & \\
\hline$R O A_{t}$ & $\begin{array}{l}0.004 \\
(0.11)\end{array}$ & $\begin{array}{l}0.027 \\
(0.57)\end{array}$ & $\begin{array}{c}0.093^{* *} \\
(2.02)\end{array}$ & & $\begin{array}{l}-0.002 \\
(-0.16)\end{array}$ & $\begin{array}{l}0.003 \\
(0.22)\end{array}$ & $\begin{array}{l}0.017 \\
(1.21)\end{array}$ & \\
\hline Year & Yes & Yes & Yes & No & Yes & Yes & Yes & No \\
\hline Industry effects & Yes & No & Yes & No & Yes & No & Yes & No \\
\hline Firm effects & No & Yes & No & No & No & Yes & No & No \\
\hline Director effects & No & No & Yes & No & No & No & Yes & No \\
\hline Firm*year effects & No & No & No & Yes & No & No & No & Yes \\
\hline$R^{2}$ & 0.032 & 0.188 & 0.345 & 0.415 & 0.028 & 0.156 & 0.367 & 0.359 \\
\hline Observations & 42,608 & 42,608 & 42,608 & 42,608 & 42,608 & 42,608 & 42,608 & 42,608 \\
\hline
\end{tabular}




\subsection{Appendix}

Table 2.12: Variable definitions

Variable Description

\section{Dependent variables}

Meeting absence $(0 / 1)_{t}$

Meeting absence $(\%)_{t}$ and 0 otherwise.

$R O A_{t}$

The ratio of board meeting absences, which equals to the number of board meeting absented scaled by the total number of board meeting in the year $t$.

$\stackrel{\infty}{\infty} R_{t}$

$\operatorname{ORECTA}(\%)_{t}$

Turnover $(0 / 1)_{t+1}$

Rookie appointment $(0 / 1)_{t}$

\section{Variables of interest}

Rookie director $(0 / 1)_{t}$
The net income scaled by the book value of total assets in the year $t$.

The net income scaled by the sales in the year $t$.

The other receivables scaled by total assets in the year $t$.

The dummy variable measures whether an independent director loses one of his or her directorships in the year $t+1$. This dummy variable equals to 1 for an observation in year $t+1$ if an independent director does not appear in the annual report in year $t+2$ and 0 otherwise.

The dummy variable equals to 1 if the new appointed independent director is a rookie director in the year $t$ and 0 otherwise.

The dummy variable equals to 1 if an independent director has less than three years of directorship experience in the year $t$ and 0 otherwise. 
Table 2.12: Variable definitions

Variable

Rookie director $(\%)_{t}$

Rookie board $(0 / 1)_{t}$

Retire director $(\%)_{t-1}$

First-year director $(\%)_{t-1}$

\section{$\stackrel{\infty}{ }$ Control variables}

$\operatorname{Woman}(0 / 1)$

Women director $(\%)_{t}$

Busy director $(0 / 1)_{t}$

Busy director (\%)t

Political backgrounds(0/1)t

Age(Ten years) ${ }_{t}$

Tenure in firm(Years)

Ln(Director compensation) ${ }_{t}$

\section{Description}

The number of rookie independent directors scaled by the number of independent directors in the year t.

The dummy variable equals to 1 if more than $50 \%$ of independent directors are rookie directors in the year $t$ and 0 otherwise.

The mean value of the percentage of independent directors of other firms headquartered in the same city leaving their boards due to the term limits in the year $t-1$.

The mean value of the percentage of first-year directors of other firms headquartered in the same city in the year $t-1$.

The dummy variable equals to 1 if an independent director is female and 0 otherwise.

The number of female directors scaled by the number of independent directors in the year $t$.

The dummy variable equals to 1 if an independent director has more than two directorships in the year $t$ and 0 otherwise.

The number of busy directors scaled by the number of independent directors in the year $t$.

The dummy variable equals to 1 if an independent director has political background in the year $t$ and 0 otherwise.

The age of an independent director scaled by 10 in the year $t$.

The number of years that an independent director has served in a firm in the year $t$.

The logarithm of annual independent director compensation plus one in the year $t$ 
Table 2.12: Variable definitions

Variable

Description

Ln(Number of directorships)t The logarithm of the number of directorships that an independent director holds in the year $t$.

Ln(Meeting frequency) $)_{t}$

The logarithm of the number of board meeting that an independent director is required to attend in the year $t$.

Ln(Board size $)_{t}$

The logarithm of the number of directors on the board in the year $t$.

Independent director $(\%)_{t}$

The ratio of independent directors on the board in the year $t$.

Duality $_{t}$

The dummy variable equals to 1 if the $\mathrm{CEO}$ and chairman is the same person in the year $t$ and 0 otherwise.

State-owned $(0 / 1)_{t} \quad$ The dummy variable equals to 1 if the firm is state-owned in the year $t$ and 0 otherwise.

$\stackrel{\infty}{c}$

Largest shareholder $(\%)_{t}$

The percentage of share is holding by the largest shareholders in the year $t$.

Total assets(Billions $C N Y)_{t}$

The book values of total assets in the year $t$.

Ln(Sales) $)_{t}$

The logarithm of sales in the year $t$.

Ln(Firm age $)_{t}$

The logarithm of firm age in the year $t$.

Book leverage

The book value of total debts scaled by book value of total assets in the year $t$.

$R \& D(\%)_{t}$

The development expenses scaled by the sales in the year $t$. 
Chapter 3

Board of director compensation in

China: It pays to be connected 


\subsection{Introduction}

Boards of different firms are connected through common board members. These board connections form director networks. Director networks facilitate information transfer among boards, leading well-connected directors to be more informed. Through superior information, a well-connected director may serve as a better adviser or a more efficient monitor. ${ }^{1}$ In addition, board connections of directors may reflect the managerial talent and past success that signal director quality (Fama and Jensen, 1983, Renneboog and Zhao, 2011, Intintoli, Kahle, and Zhao, 2018). Indeed, recent literature reflects these advantages by illustrating how highly connected individuals fare better in their careers. For example, Ferris, David, and Yun (2016) find that U.S. firms increase the compensation of directors with network connections. Renneboog and Zhao (2018) find that in the U.K. director networks provide directors with access to labor market information. As a result, well-connected directors are more likely to leave their current position for another firm.

Although director networks have received academic attention, most studies focus on directors from western boards. There is limited research on the role of board networks in the development of a director's career in China, where the ownership structure and governance issues differ from those in the U.S. and U.K. (Jiang and Kim, 2015). This study examines how director networks affect director career outcomes in China. Our study addresses the following questions. How do board networks influence director compensation? How do director networks influence a director's job mobility? Are directors rewarded with additional future directorships for their network connections? We are interested in how the answers to these questions differ between Chinese and western boards.

We answer these questions by studying the unique structure of board memberships in China. Because board networks may have different effects on career outcomes for each type of director, we separate the board members into independent directors, executive directors and non-independent non-executive directors. ${ }^{2}$ In addition, we categorize nonindependent directors into related directors and non-related directors basing on whether a non-independent director holds a position in the controlling firms. ${ }^{3}$ This classification isolates the effects of board network on career outcomes between related directors and nonrelated directors.

To examine the influence of board networks on director career outcomes, our study requires measures of director connections. We borrow these measures from graph theory. ${ }^{4}$

\footnotetext{
${ }^{1}$ For example, Cai and Sevilir (2012) find that in the U.S. well-connected directors benefit firms in M\&A transactions by providing private information about target firms. This information advantage allows acquiring firms to pay lower takeover premiums. Intintoli, Kahle, and Zhao (2018) find that the presence of well-connected independent directors improves financial reporting quality in U.S. firms.

${ }^{2}$ See Section 3.3.1 for classification.

${ }^{3}$ See Table 3.14 for the definition of related directors.

${ }^{4}$ Graph theory is a mathematical discipline. It has been widely used to model network in economics.
} 
In graph theory, centrality measures the relative importance of each agent in a network. Since centrality measures are highly correlated, in our main results, we adopt eigenvector centrality to measure the influence of a director in a board network. Eigenvector centrality measures both the number of agent connections and the number of connections of an agent's connections. Following Koka and Prescott (2008), we name eigenvector centrality network prominence.

We first explore the influence of director networks on directorship level compensation. Following Chen and Keefe (2018), our compensation measures include both the propensity of a director to be paid in a firm and the level of compensation that a director receives from a firm in a given year. We find that independent directors with higher network prominence receive higher compensation. Our findings regarding independent director compensation are consistent with the literature suggesting that board connections increase director value, which is positively priced in board compensation (Intintoli, Kahle, and Zhao, 2018).

We then examine whether director turnover increases with director network prominence. We find that well-connected board members experience more turnover. Our results are consistent with the literature arguing that director networks may provide directors with information about better external directorship opportunities, leading to an increase in turnover (Renneboog and Zhao, 2018). In contrast, we find related directors experience less turnover than non-related directors. ${ }^{5}$ This effect is stronger for related directors' board connections. The above results are consistent with the literature suggesting that in the U.S. and U.K. board connections grant non-independent directors managerial power which shields them from dismissal, leading more connected directors to have less turnover (Renneboog and Zhao, 2011, Intintoli, Kahle, and Zhao, 2018). Overall, our study suggests a mixed effect of board networks on director job mobility. That is, board connections increase turnover for non-related directors to facilitate their access to better external opportunities, whereas board connections reduce turnover to protect related directors from dismissal.

We also investigate whether network prominence leads to directors obtaining future directorships. We find that well-connected directors receive more future directorships than less connected directors. This finding is consistent with the literature that well-connected directors are rewarded with more additional directorships due to either their better advising and monitoring functions or superior information in the labor market (Larcker and Tayan, 2010, Cai and Sevilir, 2012, Renneboog and Zhao, 2014, Larcker, So, and Wang, 2013, Fama and Jensen, 1983, Ferris, Jagannathan, and Pritchard, 2003, Renneboog and Zhao, 2018). In addition, we find that related non-independent non-executive directors (holding positions in controlling firms) gain more future directorships than other non-independent non-executive directors (not holding positions in controlling firms). This effect is stronger

\footnotetext{
${ }^{5}$ We define related directors as those who hold positions in controlling firms. See Table 3.14 for all variable definitions.
} 
when these related directors have more board connections. Overall, we find that network prominence rewards directors with more future directorships. However, for non-independent non-executive directors, network prominence only leads to more future board seats for directors who hold positions in controlling firms (related directors).

Moreover, network prominence may indirectly increase total director compensation. For example, we find that well-connected independent directors receive higher total compensation through navigating from low paid directorships to high paid directorships (director turnover channel). Furthermore, we find that well-connected independent directors receive higher total compensation from holding more board seats.

The remainder of the paper is organized as follows. Section 2 discusses relevant literature and develops the hypothesis. Section 3 presents sample and variables construction. Section 4 reports the empirical testing method and the main empirical results. Section 5 conducts the robustness tests. The final section concludes the paper.

\subsection{Literature and hypothesis development}

Prior literature suggests that well-connected directors improve firm decision making through access to superior information (Larcker and Tayan, 2010, Renneboog and Zhao, 2011). For example, Cai and Sevilir (2012) show how director connections benefit acquiring firms in M\&A transactions in the U.S.. They find that board connections to target firms provide the acquirers with private information about target firms. This information advantage deters competition from less-informed outside bidders (winner's curse) and allows acquirers to have greater bargaining power in merger negotiation. As a result, well-connected acquirers pay lower takeover premiums. Moreover, Renneboog and Zhao (2014) demonstrate that director networks facilitate takeover activity among firms in the U.K.. They observe that better networked firms are more active bidders in the takeover market and that board connections through interlocking directorships lead to higher takeover transaction success rates and shorter negotiation periods. Besides, superior information gained through board connections may improve monitoring. For instance, Intintoli, Kahle, and Zhao (2018) find evidence that board connections of independent, non co-opted audit committee members improve financial reporting quality in U.S. firms. Consistent with potential benefits from board connections, Larcker, So, and Wang (2013) demonstrate that in the U.S. well-connected firms are more profitable and have higher abnormal returns.

If board connections benefit firms' decision making and corporate governance, firms will demand well-connected directors and pay for these board connections. Furthermore, the relative position of a director in the network may reflect managerial talent and past success, which are signals of director quality (Fama and Jensen, 1983, Renneboog and Zhao, 2011, Intintoli, Kahle, and Zhao, 2018). This leads to a director with network power holding 
a strong position in compensation negotiation. Consistent with this view, Hallock (1997) finds that in the U.S. CEOs reciprocally interlocked through directorships earn significantly higher compensation. Renneboog and Zhao (2011) find that in the U.K. well-connected CEOs earn higher compensation. Although not tested in China, the prior literature suggests that in China director compensation increases with director network power. Therefore, our hypothesis is:

H1: Directors with higher network prominence are more likely to be paid and receive higher compensation, ceteris paribus.

Renneboog and Zhao (2011) argue that a director network grants directors managerial power, which shields them from dismissal, predicting that better-connected directors have less turnover. Consistent with this view, Intintoli, Kahle, and Zhao (2018) find that, following misconduct, highly connected audit committee members are less likely to experience turnover than less-connected audit committee members. In contrast, an information advantage gained through director networks may provide new employment opportunities to directors. Thus, director networks might facilitate a director's departure from the current position to other outside options. Consistent with this view, Renneboog and Zhao (2018) find that better-connected directors experience higher turnover in the U.K.. Following Renneboog and Zhao (2018), we construct the hypothesis:

H2A: Directors with higher network prominence have higher labor mobility (measured by turnover), ceteris paribus.

A well-connected director may receive more compensation if the labor mobility is from a lower-paid to a higher-paid directorship. This leads to our next hypothesis:

H2B: Labour mobility of directors with high network prominence leads to higher total compensation, ceteris paribus.

Prior literature suggest that board connections improve firms' decision making and corporate governance (Larcker and Tayan, 2010, Renneboog and Zhao, 2011, Cai and Sevilir, 2012, Renneboog and Zhao, 2014, Intintoli, Kahle, and Zhao, 2018, Larcker, So, and Wang, 2013). Fama and Jensen (1983) argue that director effort may be rewarded in the labor market with additional future directorships. Consistent with Fama and Jensen (1983), Ferris, Jagannathan, and Pritchard (2003) find that directors acquire additional directorships after firm performance improvement. Likewise, Renneboog and Zhao (2018) suggest that director networks facilitate director access to labor market information. Thus, by accessing superior information in the labor market, a well-connected director is more likely to gain additional directorships. Thus, directors with high network prominence are more likely to gain additional directorships in the future, leading to the hypothesis:

H3A: Directors with high network prominence gain further board seats, ceteris paribus.

Additional directorships provide additional compensation and therefore increase the director total compensation in a given year. Therefore: 
H3B: Additional board seats gained through network prominence leads to higher total compensation, ceteris paribus.

Figure 3.1 demonstrates these hypotheses regarding network prominence and director compensation. H1 posits a direct effect of network prominence on directorship level compensation. H2 posits an indirect effect of network prominence on total compensation through labor mobility. H3 posits an indirect effect when network prominence leads to service on more boards, which leads to higher total compensation.

\subsection{Sample and variable construction}

\subsubsection{Sample}

Our sample consists of all firms listed on the Shanghai Stock Exchange (SSE) and Shenzhen Stock Exchange (SZSE) from 2005 to 2014. We start the sample from 2005 since directors' compensation information is not reported at the individual level until 2005. ${ }^{6}$ We collect the director profile, board profile and firm's ownership structure and accounting data from CSMAR (the Chinese Listed Firms Research Series database). ${ }^{7}$ We categorize our sample into independent directors and non-independent directors using the classification from the CSMAR database, which is a legal definition of director independence. ${ }^{8}$ In addition, we define executive directors as non-independent directors who hold executive positions in the firms and non-independent non-executive directors as non-independent directors who do not hold any executive positions in the firms. The director profile contains information on director compensation, turnover record, number of directorships and other director characteristics, such as the director's tenure, gender, age, shareholding and relationship to the large shareholders. The board profile contains information on board size, duality, ratio of independent directors, CEO compensation and number of board meetings. To minimize the influence of outliers, we winsorize firms' accounting data at the top and bottom $0.5 \%$ percentiles. Our final sample consists of 123,693 director-firm-year and 89,096 director-year observations. In our sample, the number of firms ranges from 1,374 in 2005 to 2,652 in 2014. In the following sections, we construct all variables. Table 3.14 defines all variables.

\footnotetext{
${ }^{6}$ The China Securities Regulatory Commission requires all listed firms to report compensation for each board of director beginning in 2005 .

${ }^{7}$ The CSMAR database is widely regarded as the most comprehensive and authoritative database to study corporate finance and corporate governance in Chinese listed firms. According to a report issued by ShenZhen GTA, the CSMAR database has been used in papers published in a dozen leading international journals including Journal of Finance, Journal of Financial Economics, Journal of Financial and Quantitative Analysis and Review of Financial Studies.

${ }^{8}$ See Section 1.2.3 for a detail discussion on the legal definition of director independence.
} 


\subsubsection{Dependent variables}

\section{Directorship level compensation}

We follow Chen and Keefe (2018) and measure the directorship level compensation by both the propensity to be paid and the level of compensation. To measure the propensity to be paid, we use the variable $\operatorname{Paid}(0 / 1)_{t}$ as the dependent variable. A value of 1 is assigned if a director receives compensation from a firm in a given year and 0 otherwise. Table 3.2 shows that $94.9 \%$ of independent directors, $98.1 \%$ of executive directors and $46 \%$ of nonindependent non-executive directors are paid. To measure the level of compensation, we use the variable $\operatorname{Ln}(\text { Compensation }+1)_{t}$ as the dependent variable. $L n(\text { Compensation }+1)_{t}$ is the natural logarithm of 1 plus the compensation that a director receives from a firm in a given year. In China, unpaid directors are common. Adding 1 to the compensation, we avoid losing too much of the sample during taking the natural logarithm. ${ }^{9}$ Table 3.2 shows that the average annual compensation is 60,382 CNY (equivalent to 8,879 USD with the exchange rate of $6.8 \mathrm{CNY} / \mathrm{USD}$ ) for an independent director, 525,681 CNY (equivalent to 77,306 USD with the exchange rate of $6.8 \mathrm{CNY/USD)}$ for an executive director and $170,829 \mathrm{CNY}$ (equivalent to 25,122 USD with the exchange rate of $6.8 \mathrm{CNY/USD)}$ for a non-independent non-executive director.

\section{Total director compensation}

We measure the total director compensation by $\operatorname{Ln}($ Total compensation +1$)$, which is the natural logarithm of 1 plus the aggregated compensation that a director collects from all firms that he or she serves in a given year. Table 3.2 shows that the average total compensation is $64,880 \mathrm{CNY}$ (equivalent to $9,541 \mathrm{USD}$ with the exchange rate of $6.8 \mathrm{CNY} / \mathrm{USD}$ ) for an independent director, 533,111 CNY (equivalent to 78,399 USD with the exchange rate of $6.8 \mathrm{CNY/USD)}$ for an executive director and 176,579 CNY (equivalent to 25,968 USD with the exchange rate of $6.8 \mathrm{CNY} / \mathrm{USD}$ ) for a non-independent non-executive director.

\section{Director turnover}

Following Yermack (2005), we measure director turnover by Turnover $(0 / 1)_{t}$, which is set to 1 for an observation in the year $t$ if a director does not appear in the annual report in the year $t+1$ and 0 otherwise. We exclude observations from delisted firms. We also exclude observations from directors leaving the board in year 6 or year 7 since there is term limit regulation in China. Table 3.2 shows that $14.5 \%$ of independent, $6.8 \%$ of executive and

\footnotetext{
${ }^{9}$ Alternatively, we re-run all regressions associated with director compensation by using the natural logarithm of director compensation as dependent variables. Because our results remain qualitatively unchanged, we do not report our results here. The results are available upon request.
} 
$13.6 \%$ of non-independent non-executive directors in the year $t$ leave their boards in the year $t+1$.

\section{Directors' future directorship}

To measure directors' ability to gain future directorships, we use the variable Directorship $p_{t+1}$ as the dependent variable. Directorship $p_{t+1}$ measures the number of directorships a director holds in the year $t+1$. To avoid double counting Directorship $p_{t+1}$ for directors with multiple directorships, we collapse director-firm-year observations into director-year observations. We report the summary statistics of Directorship $p_{t+1}$ in Table 3.2. On average, an independent director holds 1.56 directorships, an executive director holds 1.02 directorships and a non-independent non-executive director holds 1.12 directorships. In our sample, multiple directorships are common only for independent directors.

\subsubsection{Network prominence measure}

In social networks, social actors (such as individuals or organizations) build up links to other social actors, and the social actors and links form a network (Jackson, 2010). Therefore, a board network is a combination of a set of board of directors and connections through board interlocks. Figure 3.2 demonstrates the change of the independent director network in China from 2005 to 2014. In 2005, firms in the central part of the network are well-connected. However, firms in the periphery of the network are isolated from the network center. The independent director network in China becomes very connected by 2014, where almost all firms are connected through the independent director network. Figure 3.3 demonstrates the change of the non-independent director network in China from 2005 to 2014. In 2005, most firms are isolated from each other in the non-independent director network. By 2014, most firms are connected to each other in the central part of the network, but some firms in the periphery of the network are still isolated.

Eigenvector centrality takes into account both the number of an agent's connections and the number of connections of the agent's connections. In other words, eigenvector centrality measures both connections and the importance of these connections. Koka and Prescott (2008) suggest that eigenvector centrality measures network prominence since an agent in a prominent position in the network tends to occupy a central position therein. As such, we define Prominence $_{t}$ as equal to the eigenvector centrality of a director in the year $t$.

In Table 3.1, we tabulate a list of directors with the highest eigenvector centrality each year in our sample. Consistent with the fact that independent directors are generally more well-connected, seven of the ten most prominent directors are independent directors. Unsurprisingly, all these directors reside in Shanghai or Beijing, where most of the listed firms are located. Most of the directors in the list are academics from prestigious institutions 
in China. This finding is consistent with the frequency of academic directors in the Chinese independent directorship market. In addition, we find that network power coincides with both economic and political power. For example, in 2008 and 2009, the most prominent director LU Zhiqiang is a billionaire in China. In 2012 and 2013, the most prominent director ZHOU Qinye is the former vice president of Shanghai Stock Exchange.

\subsubsection{Control variables}

When studying the director compensation at directorship level, we control for director, board and firm features. The director level control variables include $\operatorname{Woman}(0 / 1), \operatorname{Age}_{t}$, Age $_{t}^{2}$, Tenure $_{t}$, Busy director $(0 / 1)_{t}, C E O / C O B(0 / 1)_{t}$, Related director $(0 / 1)_{t}$ and Ln(Share

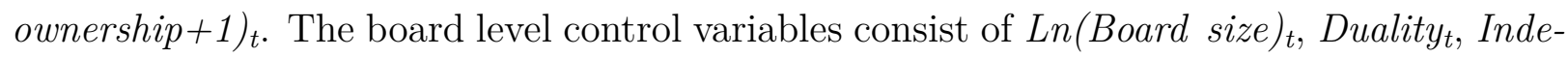
pendent director(\%) $)_{t}$, Meeting frequency $(\text { Firm })_{t}$ and $\operatorname{Ln}(C E O \text { compensation }+1)_{t}$. The firm level controls include State-owned(0/1) ${ }_{t}$, Largest shareholder(\%) $)_{t}$ Ln(Total Assets) $)_{t}$, Book leverage $_{t}$, Cash holdings ${ }_{t}, R O A_{t-1}$ and Stock volatility ${ }_{t-1}$.

When investigating the total compensation at director level, we use a similar set of control variables from previous regressions on director compensation at directorship level. However, we merge all directorship level controls into director level controls since the dependent variable $L n(\text { Total compensation }+1)_{t}$ is aggregated at the director level.

In the regressions on director turnover, we use a similar set of control variables as previous regressions on director compensation. To model the effect of compensation on turnover, we add $\operatorname{Ln}(\text { Compensation }+1)_{t}$ into the regression. To study the director's ability to gain future directorships, we use the same set of control variables from previous regressions on director turnover since those factors affecting turnover are likely to influence future directorships as well. However, we use the average values of several director level variables and all board and firm level variables since we merge director-firm-year observations into director-year observations.

In Table 3.2, we report the summary statistics of control variables. In China, $13.8 \%$ of independent directors, $11.1 \%$ of executive directors and $10 \%$ of non-independent nonexecutive directors are female. $29 \%$ of independent directors are busy directors. In our sample, the average independent director is 53.4 years old and has 6.1 years of board experience. The average executive director is 47.5 years old and has 5.4 years of board experience. The average non-independent non-executive director is 50.4 years old and has 6 years of board experience. In our sample, $19 \%$ of executive directors and $56 \%$ of nonindependent non-executive directors hold another position in the controlling shareholders' firms.

In China, the average board has nine members and $36.8 \%$ of them are independent directors. In one fifth of Chinese boards, the CEO and chairman are the same person. 
The average board meeting frequency is 9.3 per year. In our sample, $49.4 \%$ of firms are state-owned and the largest shareholders on average own $35.6 \%$ of the shares of the listed firm. The average firm has total book assets of 10.6 billion CNY (equivalent to 1.56 billion USD with the exchange rate of $6.8 \mathrm{CNY/USD),} \mathrm{book} \mathrm{leverage} \mathrm{of} 48 \%$ and cash holding of $17 \%$. On average, the ROA of Chinese listed firms is $3.6 \%$ and the annual stock volatility is $13.7 \%$. Table 3.3 provides a correlation matrix of key variables from regressions on director compensation. Panel A provides the pairwise correlation coefficients for independent directors. Panel B provides the pairwise correlation coefficient for executive directors. Panel $\mathrm{C}$ provides the pairwise correlation coefficient for non-independent non-executive directors. The correlation matrix denotes a positive correlation between network prominence and the level of compensation for all kinds of directors.

\subsection{Testing approach and results}

\subsubsection{Director network and directorship level compensation}

In this section, we explore whether network prominence increases directorship level compensation. The regressions control for year, industry, number of directorships, firm and director fixed effects. The unit of observation is a director-firm-year. Our estimation equation is as follows:

Director Compensation $_{i, f, t}=\alpha$ Network Measure $_{i, f, t-1}+\mathbf{X} \beta+\delta_{t}+\delta_{j}+\delta_{n}+\delta_{f}+\delta_{i}+\epsilon_{i, f, t}$

where $t$ represents the year, $j$ the industry, $n$ the number of directorships, $f$ the firm and $i$ the director. The dependent variable is either $\operatorname{Paid}(0 / 1)_{t}$ or $\operatorname{Ln}(\text { Compensation }+1)_{t}$. The variable of interest is Prominence ${ }_{i, f, t-1}$. $\mathbf{X}$ is a matrix of control variables previously described in Section 3.3.4. $\delta_{t}, \delta_{j}, \delta_{n}, \delta_{f}$ and $\delta_{i}$ denote year, industry, number of directorships, firm and director fixed effects respectively. $\epsilon_{i f t}$ is the error term. To control for potential serial correlation, we use robust standard errors adjusted for firm-level clustering and heteroskedasticity. We apply linear probability models to estimate Paid $(0 / 1)_{t}$ since the estimation of logit regressions with fixed effects reduces sample size. ${ }^{10}{ }^{11}$

Table 3.4 reports results of regressions investigating how network prominence affects directorship level compensation. We separate the sample of non-independent directors into executive directors and non-executive directors sub-samples. In Columns (1) and (2), we study

\footnotetext{
${ }^{10}$ In unreported tables, we estimate Paid $(0 / 1)_{t}$ using logit regressions with year, industry, number of directorships, firm and director fixed effects. The results from logit regressions are quantitatively similar to those from linear probability regressions. Therefore, our results are robust to the model selection.

${ }^{11}$ The logit regressions with fixed effects require within group variation for the dependent variables. Observations do not fit the requirement are deleted. The estimations of logit regressions with director fixed effects fail to converge due to the small sample size.
} 
the influence of network prominence on independent director compensation. In Columns (3) and (4), we investigate the influence of network prominence on non-independent executive director compensation. In Columns (5) and (6), we study the influence of network prominence on non-independent non-executive director compensation. In Columns (1), (3) and (5), the dependent variable is $\operatorname{Paid}(0 / 1)_{t}$. In Columns (2), (4) and (6), the dependent variable is $\operatorname{Ln}(\text { Compensation }+1)_{t}$. In Table 3.4, the regressions include year, industry and number of directorships fixed effects.

In Column (1), the coefficient associated with Prominence $_{t-1}$ is positive and statistically significant at the less than $1 \%$ level in explaining the propensity to receive compensation for independent directors. In Column (2), the coefficient associated with Prominence $_{t-1}$ is positive and statistically significant at the less than $1 \%$ level in explaining the level of compensation for independent directors. The above results support Hypothesis 1 that independent directors with higher network prominence are more likely to be paid and receive higher compensation. In Column (3), the coefficient associated with Prominence $_{t-1}$ is statistically no different than zero in explaining the propensity to receive compensation for executive directors. In Column (4), the coefficient associated with Prominence $_{t-1}$ is positive and statistically significant at the less than $1 \%$ level in explaining the level of compensation for executive directors. In Columns (5) and (6), the coefficients associated with Prominence $_{t-1}$ is statistically no different than zero in explaining the propensity to receive compensation and level of compensation for non-executive directors. These results suggest that the positive relationship between director network and compensation does not apply to non-executive directors.

Next, we include firm or director fixed effects into the previous regressions. Table 3.5 reports results of regressions on director compensation when firm and director fixed effects are included. In Panel A, the firm fixed effect controls for any time-invariant firm-specific factors related to both network prominence and director compensation. In Panel B, the director fixed effects control for any time-invariant director-specific factors related to both network prominence and director compensation. In Columns (1) and (2), the coefficients associated with Prominence $_{t-1}$ are positive and statistically significant at the less than $1 \%$ level in explaining $\operatorname{Paid}(0 / 1)_{t}$ and $\operatorname{Ln}(\text { Compensation }+1)_{t}$ for independent directors. This result suggests that the positive relationship between network prominence and independent director compensation is robust to firm and director fixed effects. In addition, in Column (4), the coefficients associated with Prominence $_{t-1}$ are statistically no different than zero in explaining $\operatorname{Ln}(\text { Compensation }+1)_{t}$ for executive directors. Therefore, the positive relationship between network prominence and executive director compensation from Table 3.4 is driven by omitted variables in firm and director levels. 


\subsubsection{Director network and director turnover}

In this section, we estimate linear probability models regarding the effects of network prominence on director turnover. ${ }^{12}$ The regressions control for year, industry, number of directorships, firm and director fixed effects. The unit of observation is a director-firm-year. Our estimation equation is as follows:

$$
\text { Turnover }_{i, f, t}=\alpha \text { Network Measure }_{i, f, t}+\mathbf{X} \beta+\delta_{t}+\delta_{j}+\delta_{n}+\delta_{f}+\delta_{i}+\epsilon_{i, f, t}
$$

where $t$ represents the year, $j$ the industry, $n$ the number of directorships, $f$ the firm and $i$ the director. The dependent variable is Turnover $(0 / 1)_{t}$. The variable of interest is Prominence $_{i, f, t}$. $\mathbf{X}$ is a matrix of control variables previously described in Section 3.3.4. $\delta_{t}, \delta_{j}, \delta_{n}, \delta_{f}$ and $\delta_{i}$ denote year, industry, number of directorships, firm and director fixed effects respectively. $\epsilon_{i f t}$ is the error term. To control for potential serial correlation, we use robust standard errors adjusted for firm-level clustering and heteroskedasticity.

Table 3.6 reports results of regressions investigating how network prominence affects director turnover. In Column (1), we examine the influence of network prominence on independent director turnover. In Column (2), we study the influence of network prominence on executive director turnover. In Column (3), we examine the impact of network prominence on non-independent non-executive director turnover. In Table 3.6, the regressions include year, industry and number of directorships fixed effects. In Columns (1), (2) and (3), the

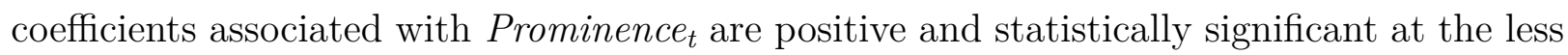
than $1 \%$ level in explaining Turnover $(0 / 1)_{t}$ for all directors. The above results support Hypothesis H2A that directors with higher network prominence have higher labor mobility.

Table 3.7 reports results of regressions on director turnover when firm and director fixed effects are included. In Columns (1), (2) and (3), the coefficients associated with Prominence $_{t}$ are positive and statistically significant at the less than $1 \%$ level in explaining Turnover $(0 / 1)_{t}$. Therefore, the positive relationship between network prominence and director turnover is robust to firm and director fixed effects.

\footnotetext{
${ }^{12}$ See discussion in Section 3.4.1 on the reason for linear probability models. In unreported tables, we estimate Turnover $(0 / 1)_{t}$ using logit regressions with year, industry, number of directorships, firm and director fixed effects. The results from logit regressions are quantitatively similar to those from linear probability regressions.
} 


\subsubsection{Director network and further directorship}

In this section, we investigate whether network prominence improves directors' ability to gain more future directorships. The regressions control for year and director fixed effects. The unit of observation is a director-year. Our estimation equation is as follows:

$$
\text { Number of directorships }_{i, t+1}=\alpha \text { Network } \text { Measure }_{i, t}+\mathbf{X} \beta+\delta_{t}+\delta_{i}+\epsilon_{i, t}
$$

where $t$ represents the year and $i$ the director. The dependent variable is Directorship $p_{t+1}$. The variable of interest is Prominence $_{i, t}$. $\mathbf{X}$ is a matrix of control variables previously described in Section 3.3.4. $\delta_{t}$ and $\delta_{i}$ denote year and director fixed effects respectively. $\epsilon_{i t}$ is the error term. To control for potential serial correlation, we use robust standard errors adjusted for director-level clustering and heteroskedasticity.

Table 3.8 reports results of regressions investigating how network prominence affects directors' ability to gain more future directorships. In Column (1), we study the impact of network prominence on independent directors' ability to gain more future directorships. In Column (2), we examine the influence of network prominence on non-independent executive directors' ability to gain more future directorships. In Column (3), our studies investigate the impact of network prominence on non-independent non-executive directors' ability to gain more future directorships. In Table 3.8, the regressions include year fixed effects. In Columns (1), (2) and (3), the coefficients associated with Prominence $_{t}$ are positive and statistically significant at the less than $1 \%$ level in explaining Directorship $p_{t+1}$. The above results support Hypothesis H3A that directors with higher network prominence gain further board seats.

Table 3.9 reports results of regressions on directors' ability to gain more future board seats when director fixed effects are included. In Columns (1), (2) and (3), the coefficients associated with Prominence $_{t}$ are positive and statistically significant at the less than $5 \%$ level in explaining Directorship Dit $_{1}$. Thus, the positive relationship between network prominence and directors' ability to gain further board seats is robust to director fixed effects.

\subsubsection{Director network on total director compensation through labor mobility}

In this section, we investigate whether network prominence increases total director compensation through labor mobility. The unit of observation is a director-year since we calculate the total compensation through aggregating the compensation that directors receive from 
each firm that they serve in a given year. In regressions, we control for year and director fixed effects. Our estimation equation is as follows:

Total director compensation $_{i, t}=\alpha_{\mathbf{1}}$ Turnover $_{i, t-1}+\alpha_{\mathbf{2}}$ Network $_{\text {Measure }}$ (t,t-1 $+\alpha_{\mathbf{3}}$ Turnover $_{i, t-1} *$ Network $_{\text {Measure }}^{i, t-1}+\alpha_{\mathbf{4}}$ Number of directorship $_{i, t}+\mathbf{X} \beta+\delta_{t}+\delta_{i}+\epsilon_{i, t}$

where $t$ represents the year and $i$ the director. The dependent variable is $L n(\text { Total compensation }+1)_{t}$. The variables of interest are Turnover $(\%)_{t-1}$, Prominence Pr- $_{1}$ and its interaction term. A positive (negative) interaction term between Turnover $(\%)_{t-1}$ and Prominence $_{t-1}$ tests hypothesis $\mathrm{H} 2 \mathrm{~B}$ that network prominence in the year $t-1$ increases (decreases) total compensation in the year $t$ through director turnover in the year $t-1$. The coefficient associated with

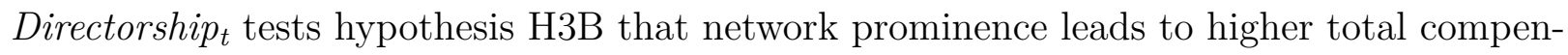
sation through more board seats. $\mathbf{X}$ is a matrix of control variables previously described in Section 3.3.4. $\delta_{t}$ and $\delta_{i}$ denote year and director fixed effects respectively. $\epsilon_{i t}$ is the error term. To control for potential serial correlation, we use robust standard errors adjusted for director-level clustering and heteroskedasticity.

Table 3.10 reports results of regressions investigating whether network prominence increases total compensation through director turnover. In Column (1), the coefficient associated with Turnover $(\%)_{t-1}$ is negative and statistically significant at the less than $1 \%$ level in explaining total compensation for independent directors. This result suggests that an independent director receives less total compensation in the year $t$ if he or she experiences turnover in the year $t-1$. In Column (1), the coefficient associated with Prominence $_{t-1}$ is positive and statistically significant at the less than $1 \%$ level in explaining total compensation for independent directors, suggesting that network prominence increases the total compensation for independent directors. In Column (1), the coefficient associated with the interaction term between Turnover $(\%)_{t-1}$ and Prominence $t_{t-1}$ is positive and statistically significant at the less than $1 \%$ level in explaining total compensation for independent directors. The result from the interaction term supports hypothesis $\mathrm{H} 2 \mathrm{~B}$ that the network prominence of independent directors may increase their total compensation through director turnover.

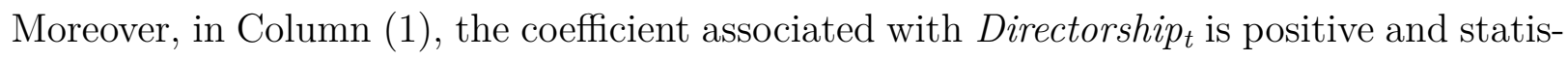
tically significant at the less than $5 \%$ level in explaining total compensation for independent directors. This result is consistent with hypothesis H3B that network prominence increases total compensation through more board seats. 


\subsection{Robustness}

\subsubsection{Related director}

Related directors are non-independent directors holding positions in both the listed firms and controlling firms. ${ }^{13}$ Their relationship with controlling shareholders may influence their career outcomes. ${ }^{14}$ The literature on related directors' career outcomes is scant since related directors are uncommon in western countries. However, related directors are very common in China, where $19 \%$ of executive directors and $56 \%$ of non-independent non-executive directors in our sample are related directors.

Table 3.12 reports results of regressions examining the interaction term between Related director $(0 / 1)_{t}$ and Prominence ${ }_{t}$ in explaining Turnover $(0 / 1)_{t}$ for non-independent directors. In Columns (1) and (2), the coefficients associated with Related director $(0 / 1)_{t}$ are negative and statistically significant at the less than $1 \%$ level in explaining Turnover $(0 / 1)_{t}$, suggesting that non-independent directors holding another position in the controlling firms (related directors) are less likely to experience turnover. This result suggests that the relationship with controlling shareholders increases non-independent directors' job security. In addition, in Column (2), the coefficients associated with the interaction term between Related

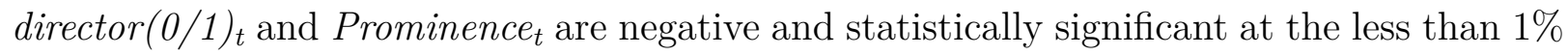
level in explaining Turnover $(0 / 1)_{t}$ for non-independent non-executive directors. Therefore, board connections increase job security for related directors.

Table 3.13 reports results of regressions examining the interaction term between Related director $(0 / 1)_{t}$ and Prominence Pin explaining Directorship $_{t+1}$ for non-independent directors. In Column (2), the coefficient associated with Related director $(0 / 1)_{t}$ is positive and statistically significant at the less than $10 \%$ level in explaining Directorship $p_{t+1}$ for non-independent non-executive directors. This result suggests that non-independent non-executive directors holding another position in the controlling firms (related directors) are likely to gain more future directorships at the next period. Moreover, in Column (2), the coefficient associated with the interaction term between Related director $(0 / 1)_{t}$ and Prominence $_{t}$ is positive and statistically significant at the less than $1 \%$ level in explaining Directorship $p_{t+1}$, indicating that network prominence increases the ability to gain further board seats for non-independent non-executive directors related to controlling shareholders.

\footnotetext{
${ }^{13}$ China Securities Regulatory Commission (CSRC) forbids an individual holds a position in a controlling firm to serve as an independent director in the listed firm. Thus, related directors can only hold nonindependent directorships.

${ }^{14}$ For example, Lo, Wong, and Firth (2010) suspect but do not test that a related director is less likely to be paid and receives less compensation as controlling shareholders may pay part or all of director compensation. Chen and Keefe (2018) empirically test and find that in China related directors are less likely to be paid and receive less compensation.
} 


\subsection{Conclusion}

Through board networks, well-connected directors become more informed. Previous studies find that this information advantage benefits directors' careers on western boards. However, there is no research on whether this effect holds for the directors in China, where the ownership structure and governance issues differ from those in the U.S. and U.K. (Jiang and Kim, 2015). Therefore, our study of board networks on directors' career outcomes in China fills this gap.

Our study suggests that director networks are positively priced in independent director compensation. Our findings on independent director compensation are consistent with the literature suggesting that board network prominence signals director quality (Fama and Jensen, 1983, Renneboog and Zhao, 2011, Intintoli, Kahle, and Zhao, 2018).

Except for compensation, board networks increase director turnover. This result suggests that board networks provide directors more new employment opportunities, increasing their job mobility. In contrast, we find that related directors experience less turnover than non-related directors, suggesting that the relationship with controlling shareholders may shield directors from dismissal. Moreover, related directors with more board connections experience less turnover than those with fewer board connections, suggesting that board connections could increase job security for related directors. The mixed results of board connections on director turnover are not surprising. Through director networks, non-related directors could get more information on outside employment opportunities, and related directors could gain managerial power to protect them from dismissal.

Our study suggests that well-connected directors receive more future directorships. This finding supports the argument that well-connected directors are rewarded with more future directorships due to either their quality or superior information in the labor market (Larcker and Tayan, 2010, Renneboog and Zhao, 2011, Cai and Sevilir, 2012, Renneboog and Zhao, 2014, Intintoli, Kahle, and Zhao, 2018, Larcker, So, and Wang, 2013, Fama and Jensen, 1983, Ferris, Jagannathan, and Pritchard, 2003, Renneboog and Zhao, 2018). Moreover, we find that related directors gain more future directorships than non-related directors, suggesting that the relationship with controlling shareholders benefits related directors' careers. Furthermore, we find that related directors with more board connections receive more future directorships.

Our study identifies channels where network prominence indirectly increases total compensation. For example, well-connected independent directors may receive higher total compensation through moving from low-paid directorships to high-paid directorships (turnover channel). In addition, they may increase total compensation from holding more board seats. Overall, we find that the board network directly increases directorship level compensation 
and indirectly leads to higher total compensation through labor mobility and additional board seats. 


\section{Bibliography}

Cai, Ye, and Merih Sevilir, 2012, Board connections and M\&A transactions, Journal of Financial Economics 103, 327-349.

Chen, Zonghao, and Michael O'Connor Keefe, 2018, Board of director compensation in China: To pay or not to pay? How much to pay?, Emerging Markets Review 37, 66-82.

Fama, Eugene F., and Michael C. Jensen, 1983, Separation of ownership and control, The Journal of Law \& Economics 26, 301-325.

Ferris, Stephen P., Javakhadze David, and Liu Yun, 2016, The corporate demand for external connectivity: Pricing boardroom social capital, Available at SSRN.

Ferris, Stephen P., Murali Jagannathan, and A. C. Pritchard, 2003, Too busy to mind the business? Monitoring by directors with multiple board appointments, The Journal of Finance 58, 1087-1111.

Hallock, Kevin F., 1997, Reciprocally interlocking boards of directors and executive compensation, Journal of Financial and Quantitative Analysis 32, 331-344.

Intintoli, Vincent J., Kathleen M. Kahle, and Wanli Zhao, 2018, Director connectedness: Monitoring efficacy and career prospects, Journal of Financial and Quantitative Analysis 53, 65-108.

Jackson, Matthew O., 2010, Social and economic networks (Princeton university press).

Jiang, Fuxiu, and Kenneth A. Kim, 2015, Corporate governance in China: A modern perspective, Journal of Corporate Finance 32, 190-216.

Koka, Balaji R., and John E. Prescott, 2008, Designing alliance networks: the influence of network position, environmental change, and strategy on firm performance, Strategic Management Journal 29, 639-661.

Larcker, David F., Eric C. So, and Charles C. Y. Wang, 2013, Boardroom centrality and firm performance, Journal of Accounting and Economics 55, 225-250.

Larcker, David F., and Brian Tayan, 2010, Good for the director, good for shareholders, Available at SSRN.

Lo, Agnes WY, Raymond MK Wong, and Michael Firth, 2010, Can corporate governance deter management from manipulating earnings? evidence from related-party sales transactions in China, Journal of Corporate Finance 16, 225-235. 
Renneboog, Luc, and Yang Zhao, 2011, Us knows us in the UK: On director networks and CEO compensation, Journal of Corporate Finance 17, 1132-1157.

2014, Director networks and takeovers, Journal of Corporate Finance 28, 218-234.

, 2018, Director networks, turnover, and appointments, Available at SSRN.

Yermack, David, 2005, Remuneration, retention, and reputation incentives for outside directors, The Journal of Finance 59, 2281-2308. 


\subsection{Tables}

Figure 3.1: Hypothesis of network power on director compensation

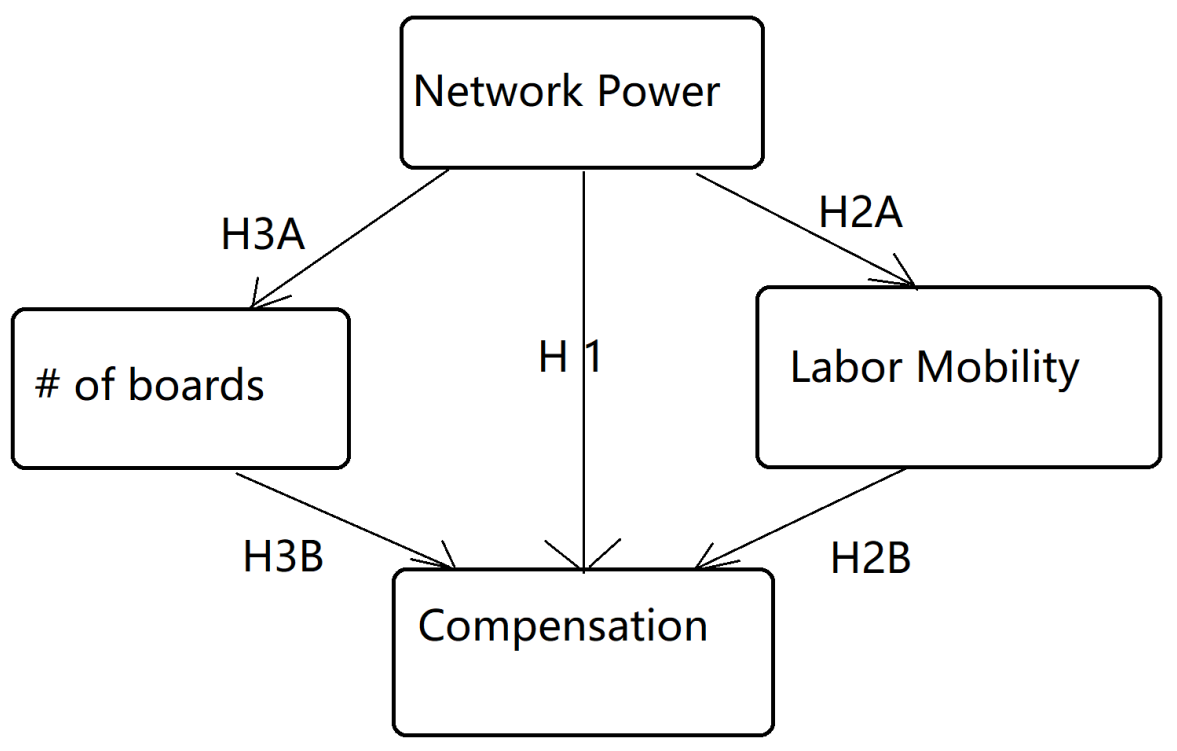


Figure 3.2: Independent director network from 2005 to 2014
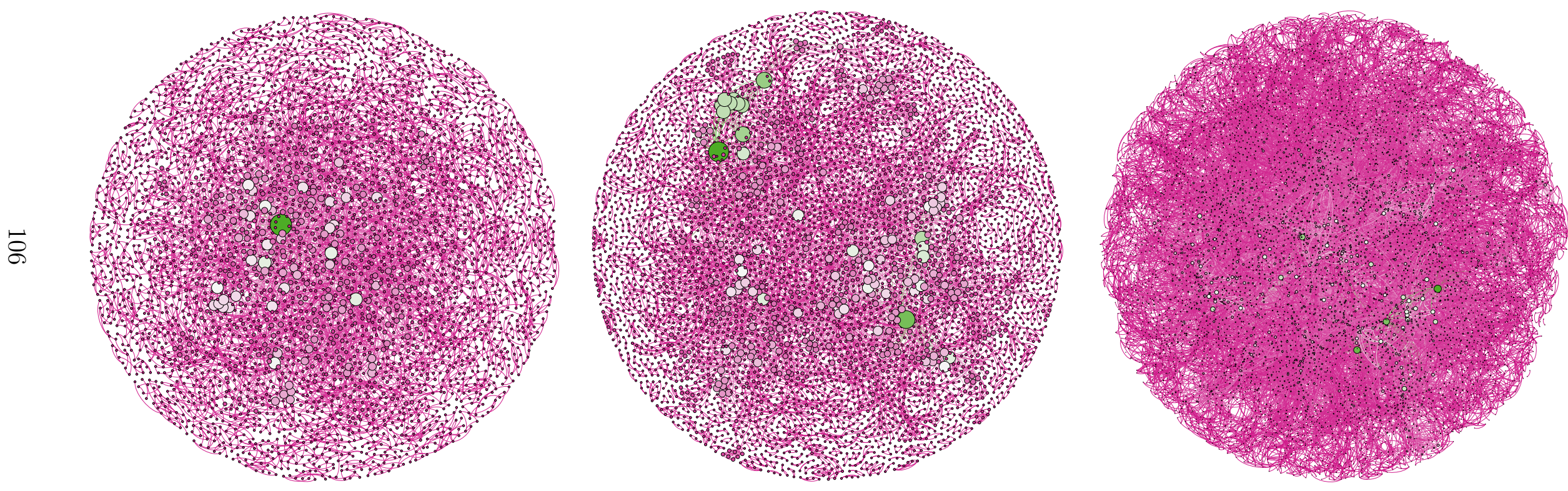

(a) Independent director network at 2005

(b) Independent director network at 2009

(c) Independent director network at 2014 
Figure 3.3: Non-independent director network from 2005 to 2014
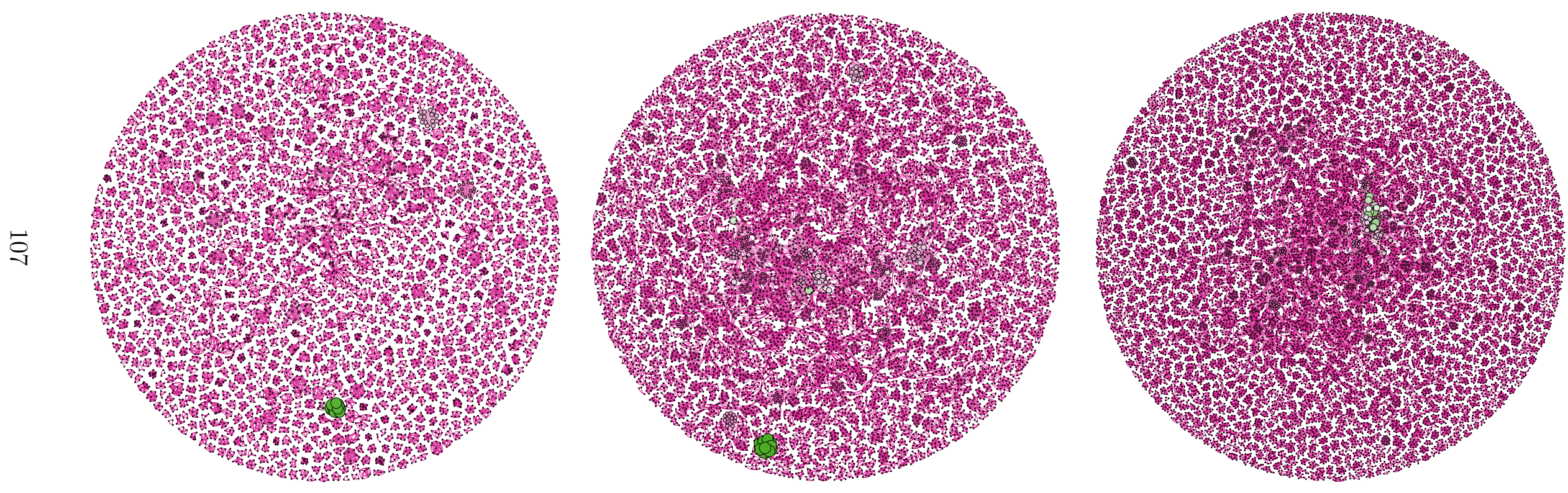

(a) Non-independent director network at 2005

(b) Non-independent director network at 2009

(c) Non-independent director network at 2014 
Table 3.1: Board of directors with most network prominence each year

This table reports the names, number of directorships, network prominence and profile for the board of directors with most network prominence each year from 2005 to 2014 .

\begin{tabular}{|c|c|c|c|c|c|}
\hline Year & $\begin{array}{l}\text { Director } \\
\text { name }\end{array}$ & $\begin{array}{l}\text { Multiple } \\
\text { directorships }\end{array}$ & $\begin{array}{l}\text { Network } \\
\text { prominence }\end{array}$ & $\begin{array}{l}\text { Director } \\
\text { type }\end{array}$ & $\begin{array}{l}\text { Director } \\
\text { profile }\end{array}$ \\
\hline 2005 & WANG Fanghua & 6 & 12.9 & Independent & $\begin{array}{l}\text { WANG Fanghua is the professor in market- } \\
\text { ing at Antai School of Finance and Economics, } \\
\text { Shanghai Jiaotong University. }\end{array}$ \\
\hline 2006 & LI Yang & 5 & 9.6 & Independent & $\begin{array}{l}\text { LI Yang is the director of The Financial Re- } \\
\text { search Institution, Chinese Academy of Social } \\
\text { Sciences. }\end{array}$ \\
\hline 2007 & ZHANG Jianwei & 4 & 11.4 & Non-independent & $\begin{array}{l}\text { ZHANG Jianwei is the vice president of the } \\
\text { Shanghai Jiushi Group, which is the share- } \\
\text { holder of all listed firms where he sits at. }\end{array}$ \\
\hline 2008 & LU Zhiqiang & 3 & 9.7 & Non-independent & $\begin{array}{l}\text { LU Zhiqiang is a billionaire in China. At } 2009 \text {, } \\
\text { he is ranked the fifth richest person in China } \\
\text { by Rupert Hoogewerf. He is the shareholder of } \\
\text { all these three firms. }\end{array}$ \\
\hline 2009 & LU Zhiqiang & 3 & 12.9 & Non-independent & $\begin{array}{l}\text { LU Zhiqiang is a billionaire in China. At } 2009 \text {, } \\
\text { he is ranked the fifth richest person in China } \\
\text { by Rupert Hoogewerf. He is the shareholder of } \\
\text { all these three firms. }\end{array}$ \\
\hline 2010 & GAO Peiyong & 3 & 8.7 & Independent & $\begin{array}{l}\text { GAO Peiyong is the director of the Na- } \\
\text { tional Academy of Economic Strategy, Chinese } \\
\text { Academy of Social Sciences. }\end{array}$ \\
\hline 2011 & WU Xiaoqiu & 6 & 17.5 & Independent & $\begin{array}{l}\text { WU Xiaoqiu is a professor at the School of } \\
\text { Finance, Renming University. }\end{array}$ \\
\hline 2012 & ZHOU Qinye & 6 & 8 & Independent & $\begin{array}{l}\text { ZHOU Qinye served as the vice president of } \\
\text { the Shanghai Stock Exchange before } 2012 \text {. Be- } \\
\text { tween } 2011 \text { and } 2012 \text {, he served as the chief } \\
\text { accountant of the Shanghai Stock Exchange. }\end{array}$ \\
\hline 2013 & ZHOU Qinye & 9 & 6.6 & Independent & $\begin{array}{l}\text { ZHOU Qinye served as the vice president of } \\
\text { the Shanghai Stock Exchange before } 2012 \text {. Be- } \\
\text { tween } 2011 \text { and } 2012 \text {, he served as the chief } \\
\text { accountant of the Shanghai Stock Exchange. }\end{array}$ \\
\hline 2014 & LV Changjiang & 7 & 7.2 & Independent & $\begin{array}{l}\text { LV Changjiang is the accounting professor at } \\
\text { the School of Management, Fudan University }\end{array}$ \\
\hline
\end{tabular}


Table 3.2: Summary statistics

This table provides the summary statistics for all variables. Table 3.14 defines all variables. Panel A provides the summary statistics for independent directors. Panel B provides the summary statistics for executive directors. Panel C provides the summary statistics for non-independent non-executive directors. Panel D provides the summary statistics for board and firm characteristics in firm-year. All monetary terms are denominated in Chinese Yuan (CNY).

\begin{tabular}{|c|c|c|c|c|c|c|}
\hline & Obs & Mean & $\mathrm{SD}$ & 25 th & Median & 75 th \\
\hline \multicolumn{7}{|l|}{ Panel A. Independent director characteristics } \\
\hline $\operatorname{Paid}(0 / 1)_{t}$ & 49,442 & 0.949 & 0.22 & 1 & 1 & 1 \\
\hline Director compensation (Thousands $C N Y)_{t}$ & 49,442 & 60.382 & 61.246 & 36 & 50 & 70 \\
\hline Total compensation (Thousands $C N Y)_{t}$ & 29,764 & 64.88 & 111.084 & 38 & 50 & 70 \\
\hline Turnover $(0 / 1)_{t}$ & 45,417 & 0.145 & 0.352 & 0 & 0 & 0 \\
\hline Directorshipt+1 $_{t+1}$ & 29,779 & 1.564 & 0.987 & 1 & 1 & 2 \\
\hline Prominencet-1 & 49,442 & 0.733 & 0.959 & 0.158 & 0.396 & 0.94 \\
\hline $\operatorname{Woman}(0 / 1)$ & 49,442 & 0.138 & 0.345 & 0 & 0 & 0 \\
\hline Busy director $(0 / 1)_{t}$ & 49,442 & 0.29 & 0.454 & 0 & 0 & 1 \\
\hline Tenure $_{t}$ & 49,442 & 6.11 & 3.218 & 3 & 6 & 8 \\
\hline $\mathrm{Age}_{t}$ & 49,442 & 53.405 & 9.709 & 46 & 51 & 61 \\
\hline \multicolumn{7}{|l|}{ Panel B. Executive director characteristics } \\
\hline $\operatorname{Paid}(0 / 1)_{t}$ & 26,498 & 0.981 & 0.135 & 1 & 1 & 1 \\
\hline Director compensation(Thousands $C N Y)_{t}$ & 26,498 & 525.681 & 649.279 & 220 & 373.3 & 615.7 \\
\hline Total compensation(Thousands $C N Y)_{t}$ & 22,350 & 533.111 & 651.772 & 221.8 & 380 & 628.8 \\
\hline Turnover $(0 / 1)_{t}$ & 24,819 & 0.068 & 0.251 & 0 & 0 & 0 \\
\hline Directorship $_{t+1}$ & 22,227 & 1.017 & 0.159 & 1 & 1 & 1 \\
\hline Prominencet-1 & 26,498 & 0.341 & 0.422 & 0.11 & 0.22 & 0.411 \\
\hline $\operatorname{Woman}(0 / 1)$ & 26,498 & 0.111 & 0.315 & 0 & 0 & 0 \\
\hline Tenuret & 26,498 & 5.432 & 3.212 & 3 & 4 & 7 \\
\hline Aget $_{t}$ & 26,498 & 47.471 & 6.58 & 43 & 47 & 52 \\
\hline Related director $(0 / 1)_{t}$ & 26,498 & 0.19 & 0.392 & 0 & 0 & 0 \\
\hline Share ownership(Millions Shares $)_{t}$ & 26,498 & 6.348 & 30.11 & 0 & 0 & 0.511 \\
\hline \multicolumn{7}{|c|}{ Panel C. Non-independent non-executive director characteristics } \\
\hline $\operatorname{Paid}(0 / 1)_{t}$ & 47,753 & 0.46 & 0.498 & 0 & 0 & 1 \\
\hline Director compensation (Thousands $C N Y)_{t}$ & 47,753 & 170.829 & 477.699 & 0 & 0 & 165.9 \\
\hline Total compensation(Thousands $C N Y)_{t}$ & 36,982 & 176.579 & 480.464 & 0 & 0 & 180 \\
\hline Turnover $(0 / 1)_{t}$ & 45,831 & 0.136 & 0.343 & 0 & 0 & 0 \\
\hline Directorship $_{t+1}$ & 37,014 & 1.119 & 0.427 & 1 & 1 & 1 \\
\hline Prominencet-1 & 47,753 & 0.486 & 0.704 & 0.134 & 0.273 & 0.569 \\
\hline $\operatorname{Woman}(0 / 1)$ & 47,753 & 0.1 & 0.3 & 0 & 0 & 0 \\
\hline Tenuret & 47,753 & 6.015 & 3.456 & 3 & 5 & 8 \\
\hline Aget $_{t}$ & 47,753 & 50.431 & 7.795 & 45 & 50 & 56 \\
\hline Related director $(0 / 1)_{t}$ & 47,753 & 0.56 & 0.496 & 0 & 1 & 1 \\
\hline Share ownership(Millions Shares $)_{t}$ & 47,753 & 4 & 31.241 & 0 & 0 & 0 \\
\hline \multicolumn{7}{|l|}{ Panel D. Board and firm characteristics } \\
\hline Meeting frequency $(\text { Firm })_{t}$ & 14,008 & 9.349 & 3.752 & 7 & 9 & 11 \\
\hline$C E O$ compensation (Thousands $C N Y)_{t}$ & 14,008 & 548.809 & 714.973 & 193.001 & 390.001 & 667.201 \\
\hline Board sizet & 14,008 & 9.032 & 1.937 & 8 & 9 & 9 \\
\hline Duality $_{t}$ & 14,008 & 0.205 & 0.404 & 0 & 0 & 0 \\
\hline Independent director $(\%)_{t}$ & 14,008 & 0.368 & 0.054 & 0.333 & 0.333 & 0.4 \\
\hline State-owned $(0 / 1)_{t}$ & 14,008 & 0.494 & 0.5 & 0 & 0 & 1 \\
\hline Largest shareholder $(\%)_{t}$ & 14,008 & 0.356 & 0.155 & 0.233 & 0.337 & 0.468 \\
\hline Total assets $(\text { Billions } C N Y)_{t}$ & 14,008 & 10.578 & 30.307 & 1.235 & 2.613 & 6.461 \\
\hline Book leverage $_{t}$ & 14,008 & 0.48 & 0.235 & 0.306 & 0.481 & 0.642 \\
\hline Cash holdingst & 14,008 & 0.17 & 0.297 & 0.065 & 0.12 & 0.219 \\
\hline$R O A_{t}$ & 14,008 & 0.036 & 0.06 & 0.012 & 0.033 & 0.063 \\
\hline Stock volatilityt & 13,962 & 0.137 & 0.088 & 0.097 & 0.123 & 0.161 \\
\hline
\end{tabular}


Table 3.3: Cross correlations of network prominence and board of director compensation

This table provides the correlation matrix of key variables from regressions on directors' compensation. Panel A reports the correlation matrix for independent director compensation. Panel B reports the correlation matrix for executive director compensation. Panel C reports the correlation matrix for non-independent non-executive director compensation. Table 3.14 provides all variable definitions. Superscripts ${ }^{*},{ }^{*}$ and ${ }^{* * *}$ denote significance at the $10 \%, 5 \%$ and $1 \%$ levels, respectively.

\begin{tabular}{|c|c|c|c|c|c|c|c|c|c|c|}
\hline Panel A: Independent director & $(1)$ & $(2)$ & (3) & $(4)$ & $(5)$ & $(6)$ & $(7)$ & & & \\
\hline (1)Paid $(0 / 1)_{t}$ & 1 & & & & & & & & & \\
\hline (2)Director compensation(Thousands $C N Y)_{t}$ & $0.263^{* *}$ & 1 & & & & & & & & \\
\hline (3) Prominencet-1 & 0.00641 & $0.147^{* *}$ & 1 & & & & & & & \\
\hline (4) Directorshipt & $0.0217^{* *}$ & $0.0409 * *$ & $0.506^{* *}$ & 1 & & & & & & \\
\hline (5) $\operatorname{Woman}(0 / 1)$ & -0.00735 & $-0.0251^{* *}$ & $-0.0720^{* *}$ & $-0.0474^{* *}$ & 1 & & & & & \\
\hline (6) Age $_{t}$ & -0.00199 & $0.0985^{* *}$ & $0.0446^{* *}$ & $-0.00995^{*}$ & $-0.0950^{* *}$ & 1 & & & & \\
\hline (7) Tenure $_{t}$ & $0.104^{* *}$ & $0.0621^{* *}$ & 0.00979 & $0.0615^{* *}$ & $-0.0224^{* *}$ & $0.137^{* *}$ & 1 & & & \\
\hline Panel B: Executive director & $(1)$ & $(2)$ & (3) & (4) & $(5)$ & (6) & (7) & $(8)$ & (9) & $(10)$ \\
\hline (1)Paid $(0 / 1)_{t}$ & 1 & & & & & & & & & \\
\hline (2) Director compensation (Thousands $C N Y)_{t}$ & $0.107^{* *}$ & 1 & & & & & & & & \\
\hline (3) Prominencet-1 & $-0.0271^{* *}$ & $0.254^{* *}$ & 1 & & & & & & & \\
\hline (4) Directorshipt & $-0.0353^{* *}$ & $0.0484^{* *}$ & $0.246^{* *}$ & 1 & & & & & & \\
\hline (5) $\operatorname{Woman}(0 / 1)$ & $0.0192^{* *}$ & $-0.0234^{* *}$ & $-0.0318^{* *}$ & $-0.0164^{* *}$ & 1 & & & & & \\
\hline (6) $A g e_{t}$ & -0.00774 & $0.125^{* *}$ & $0.0779^{* *}$ & $0.0298^{* *}$ & $-0.0611^{* *}$ & 1 & & & & \\
\hline (7) Tenure $_{t}$ & $0.0375^{* *}$ & $0.0966^{* *}$ & $0.0268^{* *}$ & $0.0783^{* *}$ & $-0.0334^{* *}$ & $0.279^{* *}$ & 1 & & & \\
\hline (8) $C E O / C O B(0 / 1)_{t}$ & 0.00114 & $0.124^{* *}$ & 0.00404 & $0.0384^{* *}$ & $-0.142^{* *}$ & $0.129 * *$ & $0.0962^{* *}$ & 1 & & \\
\hline (9) Related director $(0 / 1)_{t}$ & $-0.0848^{* *}$ & $0.0290^{* *}$ & 0.0131 & $0.0454^{* *}$ & $-0.0266^{* *}$ & $0.0822^{* *}$ & $0.0876^{* *}$ & $0.184^{* *}$ & 1 & \\
\hline (10) $L n(\text { Share ownership }+1)_{t}$ & $0.0876^{* *}$ & $0.0749^{* *}$ & $-0.0734^{* *}$ & $-0.0266^{* *}$ & $0.0138^{*}$ & $0.0569 * *$ & -0.00372 & $0.0390^{* *}$ & $-0.0340^{* *}$ & 1 \\
\hline Panel C: Non-executive director & $(1)$ & $(2)$ & (3) & $(4)$ & $(5)$ & (6) & $(7)$ & (8) & (9) & $(10)$ \\
\hline$(1) \operatorname{Paid}(0 / 1)_{t}$ & 1 & & & & & & & & & \\
\hline (2)Director compensation(Thousands $C N Y)_{t}$ & $0.385^{* *}$ & 1 & & & & & & & & \\
\hline (3) Prominencet-1 & $-0.0856^{* *}$ & $0.0122^{*}$ & 1 & & & & & & & \\
\hline (4) Directorship & $-0.0957^{* *}$ & $-0.0552^{* *}$ & $0.441^{* *}$ & 1 & & & & & & \\
\hline (5) $\operatorname{Woman}(0 / 1)$ & 0.00552 & $-0.0301^{* *}$ & $-0.0265^{* *}$ & $-0.0249 * *$ & 1 & & & & & \\
\hline (6) $\mathrm{Aget}_{\mathrm{t}}$ & $0.0366^{* *}$ & $0.0946^{* *}$ & $0.0555^{* *}$ & $0.0202^{* *}$ & $-0.111^{* *}$ & 1 & & & & \\
\hline (7) Tenuret & $0.0863^{* *}$ & $0.144^{* *}$ & -0.000347 & $0.0250^{* *}$ & $-0.0413^{* *}$ & $0.286^{* *}$ & 1 & & & \\
\hline (8) $C E O / C O B(0 / 1)_{t}$ & $0.177^{* *}$ & $0.259^{* *}$ & $-0.0425^{* *}$ & $-0.0238^{* *}$ & $-0.0927^{* *}$ & $0.126^{* *}$ & $0.146^{* *}$ & 1 & & \\
\hline (9) Related director $(0 / 1)_{t}$ & $-0.289^{* *}$ & $-0.112^{* *}$ & $0.0938^{* *}$ & $0.0610^{* *}$ & -0.00692 & 0.00284 & $0.0521^{* *}$ & $0.0764^{* *}$ & & \\
\hline$(10) \operatorname{Ln}(\text { Share ownership }+1)_{t}$ & $0.261^{* *}$ & $0.227^{* *}$ & $-0.0937^{* *}$ & $-0.0914^{* *}$ & $0.0166^{* *}$ & $0.0999 * *$ & $0.116^{* *}$ & $0.188^{* *}$ & $-0.186^{* *}$ & 1 \\
\hline
\end{tabular}


Table 3.4: Network prominence and director compensation

This table reports the coefficients associated with Prominence Pr-1 $_{1}$ in explaining director compensation. In columns (1), (3) and (6), the dependent variable is Paid $(0 / 1)_{t}$, a dummy variable that equals to 1 if a board of director receives zero compensation at year $t$ and 0 otherwise. In columns (2), (4) and (6), the dependent variable is $L n(\text { Compensation }+1)_{t}$, the logarithm of compensation plus 1 for a director in a firm at year t. Table 3.14 provides all variable definitions. The regressions control for year, industry, and number of directorships fixed effects. In parentheses are t-statistics based on standard errors adjusted for heteroskedasticity. Superscripts $*{ }^{* *}$ and ${ }^{* * *}$ denote significance at the $10 \%, 5 \%$ and $1 \%$ levels, respectively.

\begin{tabular}{|c|c|c|c|c|c|c|}
\hline \multirow[b]{3}{*}{ Explanatory variables } & \multirow{2}{*}{\multicolumn{2}{|c|}{ Independent director }} & \multicolumn{4}{|c|}{ Non-independent director } \\
\hline & & & \multicolumn{2}{|c|}{ Executive director } & \multicolumn{2}{|c|}{ Non-executive director } \\
\hline & $\begin{array}{c}\operatorname{Paid}(0 / 1)_{t} \\
(1)\end{array}$ & $\begin{array}{c}\operatorname{Ln}(\text { Compensation }+1)_{t} \\
(2)\end{array}$ & $\begin{array}{l}\operatorname{Paid}(0 / 1)_{t} \\
(3)\end{array}$ & $\begin{array}{c}\operatorname{Ln}(\text { Compensation }+1)_{t} \\
(4)\end{array}$ & $\begin{array}{l}\operatorname{Paid}(0 / 1)_{t} \\
\quad(5)\end{array}$ & $\begin{array}{c}\operatorname{Ln}(\text { Compensation }+1)_{t} \\
(6)\end{array}$ \\
\hline \multirow[t]{2}{*}{ Prominence $_{t-1}$} & $0.729^{* * *}$ & $13.088^{* * *}$ & -0.331 & $11.088^{* * *}$ & -0.381 & -4.631 \\
\hline & $(5.87)$ & $(9.13)$ & $(-1.11)$ & $(2.73)$ & $(-1.05)$ & $(-1.07)$ \\
\hline \multirow[t]{2}{*}{$\operatorname{Woman}(0 / 1)$} & -0.003 & -0.051 & $0.008^{* * *}$ & $0.057^{*}$ & $0.018^{* *}$ & $0.158^{*}$ \\
\hline & $(-1.06)$ & $(-1.56)$ & $(3.63)$ & $(1.87)$ & $(2.51)$ & $(1.92)$ \\
\hline \multirow[t]{2}{*}{$\mathrm{Aget}_{\mathrm{t}}$} & $0.003^{* *}$ & $0.045^{* * *}$ & 0.001 & $0.047^{* * *}$ & $-0.012^{* * *}$ & $-0.111^{* * *}$ \\
\hline & $(2.40)$ & $(3.57)$ & $(0.38)$ & $(2.59)$ & $(-5.21)$ & $(-4.07)$ \\
\hline \multirow{2}{*}{$A g e_{t}^{2}$} & $-0 * * *$ & $-0 * * *$ & -0 & $-0.001 * * *$ & $0 * * *$ & $0.001^{* * *}$ \\
\hline & $(-2.98)$ & $(-4.01)$ & $(-0.79)$ & $(-2.58)$ & $(5.21)$ & $(4.07)$ \\
\hline \multirow[t]{2}{*}{ Tenure $_{t}$} & $0.005^{* * *}$ & $0.050 * * *$ & $0.001^{* * *}$ & $0.018^{* * *}$ & $0.010^{* * *}$ & $0.126^{* * *}$ \\
\hline & $(8.50)$ & $(7.10)$ & $(3.06)$ & $(4.55)$ & $(13.98)$ & $(15.33)$ \\
\hline Busy director $(0 / 1)_{t}$ & $\begin{array}{l}-0.005 \\
(-0.48)\end{array}$ & $\begin{array}{l}-0.066 \\
(-0.53)\end{array}$ & & & & \\
\hline \multirow[t]{2}{*}{$C E O / C O B(0 / 1)_{t}$} & & & $-0.007^{* * *}$ & $0.193^{* * *}$ & $0.217^{* * *}$ & $3.236^{* * *}$ \\
\hline & & & $(-3.45)$ & $(7.28)$ & $(41.79)$ & $(49.57)$ \\
\hline \multirow[t]{2}{*}{ Related director $(0 / 1)_{t}$} & & & $-0.022^{* * *}$ & $-0.216^{* * *}$ & $-0.246 * * *$ & $-3.051 * * *$ \\
\hline & & & $(-8.41)$ & $(-6.29)$ & $(-55.26)$ & $(-57.75)$ \\
\hline \multirow[t]{2}{*}{ Ln $(\text { Share ownership }+1)_{t}$} & & & $0.001^{* * *}$ & $0.016^{* * *}$ & $0.011^{* * *}$ & $0.156^{* * *}$ \\
\hline & & & $(6.74)$ & $(10.10)$ & $(28.59)$ & $(33.40)$ \\
\hline \multirow[t]{2}{*}{ Ln (Board size $)_{t}$} & $0.022^{* * *}$ & $0.311^{* * *}$ & -0.003 & -0.105 & -0.008 & -0.058 \\
\hline & $(3.49)$ & $(4.48)$ & $(-0.49)$ & $(-1.38)$ & $(-0.63)$ & $(-0.39)$ \\
\hline \multirow{2}{*}{ Dualityt } & -0.004 & -0.040 & $-0.012^{* * *}$ & $-0.127 * * *$ & 0.009 & 0.104 \\
\hline & $(-1.39)$ & $(-1.32)$ & $(-5.45)$ & $(-4.38)$ & $(1.35)$ & $(1.37)$ \\
\hline \multirow[t]{2}{*}{ Independent director $(\%)_{t}$} & $0.110^{* * *}$ & $1.794^{* * *}$ & $-0.039^{* *}$ & $-0.534^{* *}$ & $0.101^{* *}$ & $1.399 * *$ \\
\hline & $(5.88)$ & $(8.56)$ & $(-2.08)$ & $(-2.13)$ & $(2.13)$ & $(2.45)$ \\
\hline \multirow{2}{*}{ Meeting frequency $(\text { Firm })_{t}$} & 0 & 0.001 & -0 & 0.006 & $0.004^{* * *}$ & $0.043^{* * *}$ \\
\hline & $(0.14)$ & $(0.43)$ & $(-0.51)$ & $(1.51)$ & $(6.30)$ & $(6.10)$ \\
\hline \multirow[t]{2}{*}{$\operatorname{Ln}(C E O \text { compensation }+1)_{t}$} & $0.001^{* * *}$ & $0.022^{* * *}$ & $0.011^{* * *}$ & $0.192^{* * *}$ & $0.004 * * *$ & $0.049^{* * *}$ \\
\hline & $(5.53)$ & $(7.51)$ & $(15.70)$ & $(20.39)$ & $(7.98)$ & $(9.12)$ \\
\hline State-owned $(0 / 1)_{t}$ & $-0.007^{* * *}$ & $-0.176 * * *$ & $-0.007^{* * *}$ & $-0.125^{* * *}$ & $-0.125^{* * *}$ & $-1.573^{* * *}$ \\
\hline & $(-2.93)$ & $(-6.73)$ & $(-3.48)$ & $(-4.61)$ & $(-24.79)$ & $(-26.26)$ \\
\hline Largest shareholder $(\%)_{t}$ & $0.024^{* * *}$ & $0.199^{* * *}$ & $0.011^{*}$ & $-0.142^{*}$ & $-0.231 * * *$ & $-2.694 * * *$ \\
\hline & $(3.60)$ & $(2.66)$ & $(1.82)$ & $(-1.78)$ & $(-15.86)$ & $(-15.61)$ \\
\hline Ln(Total Assets $)_{t}$ & $-0.004 * * *$ & $0.099^{* * *}$ & $-0.003^{* * *}$ & $0.201^{* * *}$ & $0.005^{* *}$ & $0.194^{* * *}$ \\
\hline & $(-4.56)$ & $(9.03)$ & $(-3.01)$ & $(14.16)$ & $(2.57)$ & $(7.74)$ \\
\hline Book leverage $_{t}$ & 0.008 & -0.013 & $-0.011^{*}$ & $-0.326^{* * *}$ & -0.016 & $-0.311^{* *}$ \\
\hline & $(1.37)$ & $(-0.21)$ & $(-1.65)$ & $(-3.75)$ & $(-1.27)$ & $(-2.20)$ \\
\hline Cash holdings $_{t}$ & $0.008^{* *}$ & $0.167^{* * *}$ & $-0.025^{* * *}$ & -0.171 & $-0.048^{* *}$ & $-0.506^{*}$ \\
\hline & $(2.31)$ & $(3.10)$ & $(-6.19)$ & $(-1.57)$ & $(-2.09)$ & $(-1.89)$ \\
\hline$R O A_{t-1}$ & $0.073^{* * *}$ & $1.094^{* * *}$ & $0.051^{* *}$ & $2.948^{* * *}$ & $0.218^{* * *}$ & $3.143^{* * *}$ \\
\hline & $(3.59)$ & $(4.96)$ & $(2.01)$ & $(9.26)$ & $(5.61)$ & $(7.05)$ \\
\hline Stock volatilityt-1 & $0.008^{* * *}$ & $0.106^{* * *}$ & -0.011 & -0.115 & $-0.036^{* * *}$ & $-0.436 * * *$ \\
\hline & $(2.74)$ & $(3.06)$ & $(-1.52)$ & $(-1.25)$ & $(-3.89)$ & $(-4.02)$ \\
\hline Year effects & Yes & Yes & Yes & Yes & Yes & Yes \\
\hline Industry effects & Yes & Yes & Yes & Yes & Yes & Yes \\
\hline Number of directorships effects & Yes & Yes & Yes & Yes & Yes & Yes \\
\hline$R^{2}$ & 0.014 & 0.021 & 0.085 & 0.180 & 0.202 & 0.239 \\
\hline Observations & 49,442 & 49,442 & 26,498 & 26,498 & 47,753 & 47,753 \\
\hline
\end{tabular}


Table 3.5: Network prominence and director compensation (firm and director fixed effects)

This table reports the coefficients associated with Prominence $_{t-1}$ in explaining director compensation when firm or director fixed effects are included. In columns (1), (3) and (6), the dependent variable is $\operatorname{Paid}(0 / 1)_{t}$, a dummy variable that equals to 1 if a board of director receives zero compensation at year $\mathrm{t}$ and 0 otherwise. In columns (2), (4) and (6), the dependent variable is Ln(Compensation+1) $)_{t}$, the logarithm of compensation plus 1 for a director in a firm at year t. In panel A, the regressions control for firm, year, industry, and number of directorships fixed effects. In panel B, the regressions control for director, year, industry, and number of directorships fixed effects. This table shares the same control variables as those in Table 3.4. Table 3.14 provides all variable definitions. In parentheses are t-statistics based on standard errors adjusted for heteroskedasticity and firm or director clustering. Superscripts $*$, $* *$ and ${ }^{* * *}$ denote significance at the $10 \%, 5 \%$ and $1 \%$ levels, respectively.

\begin{tabular}{|c|c|c|c|c|c|c|}
\hline \multirow[b]{4}{*}{ Explanatory variables } & \multicolumn{2}{|c|}{ Independent director } & \multicolumn{4}{|c|}{ Non-independent director } \\
\hline & \multirow{3}{*}{\multicolumn{2}{|c|}{$\operatorname{Paid}(0 / 1)_{t} \operatorname{Ln}(\text { Compensation }+1)_{t}$}} & \multicolumn{2}{|c|}{ Executive director } & \multicolumn{2}{|c|}{ Non-executive director } \\
\hline & & & $\operatorname{aid}(0 / 1)$ & mpensati & $\operatorname{Paid}(0 / 1$ & npensation +1$)_{t}$ \\
\hline & & & $(3)$ & (4) & $(5)$ & $(6)$ \\
\hline \multicolumn{7}{|l|}{ Panel A. Firm fixed effects } \\
\hline \multirow[t]{2}{*}{ Prominence $_{t-1}$} & $1.035^{* * *}$ & $14.036^{* * *}$ & -0.261 & 0.060 & 0.166 & 2.252 \\
\hline & $(6.21)$ & $(7.53)$ & $(-0.55)$ & $(0.01)$ & $(0.38)$ & $(0.42)$ \\
\hline Control variables & Yes & Yes & Yes & Yes & Yes & Yes \\
\hline Year effects & Yes & Yes & Yes & Yes & Yes & Yes \\
\hline Number of directorships effects & Yes & Yes & Yes & Yes & Yes & Yes \\
\hline Firm effects & Yes & Yes & Yes & Yes & Yes & Yes \\
\hline$R^{2}$ & 0.191 & 0.207 & 0.279 & 0.392 & 0.574 & 0.569 \\
\hline Observations & 49,442 & 49,442 & 26,498 & 26,498 & 47,753 & 47,753 \\
\hline \multicolumn{7}{|l|}{ Panel B. Director fixed effects } \\
\hline \multirow[t]{2}{*}{ Prominence $_{t-1}$} & $1.312^{* * *}$ & $18.757^{* * *}$ & -0.528 & -3.711 & $-0.712^{*}$ & -6.310 \\
\hline & $(5.77)$ & $(6.80)$ & $(-1.64)$ & $(-0.86)$ & $(-1.69)$ & $(-1.32)$ \\
\hline Control variables & Yes & Yes & Yes & Yes & Yes & Yes \\
\hline Year effects & Yes & Yes & Yes & Yes & Yes & Yes \\
\hline Number of directorships effects & Yes & Yes & Yes & Yes & Yes & Yes \\
\hline Industry effects & Yes & Yes & Yes & Yes & Yes & Yes \\
\hline Director effects & Yes & Yes & Yes & Yes & Yes & Yes \\
\hline$R^{2}$ & 0.317 & 0.335 & 0.675 & 0.725 & 0.875 & 0.884 \\
\hline Observations & 49,442 & 49,442 & 26,498 & 26,498 & 47,753 & 47,753 \\
\hline
\end{tabular}




\section{Table 3.6: Network prominence and director turnover}

This table reports the coefficients associated with Prominence $t$ in explaining director turnover. The dependent variable is Turnover $(0 / 1)_{t}$, a dummy variable that equals to 1 for a director in year $\mathrm{t}$ if he or she does not appear in the annual report in year $\mathrm{t}+1$ and 0 otherwise. Table 3.14 provides all variable definitions. The regressions control for year, industry, and number of directorships fixed effects. In parentheses are t-statistics based on standard errors adjusted for heteroskedasticity. Superscripts *, ** and $* * *$ denote significance at the $10 \%, 5 \%$ and $1 \%$ levels, respectively.

\section{Independent director Non-independent director} Executive director Non-executive director

Dependent variable $=$ Turnover $(0 / 1)_{t}$

\begin{tabular}{|c|c|c|c|}
\hline Explanatory variables & (1) & (2) & (3) \\
\hline Prominencet $_{t}$ & $\begin{array}{c}4.874^{* * *} \\
(16.93)\end{array}$ & $\begin{array}{c}5.756^{* * *} \\
(9.18)\end{array}$ & $\begin{array}{c}5.450^{* * *} \\
(14.13)\end{array}$ \\
\hline $\operatorname{Woman}(0 / 1)$ & $\begin{array}{c}-0.007^{*} \\
(-1.75)\end{array}$ & $\begin{array}{c}-0.021^{* * *} \\
(-4.21)\end{array}$ & $\begin{array}{l}0.004 \\
(0.85)\end{array}$ \\
\hline $\mathrm{Age}_{t}$ & $\begin{array}{c}-0.008^{* * *} \\
(-5.01)\end{array}$ & $\begin{array}{c}-0.007^{* * * *} \\
(-2.94)\end{array}$ & $\begin{array}{l}0.001 \\
(0.54)\end{array}$ \\
\hline$A g e_{t}^{2}$ & $\begin{array}{c}0 * * * \\
(5.05)\end{array}$ & $\begin{array}{c}0^{* * *} \\
(4.00)\end{array}$ & $\begin{array}{c}0 \\
(0.45)\end{array}$ \\
\hline Tenure $_{t}$ & $\begin{array}{c}0.064^{* * *} \\
(64.88)\end{array}$ & $\begin{array}{c}0.003^{* * *} \\
(6.14)\end{array}$ & $\begin{array}{c}0.008^{* * *} \\
(17.22)\end{array}$ \\
\hline Busy director $(0 / 1)_{t}$ & $\begin{array}{c}-0.258^{* * * *} \\
(-8.71)\end{array}$ & & \\
\hline $\operatorname{Ln}(\text { Compensation }+1)_{t}$ & $\begin{array}{c}-0.015^{* * *} \\
(-24.72)\end{array}$ & $\begin{array}{c}-0.010^{* * *} \\
(-9.02)\end{array}$ & $\begin{array}{c}-0.006 * * * \\
(-20.90)\end{array}$ \\
\hline$C E O / C O B(0 / 1)_{t}$ & & $\begin{array}{c}-0.076^{* * * *} \\
(-24.31)\end{array}$ & $\begin{array}{c}-0.097 * * * \\
(-29.14)\end{array}$ \\
\hline Related director $(0 / 1)_{t}$ & & $\begin{array}{c}-0.032^{* * * *} \\
(-9.41)\end{array}$ & $\begin{array}{c}-0.183^{* * *} \\
(-49.42)\end{array}$ \\
\hline Ln $(\text { Share ownership }+1)_{t}$ & & $\begin{array}{c}-0.001^{* * * *} \\
(-4.78)\end{array}$ & $\begin{array}{c}-0.002^{* * *} \\
(-8.13)\end{array}$ \\
\hline $\operatorname{Ln}(\text { Board size })_{t}$ & $\begin{array}{c}-0.088^{* * *} \\
(-9.62)\end{array}$ & $\begin{array}{c}-0.088^{* * * *} \\
(-7.88)\end{array}$ & $\begin{array}{c}-0.113^{* * *} \\
(-11.62)\end{array}$ \\
\hline Duality $_{t}$ & $\begin{array}{l}0.002 \\
(0.50)\end{array}$ & $\begin{array}{c}-0.023^{* * * *} \\
(-6.13)\end{array}$ & $\begin{array}{c}-0.016 * * * \\
(-3.20)\end{array}$ \\
\hline Independent director $(\%)_{t}$ & $\begin{array}{c}-0.175^{* * *} \\
(-6.00)\end{array}$ & $\begin{array}{l}0.067^{*} \\
(1.85)\end{array}$ & $\begin{array}{c}0.182^{* * *} \\
(5.00)\end{array}$ \\
\hline Meeting frequency $(\text { Firm })_{t}$ & $\begin{array}{c}0.003^{* * *} \\
(7.44)\end{array}$ & $\begin{array}{c}0.001^{* * *} \\
(2.60)\end{array}$ & $\begin{array}{c}0.002^{* * *} \\
(4.64)\end{array}$ \\
\hline State-owned $(0 / 1)_{t}$ & $\begin{array}{c}-0.022^{* * *} \\
(-6.20)\end{array}$ & $\begin{array}{l}0.005 \\
(1.24)\end{array}$ & $\begin{array}{c}0.028^{* * *} \\
(7.56)\end{array}$ \\
\hline Largest shareholder $(\%)_{t}$ & $\begin{array}{c}0.025^{* *} \\
(2.43)\end{array}$ & $\begin{array}{l}0.006 \\
(0.56)\end{array}$ & $\begin{array}{l}-0.007 \\
(-0.68)\end{array}$ \\
\hline $\operatorname{Ln}(\text { Total Assets })_{t}$ & $\begin{array}{c}-0.009 * * * \\
(-5.86)\end{array}$ & $\begin{array}{c}-0.010^{* * * *} \\
(-5.71)\end{array}$ & $\begin{array}{c}-0.009 * * * \\
(-5.90)\end{array}$ \\
\hline Book leverage $_{t}$ & $\begin{array}{l}-0.009 \\
(-1.16)\end{array}$ & $\begin{array}{c}0.033^{* * *} \\
(3.25)\end{array}$ & $\begin{array}{c}0.027^{* * *} \\
(3.15)\end{array}$ \\
\hline$R O A_{t}$ & $\begin{array}{l}-0.002 \\
(-0.08)\end{array}$ & $\begin{array}{c}-0.080^{* *} \\
(-2.17)\end{array}$ & $\begin{array}{c}-0.120^{* * *} \\
(-3.76)\end{array}$ \\
\hline Year effects & Yes & Yes & Yes \\
\hline Industry effects & Yes & Yes & Yes \\
\hline Number of directorships effects & Yes & Yes & Yes \\
\hline$R^{2}$ & 0.158 & 0.062 & 0.105 \\
\hline Observations & 45,417 & 24,819 & 45,831 \\
\hline
\end{tabular}


Table 3.7: Network prominence and director turnover (firm and director fixed effects)

This table reports the coefficients associated with Prominence $_{t}$ in explaining director turnover when firm or director fixed effects are included. The dependent variable is Turnover $(0 / 1)_{t}$, a dummy variable that equals to 1 for a director in year $\mathrm{t}$ if he or she does not appear in the annual report in year $\mathrm{t}+$ 1 and 0 otherwise. In Panel A, the regressions control for firm, year, industry, and number of directorships fixed effects. In Panel B, the regressions control for director, year, industry, and number of directorships fixed effects. This table shares the same control variables as those in Table 3.6. Table 3.14 provides all variable definitions. In parentheses are t-statistics based on standard errors adjusted for heteroskedasticity and firm or director clustering. Superscripts *, ** and ${ }^{* * *}$ denote significance at the $10 \%, 5 \%$ and $1 \%$ levels, respectively.

\section{Independent director Non-independent director}

Dependent variable=Turnover $(0 / 1)_{t}$

Executive director Non-executive director

Explanatory variables

(2)

(3)

Panel A. Firm fixed effects

Prominencet

$5.572^{* * *}$

(12.79)

$.174^{* * *}$

$6.262^{* *}$

Control variables

Year effects

Number of directorships effects

Firm effects

$R^{2}$

Yes

(7.77)

(7.78)

Observations

Yes

Yes

Yes

0.235

Yes
Yes

Yes

45,417

Yes

Yes

0.190

24,819

Yes
Yes

0.189

Panel B. Director fixed effects

\begin{tabular}{lccc}
\hline Prominence $_{t}$ & $7.136^{* * *}$ & $5.848^{* * *}$ & $8.207^{* * *}$ \\
Control variables & $(10.35)$ & $(5.91)$ & $(9.07)$ \\
Year effects & Yes & Yes & Yes \\
Number of directorships effects & Yes & Yes & Yes \\
Industry effects & Yes & Yes & Yes \\
Director effects & Yes & Yes & Yes \\
$R^{2}$ & Yes & Yes & Yes \\
Observations & 0.411 & 0.585 & 0.576 \\
\hline
\end{tabular}


Table 3.8: Network prominence and directors' future directorship

This table reports the coefficients associated with Prominence P $_{t}$ in explaining board of directors' ability to gain future directorships. The dependent variable is Directorship $p_{t+1}$, the number of directorships a director gains at year $t+1$. Table 3.14 provides all variable definitions. The regressions control for year fixed effects. In parentheses are t-statistics based on standard errors adjusted for heteroskedasticity. Superscripts $*{ }^{* *}$ and $* * *$ denote significance at the $10 \%, 5 \%$ and $1 \%$ levels, respectively.

\section{Independent director Non-independent director}

Executive director Non-executive director

Dependent variable $=$ Directorship D $_{t+1}$

Explanatory variables

\begin{tabular}{|c|c|c|c|}
\hline Prominence $_{t}$ & $\begin{array}{c}20.283^{* * *} \\
(16.10)\end{array}$ & $\begin{array}{c}5.678^{* * *} \\
(7.31)\end{array}$ & $\begin{array}{c}33.858^{* * *} \\
(21.89)\end{array}$ \\
\hline $\operatorname{Woman}(0 / 1)$ & $\begin{array}{c}-0.027^{* * *} \\
(-2.64)\end{array}$ & $\begin{array}{l}0.005 \\
(1.12)\end{array}$ & $\begin{array}{c}-0.023^{* * *} \\
(-3.95)\end{array}$ \\
\hline$A g e_{t}$ & $\begin{array}{c}0.032^{* * *} \\
(9.75)\end{array}$ & $\begin{array}{l}-0.002 \\
(-1.17)\end{array}$ & $\begin{array}{l}-0.003 \\
(-1.46)\end{array}$ \\
\hline$A g e_{t}^{2}$ & $\begin{array}{c}-0^{* * *} \\
(-11.18)\end{array}$ & $\begin{array}{c}0 \\
(1.17)\end{array}$ & $\begin{array}{c}0 \\
(1.41)\end{array}$ \\
\hline Tenure $_{t}$ & $\begin{array}{c}-0.016^{* * *} \\
(-7.91)\end{array}$ & $\begin{array}{c}0.002^{* * *} \\
(5.66)\end{array}$ & $\begin{array}{l}0.001^{*} \\
(1.94)\end{array}$ \\
\hline Busy director $(0 / 1)_{t}$ & $\begin{array}{c}1.956^{* * *} \\
(91.22)\end{array}$ & & \\
\hline $\operatorname{Ln}(\text { Compensation }+1)_{t}$ & $\begin{array}{c}0.031^{* * *} \\
(24.58)\end{array}$ & $\begin{array}{c}-0 \\
(-0.48)\end{array}$ & $\begin{array}{c}0 \\
(0.40)\end{array}$ \\
\hline$C E O / C O B(0 / 1)_{t}$ & & $\begin{array}{l}0.003 \\
(1.26)\end{array}$ & $\begin{array}{c}-0.016^{* * *} \\
(-3.73)\end{array}$ \\
\hline Related director $(0 / 1)_{t}$ & & $\begin{array}{c}-0.007^{* * *} \\
(-2.99)\end{array}$ & $\begin{array}{c}0.037^{* * *} \\
(8.00)\end{array}$ \\
\hline Ln $(\text { Share ownership }+1)_{t}$ & & $\begin{array}{c}-0.001^{* * *} \\
(-4.76)\end{array}$ & $\begin{array}{c}-0.003^{* * *} \\
(-10.21)\end{array}$ \\
\hline $\operatorname{Ln}(\text { Board size })_{t}$ & $\begin{array}{c}-0.312^{* * *} \\
(-13.97)\end{array}$ & $\begin{array}{c}-0.044^{* * *} \\
(-5.46)\end{array}$ & $\begin{array}{c}-0.414^{* * *} \\
(-21.82)\end{array}$ \\
\hline Duality $_{t}$ & $\begin{array}{l}0.011 \\
(1.23)\end{array}$ & $\begin{array}{c}0.010^{* * *} \\
(3.59)\end{array}$ & $\begin{array}{c}0.019 * * * \\
(3.08)\end{array}$ \\
\hline Independent director $(\%)_{t}$ & $\begin{array}{c}-0.272^{* * *} \\
(-3.99)\end{array}$ & $\begin{array}{c}-0.054^{* * *} \\
(-2.60)\end{array}$ & $\begin{array}{c}-0.376^{* * *} \\
(-8.97)\end{array}$ \\
\hline Meeting frequency $(\text { Firm })_{t}$ & $\begin{array}{c}-0.003^{* * *} \\
(-2.81)\end{array}$ & $\begin{array}{c}0.001^{*} \\
(1.70)\end{array}$ & $\begin{array}{l}-0.001 \\
(-1.34)\end{array}$ \\
\hline State-owned $(0 / 1)_{t}$ & $\begin{array}{c}0.028^{* * *} \\
(3.46)\end{array}$ & $\begin{array}{c}-0.008^{* * *} \\
(-3.02)\end{array}$ & $\begin{array}{c}0.010 * * \\
(2.11)\end{array}$ \\
\hline Largest shareholder $(\%)_{t}$ & $\begin{array}{c}0.101^{* * *} \\
(4.30)\end{array}$ & $\begin{array}{l}-0.001 \\
(-0.19)\end{array}$ & $\begin{array}{c}-0.025^{*} \\
(-1.90)\end{array}$ \\
\hline Ln(Total Assets $)_{t}$ & $\begin{array}{c}-0.015^{* * *} \\
(-4.82)\end{array}$ & $\begin{array}{c}0 \\
(0.34)\end{array}$ & $\begin{array}{l}-0.003 \\
(-1.58)\end{array}$ \\
\hline Book leverage $_{t}$ & $\begin{array}{c}0.057^{* * *} \\
(3.36)\end{array}$ & $\begin{array}{l}-0.004 \\
(-0.69)\end{array}$ & $\begin{array}{l}-0.011 \\
(-1.26)\end{array}$ \\
\hline$R O A_{t}$ & $\begin{array}{c}0.238^{* * *} \\
(3.91)\end{array}$ & $\begin{array}{c}0.063^{* * *} \\
(3.35)\end{array}$ & $\begin{array}{c}0.198^{* * *} \\
(6.14)\end{array}$ \\
\hline Year effects & Yes & Yes & Yes' \\
\hline$R^{2}$ & 0.576 & 0.017 & 0.188 \\
\hline Observations & 29,779 & 22,227 & 37,014 \\
\hline
\end{tabular}


Table 3.9: Network prominence and directors' future directorship (director fixed effects)

This table reports the coefficients associated with Prominence $t_{t}$ in explaining directors' ability to gain future directorships when director fixed effects are included. The dependent variable is Directorship $p_{t+1}$, the number of directorships a director gains at year $\mathrm{t}+1$. Table 3.14 provides all variable definitions. The regressions control for year and director fixed effects. This table shares the same control variables as those in Table 3.8. In parentheses are t-statistics based on standard errors adjusted for heteroskedasticity and director clustering. Superscripts $*, * *$ and $* * *$ denote significance at the $10 \%, 5 \%$ and $1 \%$ levels, respectively.

Independent director Non-independent director

Executive director Non-executive director

Dependent variable $=$ Directorship ${ }_{t+1}$

Explanatory variables

$(1)$

Prominence $_{t}$

Control variables

Year effects

Director effects

$R^{2}$

Observations
(2)

\begin{tabular}{ccc}
$3.899^{* * *}$ & $1.680^{* *}$ & $4.645^{* * *}$ \\
$(3.33)$ & $(2.51)$ & $(5.31)$ \\
Yes & Yes & Yes \\
Yes & Yes & Yes \\
Yes & Yes & Yes \\
0.820 & 0.733 & 0.839 \\
29,779 & 22,227 & 37,014 \\
\hline
\end{tabular}


Table 3.10: Indirect effect of network prominence on total compensation through turnover

This table reports the coefficients associated with the interaction term between Prominencet-1 and Turnover $(\%)_{t-1}$ in explaining aggregated director compensation. The dependent variable is $\operatorname{Ln}(\text { Total compensation }+1)_{t}$, the logarithm of 1 plus the aggregated compensation that a director collects from all firms at year t. Table 3.14 provides all variable definitions. The regressions control for year and director fixed effects. In parentheses are t-statistics based on standard errors adjusted for heteroskedasticity and director clustering. Superscripts *,** and *** denote significance at the $10 \%, 5 \%$ and $1 \%$ levels, respectively.

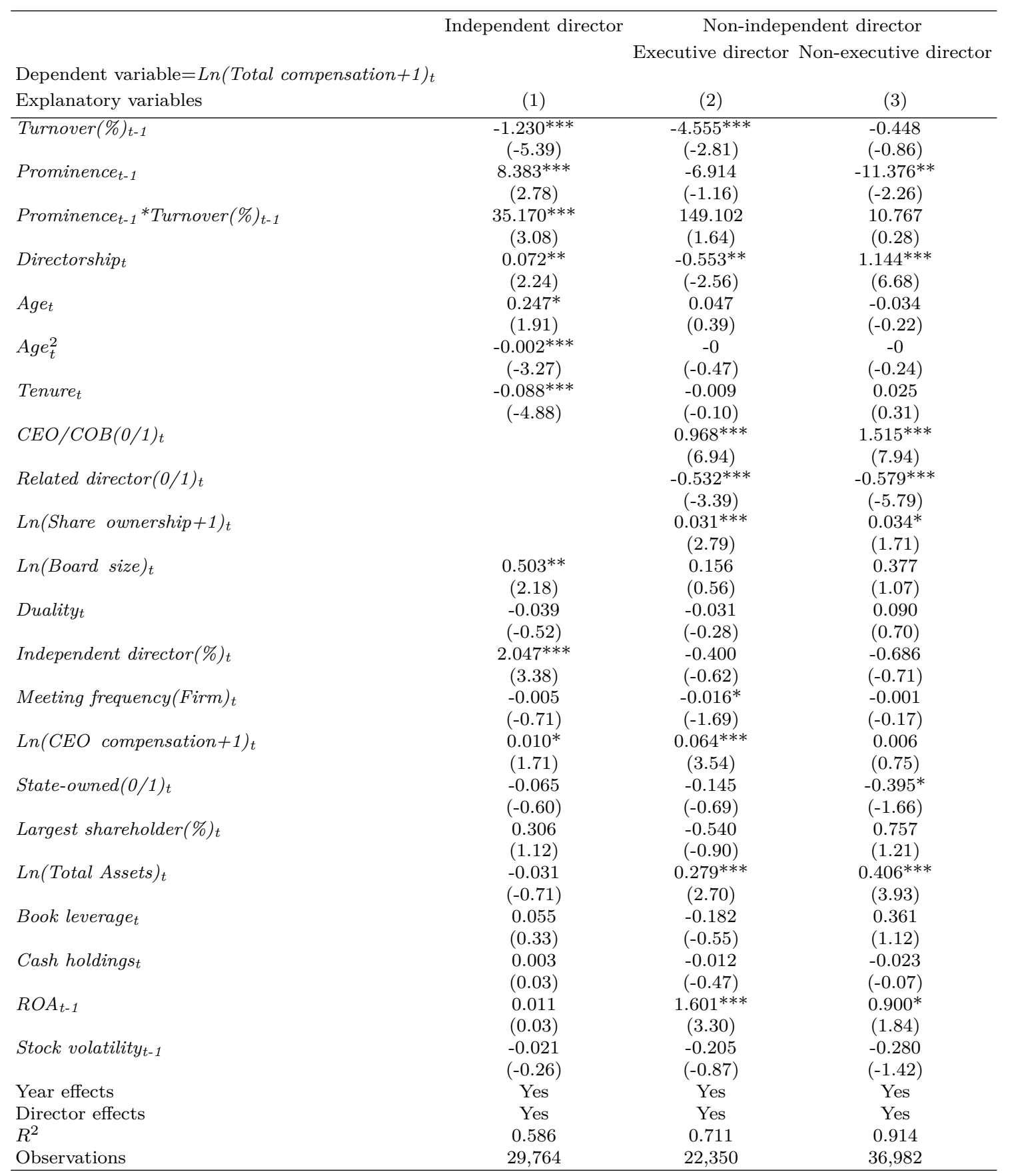


Table 3.11: Indirect effect of network prominence through related director on non-executive director compensation

This table reports the coefficients associated with the interaction term between Prominence $t_{-1}$ and Related director $(0 / 1)_{t}$ in explaining non-independent director compensation. In columns (1) and (3), the dependent variable is Paid(0/1) , a dummy variable that equals to 1 if a director receives zero compensation at year $\mathrm{t}$ and 0 otherwise. In columns (2) and (4), the dependent variable is $L n(\text { Compensation }+1)_{t}$, the logarithm of compensation plus 1 for a director at year t. In Panel A, the regressions control for firm, year, industry, and number of directorships fixed effects. In Panel B, the regressions control for director, year, industry, and number of directorships fixed effects. This table shares the same control variables as those in Table 3.4. Table 3.14 provides all variable definitions. In parentheses are t-statistics based on standard errors adjusted for heteroskedasticity and firm or director clustering. Superscripts *, ** and $* * *$ denote significance at the $10 \%, 5 \%$ and $1 \%$ levels, respectively.

Executive director $\quad$ Non-executive director

Explanatory variables

Paid(0/1) $)_{t}$ Ln(Compensation+1) $t_{t}$ Paid(0/1) Ln $_{t}$ Compensation+1) $)_{t}$

Panel A. Firm fixed effects

Prominence $e_{t-1}$

$(1)$

(3)

(4)

Related director $(0 / 1)_{t}$

0.275

$(0.64)$

$-0.022^{* * *}$

Prominence $_{t-1}{ }^{*}$ Related director $(0 / 1)_{t} \quad-2.725^{* *}$

$(-2.41)$

Control variables

Year effects

Number of directorships effects

Firm effects

$R^{2}$

Yes

Yes

Yes

Yes

0.280

26,498

8.777
$(1.55)$
$-0.237^{* * *}$
$(-2.79)$
$-44.329^{* * *}$
$(-2.83)$
Yes
Yes
Yes
Yes
0.393
26,498

$-0.413$

$-0.413-3.080$

$(-0.42) \quad(-0.26)$

$-0.204^{* * *} \quad-2.571^{* * *}$

$(-20.22) \quad(-20.75)$

Observations

26,498

$\begin{array}{ll}0.786 & 7.238 \\ (0.72) & (0.54)\end{array}$

Panel B. Director fixed effects

\begin{tabular}{lcccc}
\hline Prominence $_{t-1}$ & -0.552 & -3.492 & -0.402 & -2.807 \\
& $(-1.63)$ & $(-0.78)$ & $(-0.46)$ & $(-0.27)$ \\
Related director $(0 / 1)_{t}$ & -0.012 & -0.141 & $-0.050^{* * *}$ & $-0.567^{* * *}$ \\
& $(-1.19)$ & $(-1.12)$ & $(-5.51)$ & $(-5.31)$ \\
Prominence $_{t-1}{ }^{*}$ Related director $(0 / 1)_{t}$ & 0.133 & -1.199 & -0.425 & -4.788 \\
& $(0.15)$ & $(-0.10)$ & $(-0.45)$ & $(-0.44)$ \\
Control variables & Yes & Yes & Yes & Yes \\
Year effects & Yes & Yes & Yes & Yes \\
Number of directorships effects & Yes & Yes & Yes & Yes \\
Industry effects & Yes & Yes & Yes & Yes \\
Director effects & Yes & Yes & Yes & Yes \\
$R^{2}$ & 0.675 & 0.725 & 0.875 & 0.884 \\
Observations & 26,498 & 26,498 & 47,753 & 47,753 \\
\hline
\end{tabular}


This table reports the coefficients associated with the interaction term between Prominence $t_{t}$ and Related director(0/1) $t$ in explaining non-independent director turnover. The dependent variable is Turnover $(0 / 1)_{t}$, a dummy variable that equals to 1 for a director in year $t$ if he or she does not appear in the annual report in year $t+1$ and 0 otherwise. In Panel A, the regressions control for firm, year, industry, and number of directorships fixed effects. In Panel $\mathrm{B}$, the regressions control for director, year, industry, and number of directorships fixed effects. This table shares the same control variables as those in Table 3.6. Table 3.14 provides all variable definitions. In parentheses are t-statistics based on standard errors adjusted for heteroskedasticity and firm or director clustering. Superscripts $* * *$ and $* * *$ denote significance at the $10 \%, 5 \%$ and $1 \%$ levels, respectively.

Non-independent director

Executive director Non-executive director

Dependent variable $=$ Turnover $(0 / 1)_{t}$

Explanatory variables

$(2)$

Panel A. Firm fixed effects

Prominence $_{t}$

$\begin{array}{cc}7.575^{* * *} & 13.081^{* * *} \\ (7.54) & (8.93) \\ -0.033^{* * *} & -0.166^{* * *} \\ (-4.22) & (-20.53) \\ -2.329 & -9.591^{* * *} \\ (-1.52) & (-7.76) \\ \text { Yes } & \text { Yes } \\ \text { Yes } & \text { Yes } \\ \text { Yes } & \text { Yes } \\ \text { Yes } & \text { Yes } \\ 0.190 & 0.195 \\ 24,819 & 45,831\end{array}$

Observations

\begin{tabular}{lcc} 
& & \\
\hline Prominence $_{t}$ & $6.480^{* * *}$ & $13.423^{* * *}$ \\
Related director $(0 / 1)_{t}$ & $(5.89)$ & $(7.98)$ \\
& $-0.063^{* * *}$ & $-0.256^{* * *}$ \\
Prominence $_{t}{ }^{*}$ Related director $(0 / 1)_{t}$ & $(-4.30)$ & $(-19.40)$ \\
& $-3.668^{* *}$ & $-7.536^{* * *}$ \\
Control variables & $(-2.23)$ & $(-4.68)$ \\
Year effects & Yes & Yes \\
Number of directorships effects & Yes & Yes \\
Industry effects & Yes & Yes \\
Director effects & Yes & Yes \\
$R^{2}$ & Yes & Yes \\
Observations & 0.585 & 0.578 \\
\hline
\end{tabular}

Panel B. Director fixed effects 
Table 3.13: Indirect effect of network prominence through related director on non-independent directors' future directorships

This table reports the coefficients associated with the interaction term between Prominence $_{t}$ and Related director $(0 / 1)_{t}$ in explaining non-independent directors' ability to gain future directorships. The dependent variable is Directorship $p_{t+1}$, the number of directorships a director gains at year t+1. Table 3.14 provides all variable definitions. The regressions control for year and director fixed effects. This table shares the same control variables as those in Table 3.8 . In parentheses are t-statistics based on standard errors adjusted for heteroskedasticity and director clustering. Superscripts $*$, ** and $* * *$ denote significance at the $10 \%, 5 \%$ and $1 \%$ levels, respectively.

Non-independent director

Executive director Non-executive director

Dependent variable $=$ Directorship t $_{t+1}$

\begin{tabular}{lcc} 
Explanatory variables & $(1)$ & $(2)$ \\
\hline Prominence $_{t}$ & $1.894^{* *}$ & 0.158 \\
& $(2.53)$ & $(0.10)$ \\
${\text { Related director }(0 / 1)_{t}}$ & -0.004 & $0.018^{*}$ \\
Prominence $_{t}{ }^{*}$ Related director $(0 / 1)_{t}$ & $(-0.56)$ & $(1.71)$ \\
& -1.215 & $5.807^{* * *}$ \\
Control variables & $(-0.77)$ & $(3.29)$ \\
Year effects & Yes & Yes \\
Director effects & Yes & Yes \\
$R^{2}$ & Yes & Yes \\
Observations & 0.733 & 0.839 \\
\hline
\end{tabular}




\subsection{Appendix}

Table 3.14: Variable definitions

Variable Description

\section{Dependent variables}

$\operatorname{Paid}(0 / 1)_{t}$

Ln(Compensation +1$)_{t}$

Ln $(\text { Total compensation }+1)_{t}$

$\stackrel{N}{\Perp}$

Turnover $(0 / 1)_{t}$

Directorship $p_{t+1}$

\section{Variables of interest}

Prominence $_{t}$

Prominence $_{t-1}$

Turnover $(0 / 1)_{t-1}$

Turnover $(\%)_{t-1}$
The dummy variable equals to 1 if a board of director is paid in a firm in the year $t$ and 0 otherwise.

The logarithm of 1 plus the compensation that a board of director receives from a firm in the year $t$.

The logarithm of 1 plus the aggregated compensation that a board of director collects from all firms in the year $t$.

The measure of director turnover activity in the year $t$, which is a dummy variable equals to 1 for an observation in year $t$ if a board of director does not appear in the annual report in the year $t+1$ and 0 otherwise.

The number of directorships a board of director gains in the year $t+1$.

The eigenvector centrality of a board of director in the year $t$.

The eigenvector centrality of a board of director in the year $t-1$.

The measure of director turnover activity in the year $t-1$, which is a dummy variable equals to 1 for an observation in the year $t-1$ if a board of director does not appear in the annual report in the year $t$ and 0 otherwise.

The ratio of turnover in the year $t-1$, which equals to Turnover $(0 / 1)_{t-1}$ scaled by the number of directorships in the year $t-1$. 


\section{Variable}

\section{Description}

\section{Control variables}

$\operatorname{Woman}(0 / 1)$

Age $_{t}$

$A g e_{t}^{2}$

Tenure $_{t}$

Busy director $(0 / 1)_{t}$

농

Meeting frequency $(\text { Firm })_{t}$

Ln $(C E O \text { compensation }+1)_{t}$ $C E O / C O B(0 / 1)_{t}$

Related director $(0 / 1)_{t}$

Ln(Share ownership +1$)_{t}$

Ln(Board size $)_{t}$

Duality $_{t}$

Independent director $(\%)_{t}$

State-owned $(0 / 1)_{t}$
The dummy variable equals to 1 if a board of director is female and 0 otherwise.

The age of a board of director in the year $t$.

The square of age of a board of director in the year $t$.

The number of years that a board of director has served as a board of director in the year $t$.

The dummy variable equals to 1 if an independent director has more than two directorships in the year $t$ and 0 otherwise.

The number of board meetings for a firm in the year $t$.

The logarithm of 1 plus the compensation that a CEO receives from the firm in the year $t$.

The dummy variable equals to 1 if a board of director is CEO or COB in the year $t$ and 0 otherwise.

The dummy variable equals to 1 if a board of director holds a position in the controlling firm in the year $t$ and 0 otherwise.

The logarithm of a board of director's share holding plus 1 in the year $t$.

The logarithm of the number of directors on board in the year $t$.

The dummy variable equals to 1 if the CEO and chairman is the same person in the year $t$ and 0 otherwise.

The ratio of independent directors on board in the year $t$.

The dummy variable equals to 1 if the firm is state-owned in the year $t$ and 0 otherwise. 
Table 3.14: Variable definitions

Variable

Largest shareholder $(\%)_{t}$

Ln(Total Assets)

Book leverage ${ }_{t}$

Cash holdings $s_{t}$

$R O A_{t-1}$

Stock volatilityt-1

Ln (Compensation +1$)_{t}$

ㄴ.

\section{Description}

The percentage of share holding by the largest shareholders in the year $t$.

The logarithm of total assets in the year $t$.

The book value of total debts scaled by book value of total assets in the year $t$.

The cash and marketable security divided by the book value of total assets in the year $t$.

The net income scaled by the book value of total assets in the year $t-1$.

The variance of monthly stock returns in the year $t-1$.

The logarithm of 1 plus the compensation that a board of director receives from a firm in the year $t$.

The net income scaled by the book value of total assets in the year $t$. 